\title{
Uncertainty Quantification of Calculated Temperatures for the U.S. Capsules in the AGR-2 Experiment
}

The INL is a

U.S. Department of Energy

National Laboratory

operated by

Battelle Energy Alliance

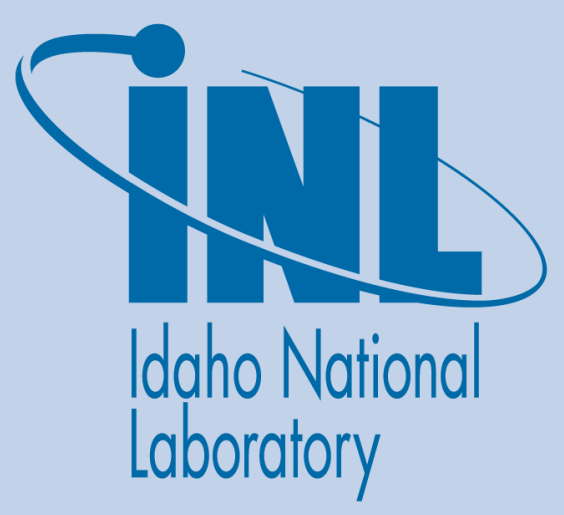

Binh T. Pham Jeffrey J. Einerson Grant L. Hawkes

March 2015

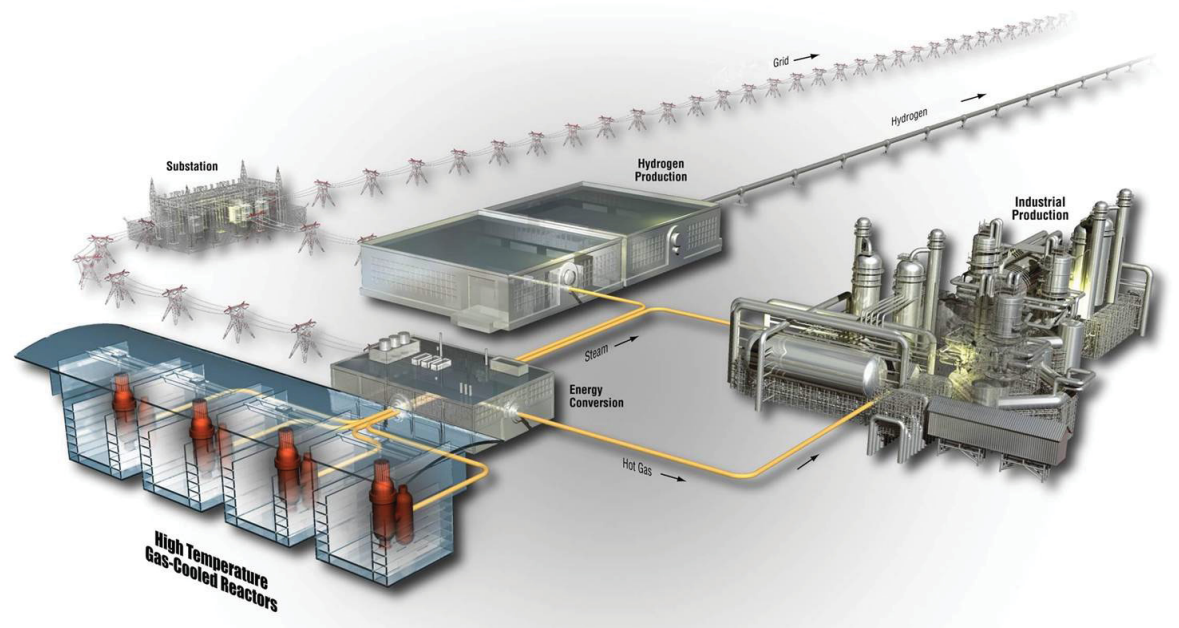




\section{DISCLAIMER}

This information was prepared as an account of work sponsored by an agency of the U.S. Government. Neither the U.S. Government nor any agency thereof, nor any of their employees, makes any warranty, expressed or implied, or assumes any legal liability or responsibility for the accuracy, completeness, or usefulness, of any information, apparatus, product, or process disclosed, or represents that its use would not infringe privately owned rights. References herein to any specific commercial product, process, or service by trade name, trade mark, manufacturer, or otherwise, does not necessarily constitute or imply its endorsement, recommendation, or favoring by the U.S. Government or any agency thereof. The views and opinions of authors expressed herein do not necessarily state or reflect those of the U.S. Government or any agency thereof. 


\title{
Uncertainty Quantification of Calculated Temperatures for the U.S. Capsules in the AGR-2 Experiment
}

\author{
Binh T. Pham \\ Jeffrey J. Einerson \\ Grant L. Hawkes
}

March 2015

\begin{abstract}
Idaho National Laboratory
INL ATR Program

Idaho Falls, Idaho 83415
\end{abstract}

http://www.inl.gov

Prepared for the

U.S. Department of Energy

Office of Nuclear Energy

Under DOE Idaho Operations Office

Contract DE-AC07-05ID14517 



\section{ART Program}

\section{Uncertainty Quantification of Calculated Temperatures for the U.S. Capsules in the AGR 2 Experiment}

INLEXT-15-34587

March 2015

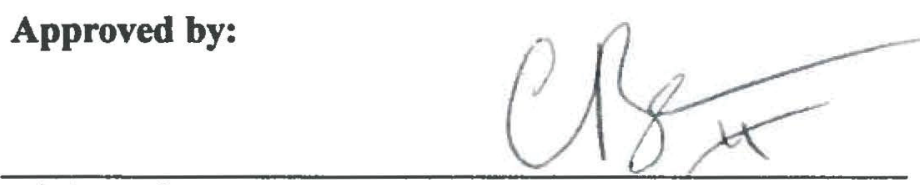

Binh T. Pham

Author
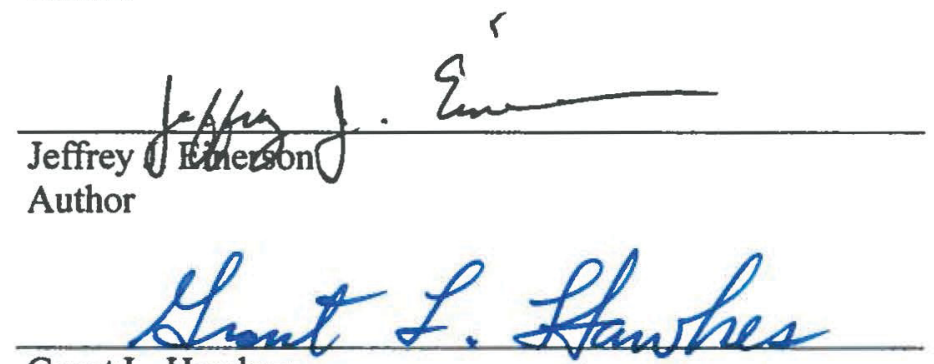

Grant L. Hawkes

Author

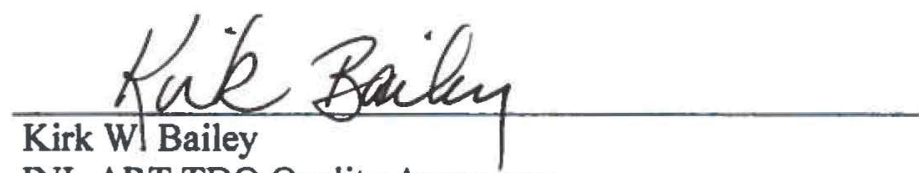

INL ART TDO Quality Assurance
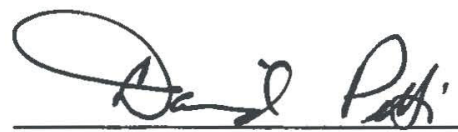

David A. Petti

ART Co-National Technical Director
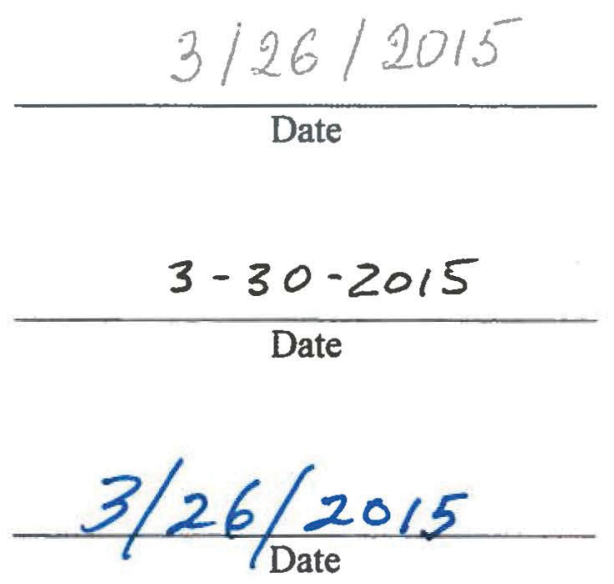

$\frac{3 \cdot 26 \cdot 2015}{\text { Date }}$

$3-30-2015$

Date 



\section{ABSTRACT}

This report documents the quantification of uncertainty of the calculated temperature data for the second Advanced Gas Reactor (AGR-2) fuel irradiation experiment conducted in the Advanced Test Reactor at Idaho National Laboratory in support of the Advanced Reactor Technology Research and Development program. Recognizing uncertainties inherent in physics and thermal simulations of the AGR-2 test, the results of the numerical simulations are used in combination with statistical analysis methods to improve qualification of measured data. The temperature simulation data for AGR tests are also used for validation of the fission product transport and fuel performance simulation models. These crucial roles of the calculated fuel temperatures in ensuring achievement of the AGR experimental program objectives require accurate determination of the model temperature uncertainties.

To quantify the uncertainty of AGR-2 calculated temperatures performed by the ABAQUS finite element heat transfer code, this study identifies and analyzes model parameters of potential importance to the AGR-2 predicted thermocouple and fuel temperatures. The selection of input parameters for uncertainty quantification is based on the ranking of their influences on variation of temperature predictions. Thus, selected input parameters include those with high sensitivity and those with the largest uncertainty. Propagation of model parameter uncertainty and sensitivity is then used to quantify the overall uncertainty of AGR-2 calculated temperatures. Expert judgment is used as the basis to specify the uncertainty range for selected input parameters. The input uncertainties are dynamic, accounting for the effect of unplanned events and changes in thermal properties of capsule components over extended exposure to high temperatures and fast neutron irradiation.

The sensitivity analysis performed in this work went beyond the traditional local sensitivity. Using experimental design, analysis of pairwise interactions of model parameters was performed to establish sufficiency of the first-order (linear) expansion terms in constructing the response surface. To achieve completeness, uncertainty propagation made use of pairwise noise correlations of model parameters. Furthermore, using an interpolation scheme over the input parameter domain, the analysis obtains time-dependent sensitivity over the test campaign duration. This allows computation of uncertainty for the predicted peak fuel temperatures and the predicted graphite temperatures at thermocouple locations during the entire AGR-2 irradiation period. 


\section{SUMMARY}

\section{S-1. Introduction}

This report documents the quantification of uncertainty in the calculated temperatures for the second Advanced Gas Reactor (AGR-2) fuel irradiation experiment. These experiments, conducted in the Advanced Test Reactor (ATR) at Idaho National Laboratory (INL), are in support of the Advanced Reactor Technology (ART) research and development (R\&D) program. While not possible to obtain by direct measurements in the tests, crucial fuel conditions (e.g., temperature, neutron fast fluence, and burnup) are calculated using core physics (JMOCUP) and thermal modeling (ABAQUS) codes. Calculated fuel temperatures serve crucial roles in achieving AGR experimental program objectives and require accurate determination of the model temperature uncertainties. One such role is the validation of the fission product transport and fuel performance simulation models.

\section{S-2. Approach}

To quantify the uncertainty of AGR calculated temperatures, ABAQUS code's finite element-based thermal model input parameters of potential importance are identified. Identification has two parts: (1) using expert judgment, determine parameters with the largest uncertainties and estimate these uncertainties, and (2) using sensitivity analysis, determine parameters that the modeling is most sensitive to. A set of parameters is selected for predicted temperature uncertainty quantification, including those with high sensitivity and/or those with large uncertainty. The parameter uncertainties and sensitivity coefficients are combined and propagated to quantify the overall uncertainty using Equation ( $S$-1), because the predicted temperature can be assumed to be the weighted summation of input parameters. This assumption is confirmed during sensitivity analysis.

$\sigma_{T}^{2}=\sum_{i}^{n} a_{i}^{2} \sigma_{i}^{2}+\sum_{i}^{n} \sum_{j \neq i}^{n} \rho_{i j} a_{i}^{2} \sigma_{i}^{2} a_{j}^{2} \sigma_{j}^{2}$

where

$\sigma_{T}^{2}$ is the overall temperature uncertainty in terms of variance

$a_{i}^{2}$ is the square of the sensitivity coefficient for parameter $i$

$\sigma_{i}^{2}$ is the uncertainty of input parameter $i$ in terms of variance

$\rho_{i j}$ is the correlation coefficient for input parameters $i$ and $j$.

The identified-as-important model parameters in terms of influence on uncertainty are: control gas gap width, heat rate in fuel compacts, neon fraction, graphite conductivity, and fuel conductivity. These are shown in Figure S-1. 


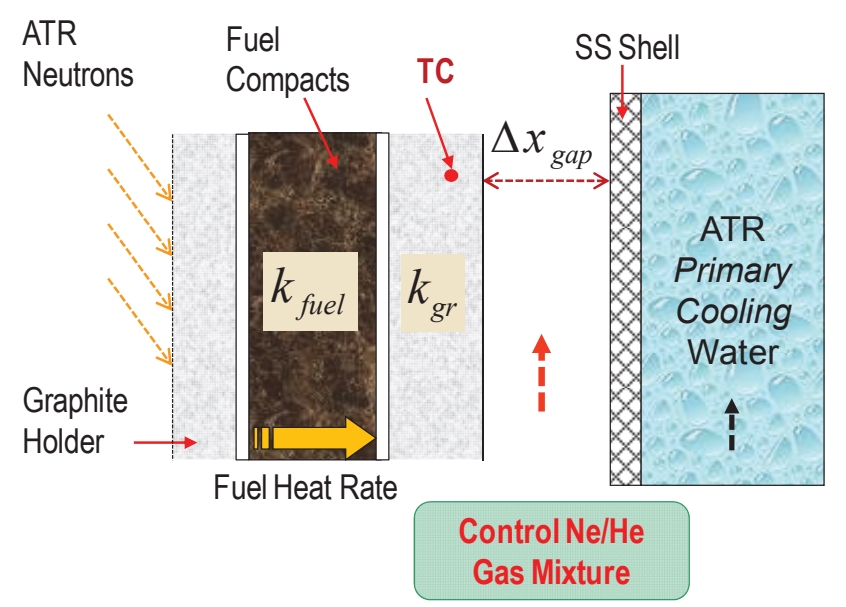

Figure S-1. Physical sketch of the axial cut of an AGR-2 Capsules.

Early analysis of thermocouple data indicated that they performed reliably during the beginning cycles of irradiation. Therefore, the thermal models for AGR-2 capsules were calibrated by varying the emissivity of surfaces of the graphite holder and stainless-steel retainer to best match temperatures at thermocouple (TC) locations with actual TC measurements during this portion of the irradiation. The residuals are the differences between measured and calculated temperatures for operational TCs. Continued monitoring and analysis of residuals beyond the calibration period show a pattern around and near zero for at least one TC in every capsule. This suggests negligible model bias and therefore the uncertainty quantification performed here relies solely on the model input parameters.

The graphite holders in six AGR-2 capsules have the same boron concentration as AGR-1 Capsule 6, so the holder shrinkage is assumed equal to the shrinkage rate seen in AGR-1 Capsule 6. This leads to the control gap increasing linearly with accumulated fast fluence irradiation. The excellent consistency in TC residual trend over the entire range of capsule thermal conditions throughout the AGR-2 irradiation provides confidence that the thermal model has appropriately included all important physical phenomena occurring in the capsule. This supports the assumption of negligible model bias and the decision to base the uncertainty analysis solely on model input parameters.

\section{S-3. Input Parameter Uncertainty}

The uncertainties of the input parameters of interest in the thermal model were estimated by ART R\&D program experts and are presented in Table S-1 along with the basis for the estimates. The input uncertainties of the control gap distance and neon fraction are dynamic, accounting for the effect of unplanned events (e.g., gas line cross-talk increases neon fraction uncertainty between $\sim 240$ effective full-power days (EFPD) (when the failure started during ATR Cycle 150B) and 280 EFPD (when the uniform gas mixtures were used) and changes in thermal properties of capsule components over extended exposure to high temperatures and fast neutron irradiation (e.g., assumed gap increase adds uncertainty in the gap distance) as shown in Figure S-2. 
Table S-1. Uncertainties of the most significant parameters of the AGR-2 thermal model.

\begin{tabular}{|l|c|l|}
\hline Parameter & Uncertainty (\%) & \multicolumn{1}{c|}{ Rationale } \\
\hline $\begin{array}{l}\text { Control gap } \\
\text { distance } \\
\text { neutron fluence in } \\
\text { following range: } \\
\text { Capsule 6: } 4.3-6.5 \\
\text { Capsule 5: } 6.5-9.7 \\
\text { Capsule 3:3.2-4.8 } \\
\text { Capsule 2: 3.7-5.5 }\end{array}$ & $\begin{array}{l}\text { Uncertainty at start of irradiation is 1 mil based on fabrication } \\
\text { tolerance. } \\
\text { Uncertainty over the course of irradiation is time dependent } \\
\text { because of AGR-2 graphite holder shrinkage. The graphite } \\
\text { shrinkage is proportional to the reaction rate in the graphite, } \\
\text { leading to a physics-based linear gas gap model similar to } \\
\text { AGR-1 Capsule 6, which has similar boron content. Thus, the } \\
\text { gap uncertainty is assumed to be a linear function of fluence } \\
\text { and is increased to 150\% of initial uncertainty by the end of } \\
\text { irradiation (Subsection 3.3.1). }\end{array}$ \\
\hline Neon fraction & Function of neon \\
fraction & $\begin{array}{l}\text { For the time periods before cross-talk and during uniform gas } \\
\text { mixture, uncertainty is based on 1 sccm flow rate tolerance. } \\
\text { For the time period between cross-talk start and uniform gas } \\
\text { mixture start (ATR Cycle 150B), uncertainty is estimated } \\
\text { from neon fraction prediction equation (Subsection 3.3.2). }\end{array}$ \\
\hline Fuel heat rate & 5 & $\begin{array}{l}\text { Basis is AGR-1 comparison done by J. Harp with additional } \\
\text { input from J. Sterbentz (Subsection 3.3.3). }\end{array}$ \\
\hline $\begin{array}{l}\text { Graphite } \\
\text { conductivity }\end{array}$ & \multirow{2}{*}{$\begin{array}{l}\text { Fuel } \\
\text { conductivity }\end{array}$} & $\begin{array}{l}\text { Additional conductivity data for the test graphite allows a } \\
\text { lower uncertainty estimate for graphite than for fuel. }\end{array}$ \\
\hline & $\begin{array}{l}\text { Uncertainty is based on work done on surrogate compacts by } \\
\text { C. Folsom at Utah State University. }\end{array}$ \\
\hline
\end{tabular}

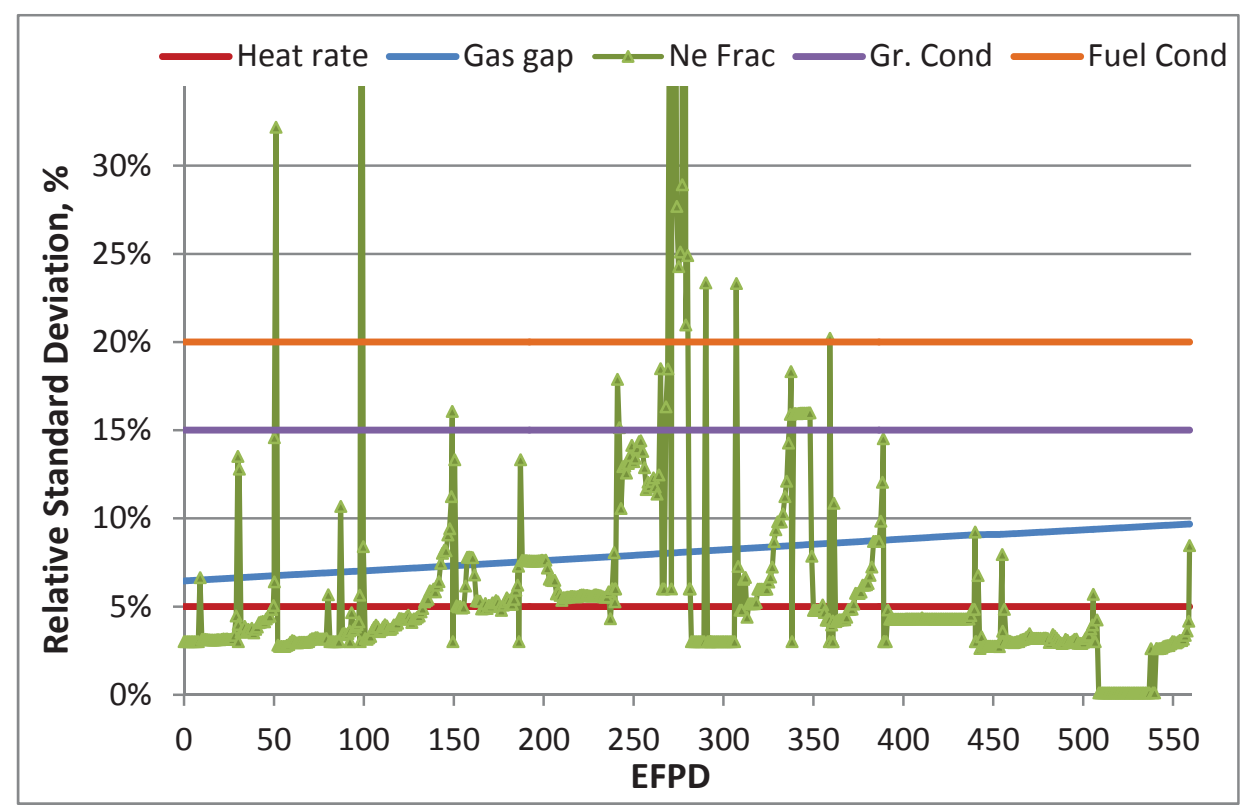

Figure S-2. Capsule 5 input parameter uncertainties in terms of relative standard deviation. 


\section{S-4. Input Parameter Sensitivity}

The parameter sensitivity analysis of the thermal model is performed to determine the sensitivity coefficients of the most influential variables. Capsule 5 data is used for the sensitivity analysis and is assumed to be representative of the other capsules. A sensitivity coefficient describes how the model predicted temperature would be influenced by changes in an input parameter. The overall uncertainty of the model output increases as the absolute sensitivity coefficient of an input parameter increases.

The sensitivity analysis performed here went beyond the traditional local sensitivity. Using experimental design, analysis of pairwise interactions of model parameters was also performed to establish sufficiency of the first-order (linear) expansion terms in the uncertainty propagation formula Equation (S-1). The sensitivity analysis was performed for four days throughout irradiation and an interpolation scheme over the input parameter domain was then used to obtain time-dependent sensitivity. This allows computation of uncertainty for the predicted fuel temperatures and the predicted graphite temperatures at TC locations over the whole AGR-2 irradiation period.

The parameter sensitivity coefficients for volume-average (VA) and peak fuel temperatures and TC temperature as function of (EFPD) are presented in Figure S-3 and the following conclusions are drawn:

- In general, the sensitivity coefficients of fuel fission heat rate (red lines) are highest (up to 0.58) followed by the neon fraction (green lines) and the control gas gap distance (blue lines) for all predicted temperatures of interest (VA fuel, peak fuel, and TC).

- For control gas gap distance, the sensitivity coefficients range from 0.2 to 0.4 . The sensitivity is decreasing over time because the control gas gap is increasing due to the graphite holder shrinkage under irradiation. Also, the peripheral TC is the most sensitive to the control gap distance uncertainty, followed by the VA fuel temperature and then by the peak fuel temperature.

- The neon fraction sensitivity coefficient is strongly dependent on neon fraction: it ranges from $\sim 0.1$ at a low neon fraction of 0.27 to more than 0.4 at a high neon fraction of 0.94 .

- The sensitivity coefficients of fuel compact thermal conductivity (orange lines) and graphite holder thermal conductivity (purple lines) are less than 0.1 for fuel temperatures. They are near zero for peripheral TC temperatures. 


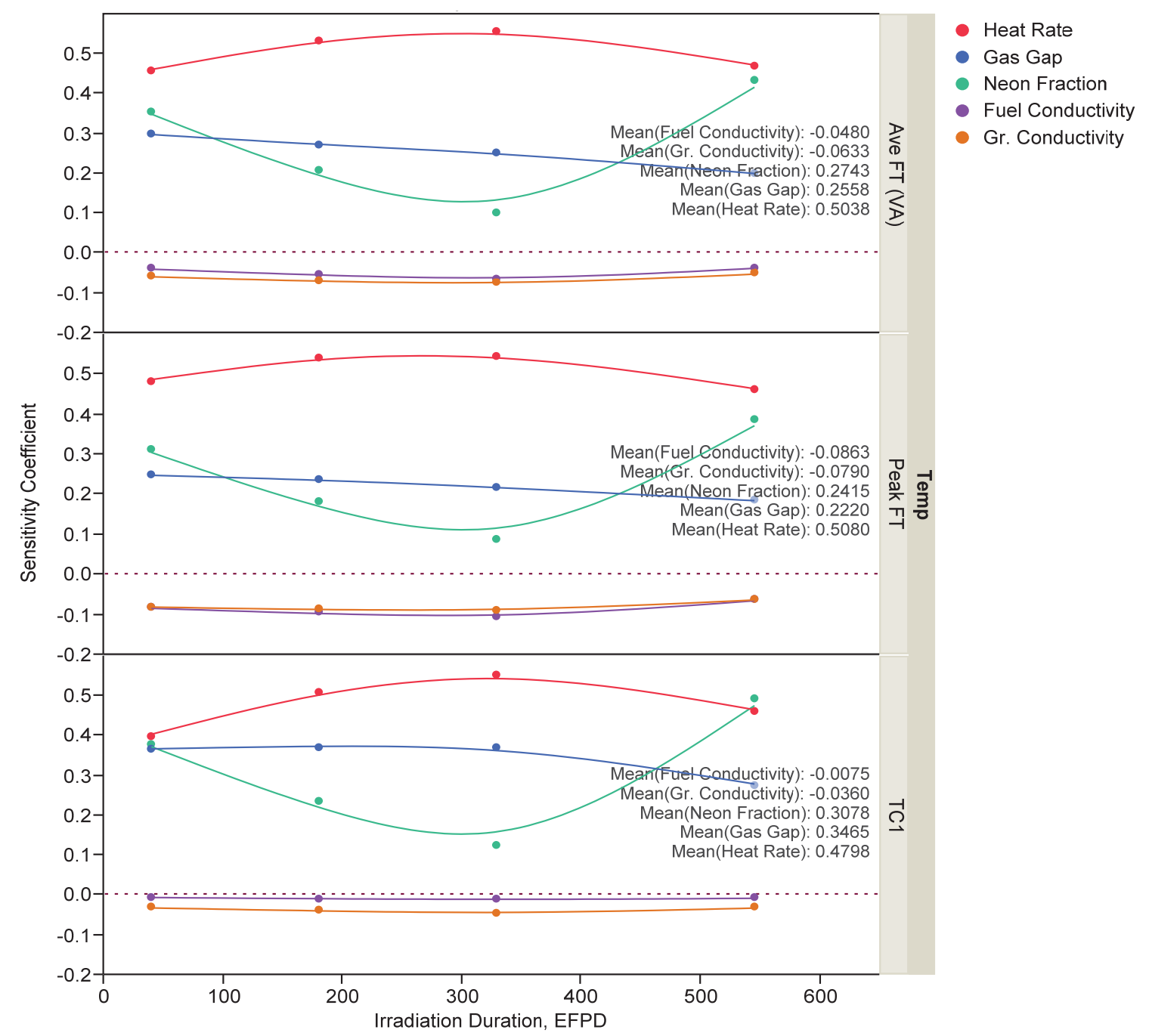

Figure S-3. Parameter sensitivity coefficients for fuel and thermocouple temperatures in Capsule 5.

\section{S-5. Combining Parameter Sensitivity and Uncertainty}

The overall uncertainty of a calculated temperature in terms of variance is obtained through propagation of model parameter uncertainty as the summation of the parameter variances weighted by the squares of their sensitivity coefficients Equation (S-1). Thus, the effect of a parameter on the model prediction variation is a product of input uncertainty and the sensitivity coefficient. In general, the $5 \%$ fuel heat rate uncertainty is the most significant factor contributing to overall uncertainty of the AGR-2 temperature predictions as shown in Figure S-4 for Capsule 5 (smallest control gas gap) and Figure S-5 for Capsule 2 (hottest fuel temperature). A large increase in variance is seen around 250 EFPD due to neon fraction uncertainty caused by gas line cross-talk. The following conclusions are drawn:

- The fuel heat rate is the most influential factor on overall uncertainty of VA fuel temperature (red crosses in the top plots) in both Capsules 2 and 5, with a maximum variance near the middle of irradiation when the fuel heat rate is highest. The higher gap uncertainty in Capsule 5 is the second most influential factor in VA fuel temperature uncertainty. 
- For peak fuel temperature, the most influential factor is also the fuel heat rate (red crosses in the middle plots), which has the largest sensitivity coefficient (up to 0.56). This is followed by the fuel thermal conductivity (orange line). The gas gap (blue line) in Capsule 5 becomes the second factor by the end of irradiation due to increasing gap uncertainty caused by fast neutrons.

- For TC temperature, the gas gap in Capsule 5 (blue line on bottom plot in Figure S-4) is the dominant factor because the smallest gap has highest uncertainty and sensitivity. In contrast, the fuel heat rate remains the dominant factor for TC temperature in Capsule 2 (Figure S-5). The fuel and graphite thermal conductivities have only minor impacts on TC temperature uncertainty.
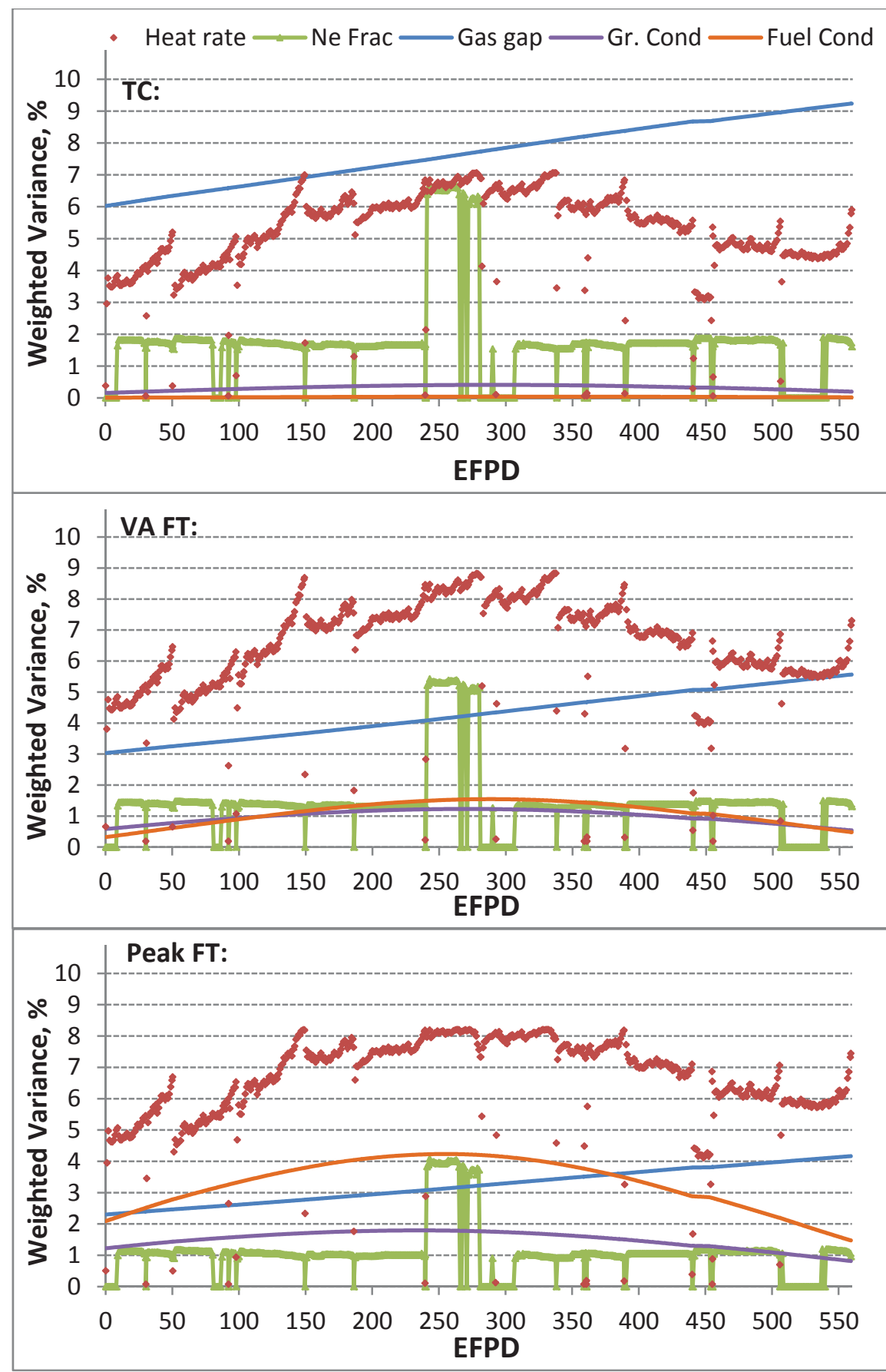

Figure S-4. Capsule 5 daily weighted temperature variances due to parameter uncertainties. 


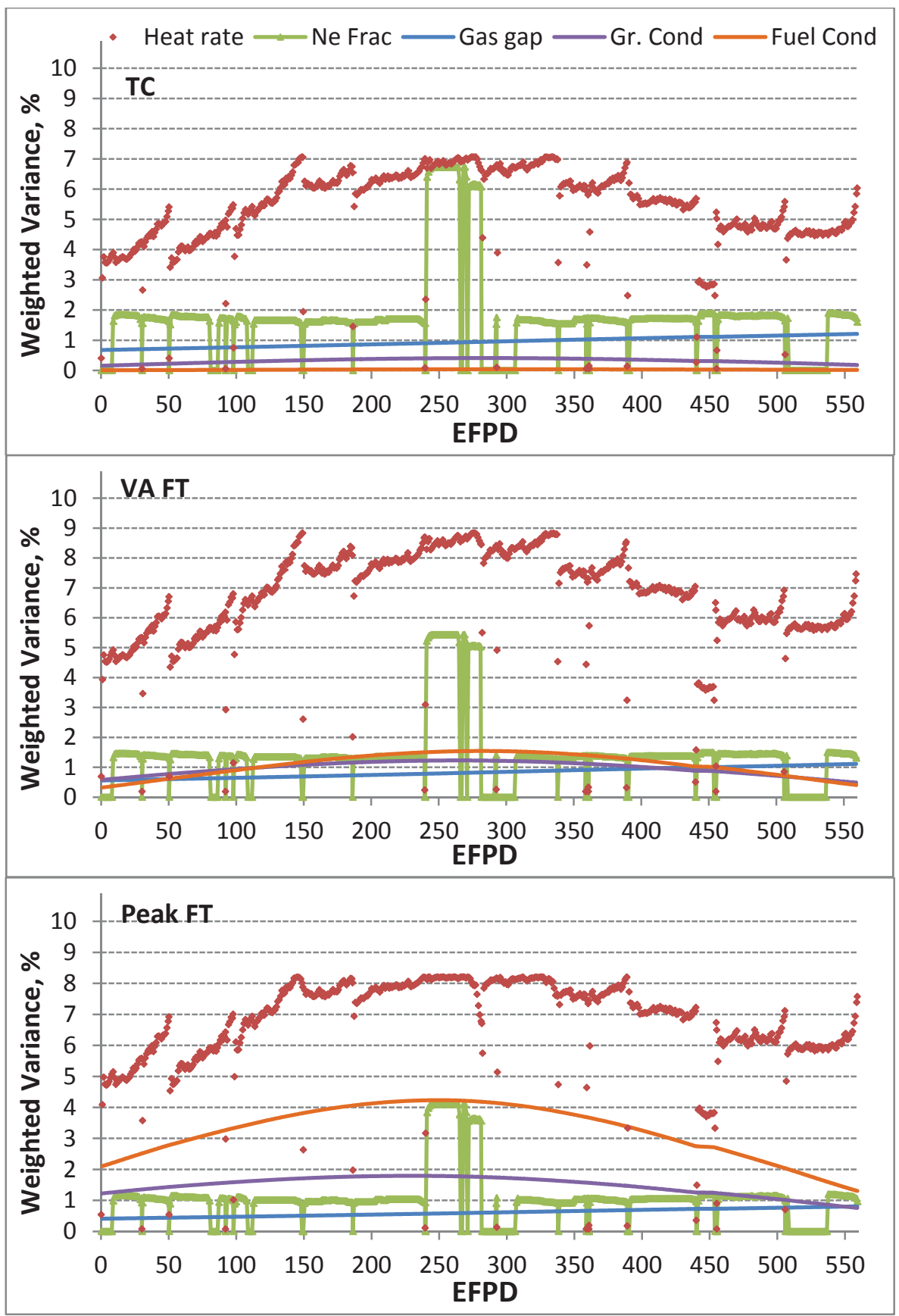

Figure S-5. Capsule 2 daily weighted temperature variances due to parameter uncertainties.

\section{S-6. Overall Uncertainty}

The overall uncertainty in the calculated temperatures for AGR-2 ranged from $2.1 \%$ to $4.0 \%$, depending on irradiation time (thermal conditions), capsule, and the temperature parameter being predicted (peak, volume-average, or TC). Table S-2 presents results of uncertainty quantification for instantaneous TC and fuel temperatures in four U.S. capsules. This table also includes overall uncertainty for time-average fuel temperatures at the end of irradiation. The dominant parameter is the input parameter with the largest influence on the overall uncertainty. The dominant sensitivity presents the range of the sensitivity coefficients for the dominant input parameter over all EFPDs. The dominant weighted variance is the range of the relative variances of calculated temperatures due to the 
dominant input parameter uncertainty. For instantaneous temperatures in each capsule, the uncertainty results represent maximum and minimum values during the entire irradiation. For time-average fuel temperatures, the results represent relative $(\%)$ and absolute $\left({ }^{\circ} \mathrm{C}\right)$ uncertainties at the end of irradiation (bottom rows in Table S-2).

Table S-2. Temperature uncertainty results for U.S. AGR-2 capsules.

\begin{tabular}{|c|c|c|c|c|c|c|}
\hline \multirow[b]{2}{*}{ Capsule } & & $\mathrm{TC}$ & \multicolumn{2}{|c|}{ VA FT } & \multicolumn{2}{|c|}{ Peak FT } \\
\hline & \multicolumn{6}{|c|}{ Instantaneous temperature } \\
\hline \multirow{5}{*}{$\begin{array}{c}6 \\
\text { (top - least } \\
\text { fast } \\
\text { fluence) }\end{array}$} & Dominant parameter & Fuel heat rate & \multicolumn{2}{|c|}{ Fuel heat rate } & \multicolumn{2}{|c|}{ Fuel heat rate } \\
\hline & Dominant sensitivity & $0.3-0.52$ & \multicolumn{2}{|c|}{$0.37-0.58$} & \multicolumn{2}{|c|}{$0.35-0.57$} \\
\hline & Dominant weighted variance, $\%$ & $2.6-6.7$ & \multicolumn{2}{|c|}{$3.0-8.3$} & \multicolumn{2}{|c|}{$3.0-8.1$} \\
\hline & Relative uncertainty, $\%$ & $2.3-3.6$ & \multicolumn{2}{|c|}{$2.6-3.6$} & \multicolumn{2}{|c|}{$2.8-3.9$} \\
\hline & Absolute uncertainty, ${ }^{\circ} \mathrm{C}$ & $18-38$ & \multicolumn{2}{|c|}{$24-42$} & \multicolumn{2}{|c|}{$31-50$} \\
\hline \multirow{5}{*}{$\begin{array}{c}5 \\
\text { (smallest } \\
\text { control gas } \\
\text { gap) }\end{array}$} & Dominant parameter & Control gas gap & \multicolumn{2}{|c|}{ Fuel heat rate } & \multicolumn{2}{|c|}{ Fuel heat rate } \\
\hline & Dominant sensitivity & $0.32-0.39$ & \multicolumn{2}{|c|}{$0.42-0.60$} & \multicolumn{2}{|c|}{$0.44-0.56$} \\
\hline & Dominant weighted variance, $\%$ & $6.0-9.2$ & \multicolumn{2}{|c|}{$4.6-8.7$} & \multicolumn{2}{|c|}{$4.7-8.1$} \\
\hline & Relative uncertainty, $\%$ & $3.3-4.5$ & \multicolumn{2}{|c|}{$3.0-4.2$} & \multicolumn{2}{|c|}{$3.3-4.2$} \\
\hline & Absolute uncertainty, ${ }^{\circ} \mathrm{C}$ & $30-46$ & \multicolumn{2}{|c|}{$32-52$} & \multicolumn{2}{|c|}{$38-60$} \\
\hline \multirow{5}{*}{$\begin{array}{c}3 \\
\text { (largest } \\
\text { control gas } \\
\text { gap) }\end{array}$} & Dominant parameter & Fuel heat rate & \multicolumn{2}{|c|}{ Fuel heat rate } & \multicolumn{2}{|c|}{ Fuel heat rate } \\
\hline & Dominant sensitivity & $0.30-0.50$ & \multicolumn{2}{|c|}{$0.34-0.55$} & \multicolumn{2}{|c|}{$0.34-0.55$} \\
\hline & Dominant weighted variance, $\%$ & $2.0-6.3$ & \multicolumn{2}{|c|}{$3.0-8.0$} & 3.0 & 8.0 \\
\hline & Relative uncertainty, $\%$ & $2.1-3.4$ & 2.2 & & 2.7 & 3.7 \\
\hline & Absolute uncertainty, ${ }^{\circ} \mathrm{C}$ & $17-33$ & & & & \\
\hline 2 & Dominant parameter & Fuel heat rate & Fuel 1 & at rate & Fuel 1 & at rate \\
\hline (hottest & Dominant sensitivity & $0.38-0.52$ & 0.42 & 0.60 & 0.43 & 0.57 \\
\hline & Dominant weighted variance, $\%$ & $3.7-7.0$ & 4.6 & & 4.7 & 8.2 \\
\hline & Relative uncertainty, $\%$ & $2.3-3.6$ & 2.6 & & 3.0 & 3.9 \\
\hline & Absolute uncertainty, ${ }^{\circ} \mathrm{C}$ & $26-45$ & & & & \\
\hline Capsule & Time-avera & temperature at $\mathrm{t}$ & a or & $\mathrm{R}-2$ & & \\
\hline & & & $\%$ & ${ }^{\circ} \mathrm{C}$ & $\%$ & ${ }^{\circ} \mathrm{C}$ \\
\hline 6 & Overall uncertainty & - & 2.9 & 32 & 3.4 & 40 \\
\hline 5 & Overall uncertainty & - & 3.5 & 39 & 3.7 & 45 \\
\hline 3 & Overall uncertainty & - & 2.7 & 28 & 3.1 & 34 \\
\hline 2 & Overall uncertainty & - & 3.0 & 37 & 3.3 & 45 \\
\hline
\end{tabular}


The daily overall uncertainties in terms of standard deviation for TC temperature, instantaneous and time-average VA and peak fuel temperatures in Capsule 5, as a function of EFPD, are presented in Figure S-6, Figure S-7, Figure S-8 and Figure S-9 show Capsule 5 daily calculated temperatures together with associated error bars of one standard deviation for all temperatures of interest. These plots are similar for other capsules. Result highlights are:

- In general, the overall temperature uncertainties are higher around the middle of irradiation due to higher sensitivities of fuel heat rate (dominant factor) and fuel and graphite thermal conductivities, which are associated with the peak in fuel heat rate. This observation is more profound for temperatures in capsules with larger gas gap distance.

- The drops in temperature uncertainty around 300 EFPD (at the end of ATR Cycle 150B) and 350 EFPD (during ATR Cycle 151A) occurred when the experiment was cooled with pure helium, resulting in low neon fraction uncertainty. The drop in temperature uncertainty around 450 EFPD (PALM Cycle 153B) occurred when the experiment was moved to the peripheral I-24 location and was cooled with pure helium, resulting in low neon fraction uncertainty and low heat rate sensitivity.

- For temperatures at TCs, the overall uncertainty ranged from $2.1 \%$ to $4.5 \%$. The highest relative uncertainty at the end of AGR-2 is $4.5 \%\left(\sim 46^{\circ} \mathrm{C}\right)$ for the peripheral TC in Capsule 5. This high TC uncertainty is caused mainly by the high uncertainty of the small control gas gap distance. The adequacy of basing the uncertainty analysis on model input parameters is confirmed because the overall model uncertainty of predicted TC temperatures is consistent with TC residual variation that ranged from $19^{\circ} \mathrm{C}$ to $30^{\circ} \mathrm{C}$ for one TC in each capsule.

- For instantaneous fuel temperatures, the VA temperature uncertainties are generally lower than peak temperature uncertainties: the relative uncertainty ranged from $2.2 \%$ to $4.2 \%$ for VA temperatures (up to $\sim 52^{\circ} \mathrm{C}$ ), and from $2.7 \%$ to $4.2 \%$ for peak temperatures (up to $\sim 60^{\circ} \mathrm{C}$ ). The fuel temperature uncertainty reaches its highest value during the middle of irradiation when the peak fuel heat rate leads to a high sensitivity coefficient, causing large variations in the calculated fuel temperatures. At the same time, the increase in neon fraction uncertainty due to gas line cross-talk failure during ATR Cycle $150 \mathrm{~B}$ leads to a $5^{\circ} \mathrm{C}$ increase in instantaneous fuel temperature uncertainty.

- For time-average fuel temperatures, Capsule 5 has the highest overall uncertainty because of high control gap uncertainty. The uncertainty reaches $3.5 \%\left(\sim 39^{\circ} \mathrm{C}\right)$ for VA fuel temperature and reaches $3.7 \%\left(\sim 45^{\circ} \mathrm{C}\right)$ for peak fuel temperature near the end of irradiation. Notably, the increase in neon fraction uncertainty during ATR Cycle 150B leads to an increase in instantaneous fuel temperature uncertainty, but has very little impact on the time-average fuel temperature at the end of irradiation.

- The overall uncertainties of the AGR-2 calculated temperatures are comparable to those seen in AGR-1. For instantaneous fuel temperatures, the relative input uncertainty in AGR-1 ranged from $3 \%$ to $4 \%$ for VA temperatures and ranged from 3\% to 5\% for peak temperatures (up to $\sim 65^{\circ} \mathrm{C}$ ). However, the $10 \%$ bias in the AGR-1 Capsule 6 fuel heat rate increased the overall uncertainty in that capsule to $5.8 \%$. For time-average fuel temperatures at the end of irradiation, the overall uncertainty in AGR-1 was 5.0\% for Capsule 6. 


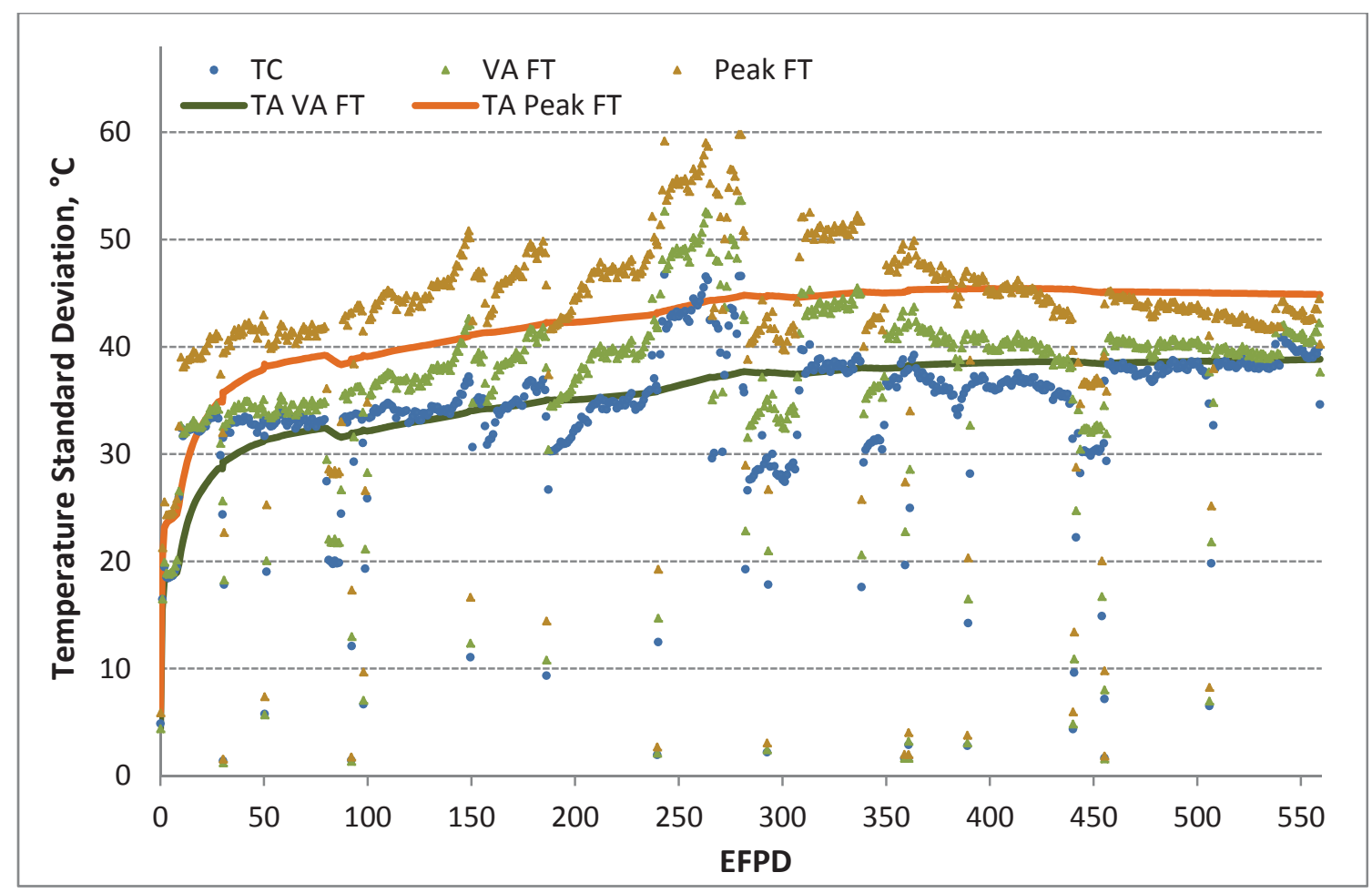

Figure S-6. Daily temperature variances due to input uncertainties in Capsule 5.

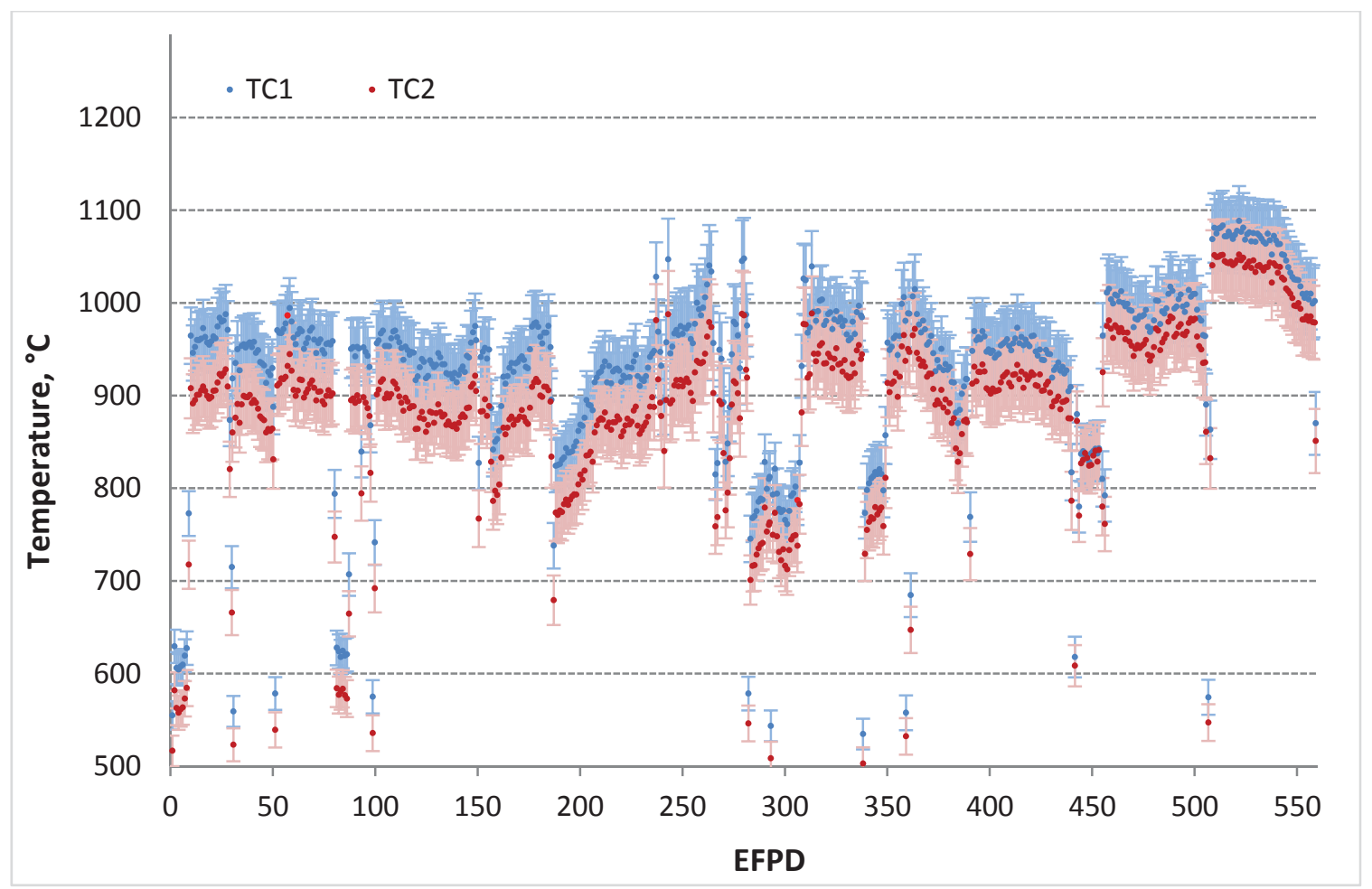

Figure S-7. Instantaneous thermocouple temperatures and uncertainty bars in Capsule 5. 


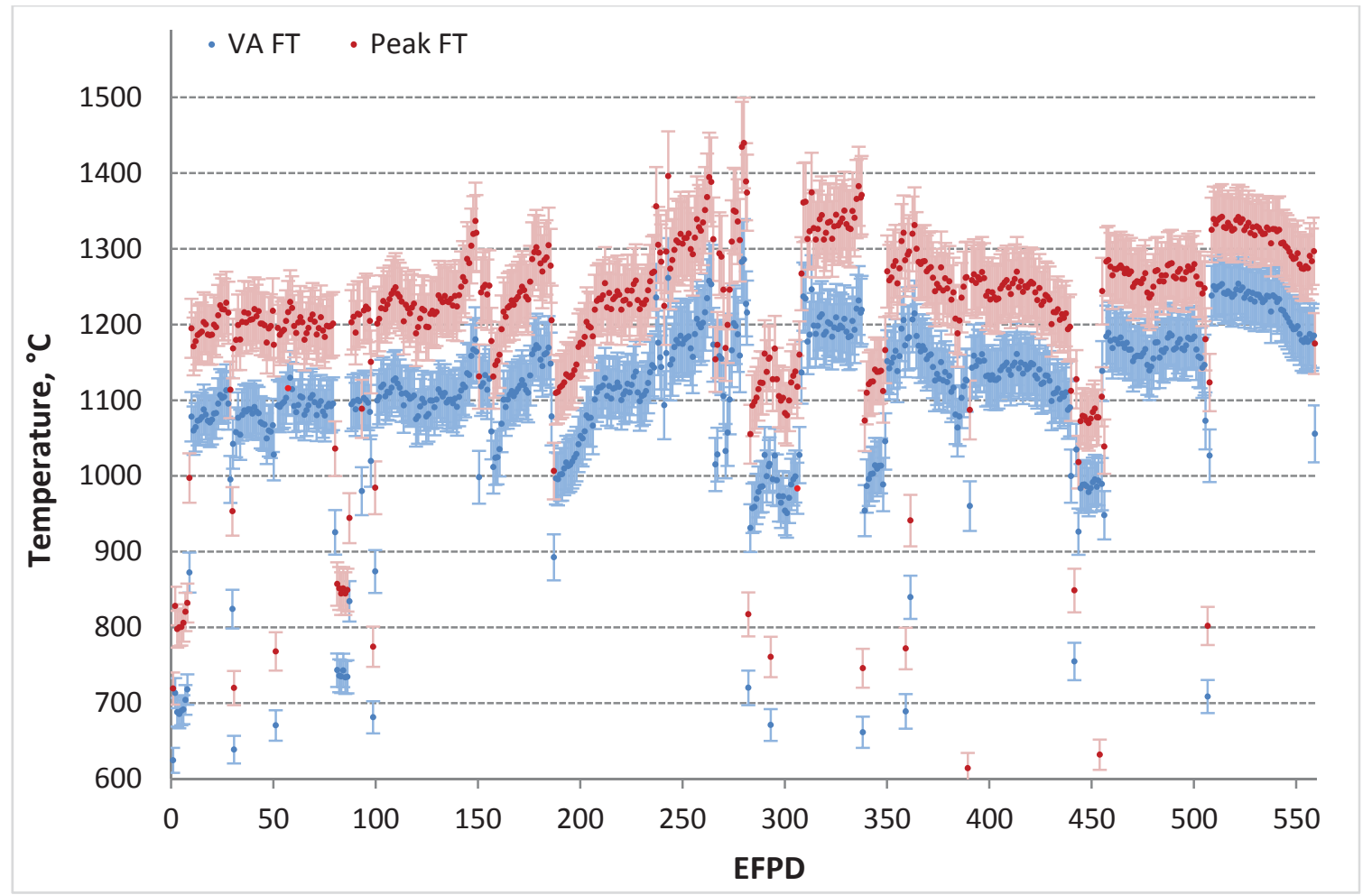

Figure S-8. Instantaneous volume average and peak fuel temperatures and uncertainty bars in Capsule 5.

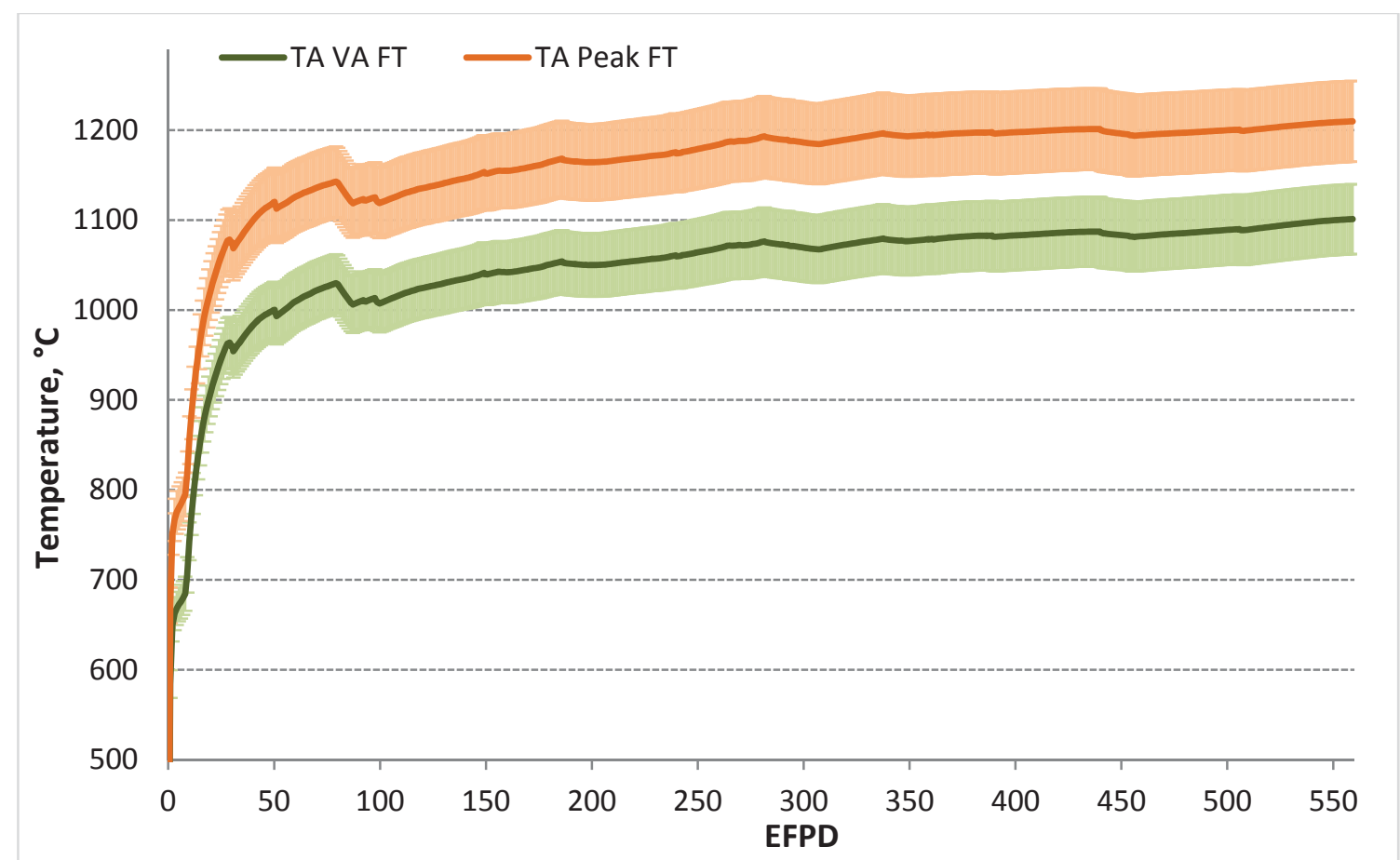

Figure S-9. Time-average, volume average, and peak fuel temperature uncertainties in Capsule 5. 


\section{S-7. Report Structure}

The report is organized into four sections and a conclusion:

- Section 1 introduces the AGR Fuel Development and Qualification Program and summarizes AGR-2 measured data, test configuration and test procedure, and thermal simulation.

- Section 2 describes the estimation of uncertainties and sensitivities for the thermal model input parameters. This includes parameter uncertainties based on expert judgment, sensitivity analysis of input parameters, and the estimation of correlation coefficients for pairs of input parameters.

- Section 3 describes the propagation of uncertainties and sensitivities for estimation of the overall uncertainty for the daily VA and peak fuel temperatures, daily average temperatures at TC locations, and time-average VA and time-average peak fuel temperatures.

- Section 4 presents the results of the uncertainty analysis for each of the six AGR-2 capsules and discusses the parameters driving the uncertainty.

- The conclusion summarizes important findings of the uncertainty quantification and identifies possible areas of improvement. 


\section{CONTENTS}

ABSTRACT vii

SUMMARY ix

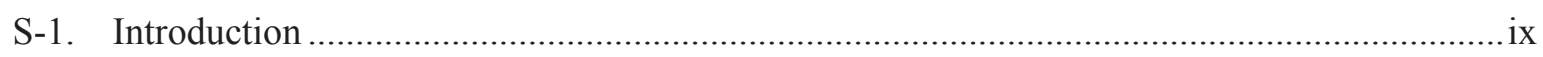

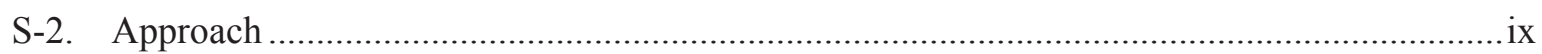

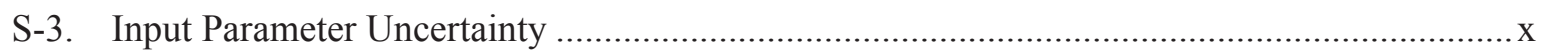

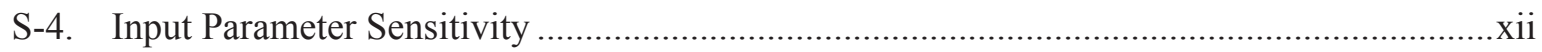

S-5. Combining Parameter Sensitivity and Uncertainty .........................................................iii

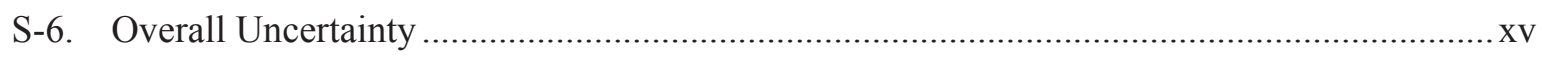

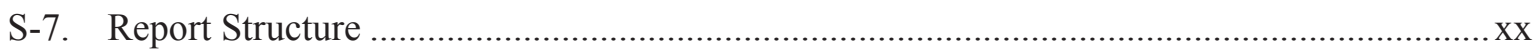

ACRONYMS

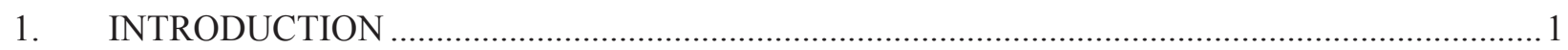

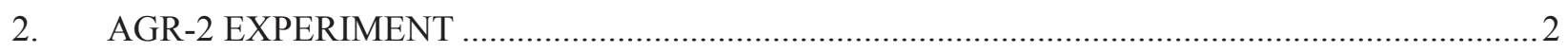

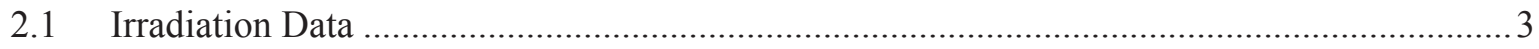

2.2 Thermal Model for AGR-2 Capsules ..................................................................... 4

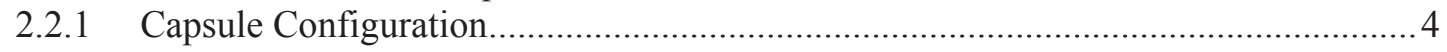

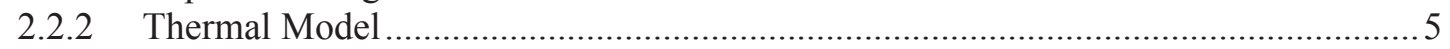

3. UNCERTAINTY QUANTIFICATION OF MODEL PREDICTED TEMPERATURE .................. 9

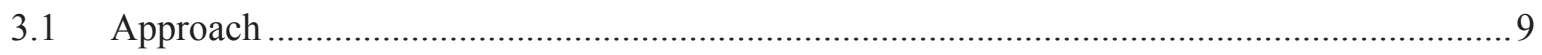

3.1.1 Overall Uncertainty of AGR-2 Predicted Temperatures ........................................... 9

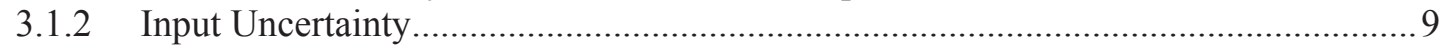

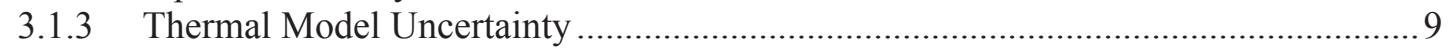

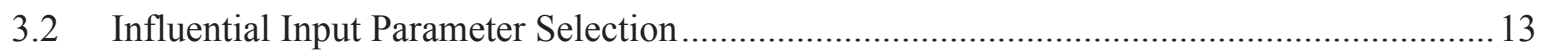

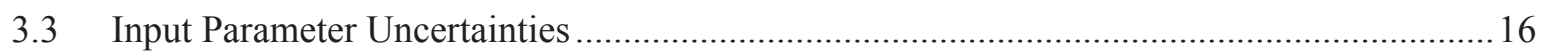

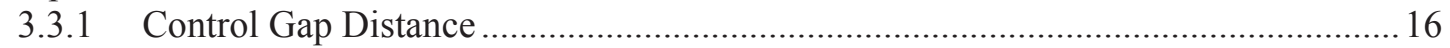

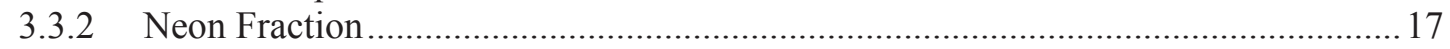

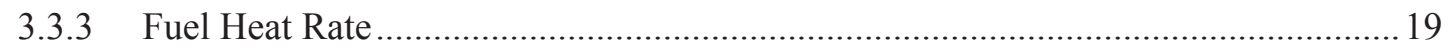

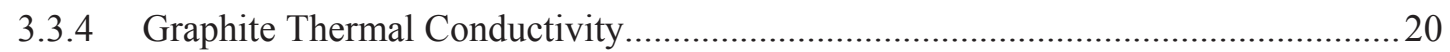

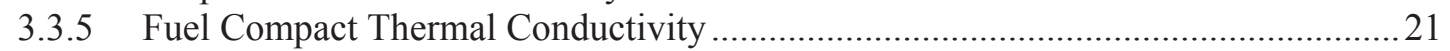

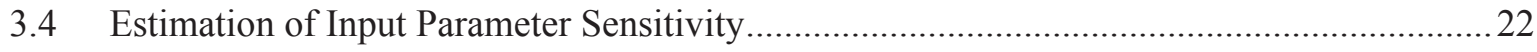

3.4.1 Statistical Experimental Design for Sensitivity Analysis..........................................22

3.4.2 Thermal Model Parameter Sensitivity Analysis.......................................................24

3.4.3 Interpolation of Sensitivity Coefficients for the Entire AGR-2 Irradiation...............29

3.5 Correlation Coefficients of Thermal Model Input Parameters ............................................. 32

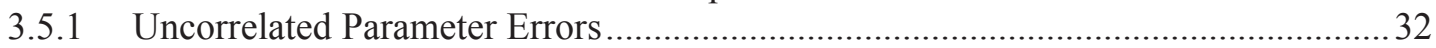

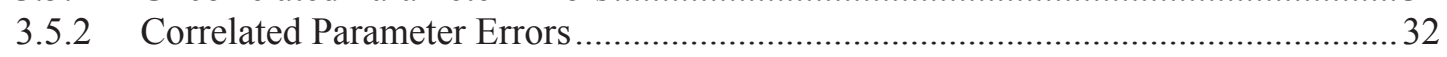

4. PROPAGATION OF PARAMETER UNCERTAINTIES AND SENSITIVITIES .......................36

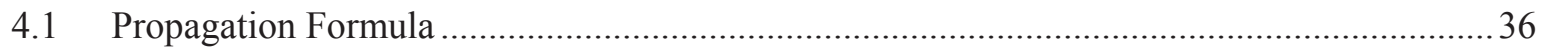

4.2 Uncertainty Quantification for Daily Average Temperatures ........................................... 36

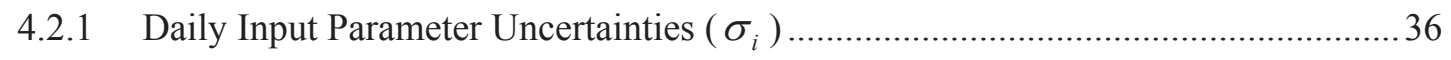




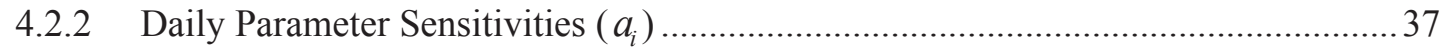

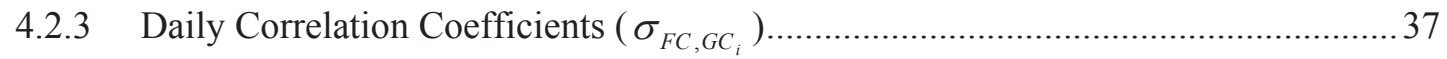

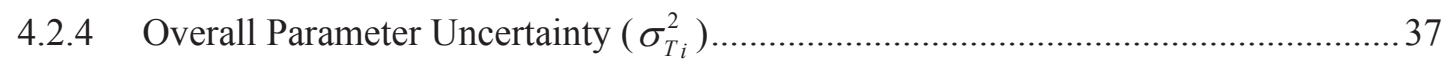

4.3 Uncertainty Quantification for Time Average (TA) Temperatures..................................... 37

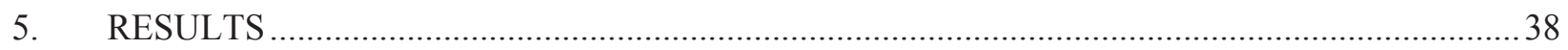

5.1 Temperature Uncertainty Results for Capsule 6 ............................................................. 38

5.2 Temperature Uncertainty Results for Capsule 5 ............................................................. 44

5.3 Temperature Uncertainty Results for Capsule 3 ................................................................50

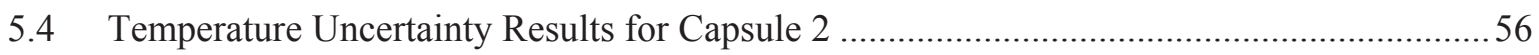

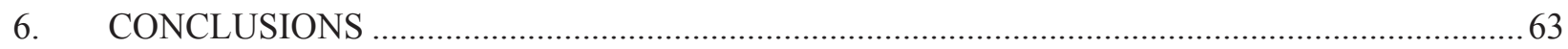

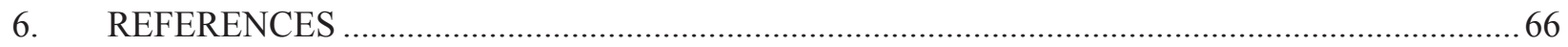

\section{FIGURES}

Figure S-1. Physical sketch of the axial cut of an AGR-2 Capsules. ..................................................

Figure S-2. Capsule 5 input parameter uncertainties in terms of relative standard deviation.................xi

Figure S-3. Parameter sensitivity coefficients for fuel and thermocouple temperatures in

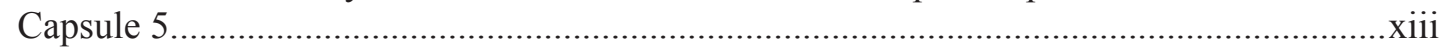

Figure S-4. Capsule 5 daily weighted temperature variances due to parameter uncertainties. .............xiv

Figure S-5. Capsule 2 daily weighted temperature variances due to parameter uncertainties............... XV

Figure S-6. Daily temperature variances due to input uncertainties in Capsule 5.............................xviii

Figure S-7. Instantaneous thermocouple temperatures and uncertainty bars in Capsule 5................xviii

Figure S-8. Instantaneous volume average and peak fuel temperatures and uncertainty bars in

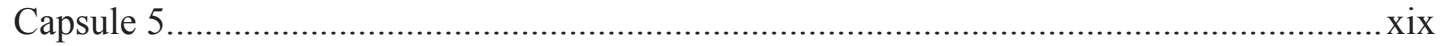

Figure S-9. Time-average, volume average, and peak fuel temperature uncertainties in Capsule 5. xix

Figure 1. AGR-2 location in the ATR core cross section. 2

Figure 2. Three-dimensional view of a single AGR-2 capsule and axial cross-section view of the six capsules in an AGR-2 experiment. 3

Figure 3. Graphical summary of Capsule 5 data during AGR-2 test. .................................................. 4

Figure 4. Schematic of a radial cut of an AGR-2 capsule .................................................................. 5

Figure 5. Physical sketch of the axial cut of an AGR-2 capsules........................................................ 6

Figure 6. Temperature distribution in cutaway view of three fuel stacks. .......................................... 7

Figure 7. Calculated temperature history of daily minimum, maximum, and volume average for AGR-2 Capsules 6, 5, 3, and 2...... 8 
Figure 8. The thermocouple residuals of the original (run 1) and updated (run 2) thermal models for AGR-1 Capsules 4 and 6.

Figure 9. Difference between measured and predicted thermocouple temperatures for AGR-2 U.S. capsules

Figure 10. Histograms of temperature residuals of peripheral TCs of four U.S. capsules.................... 13

Figure 11. Parameter sensitivities for Capsule 5 peak and VA fuel temperatures............................... 14

Figure 12. Parameter sensitivities for Capsule 5 calculated TC temperature....................................... 15

Figure 13. The initial and end gap distance with an error bar of 1 mil for four U.S. capsules. ............. 17

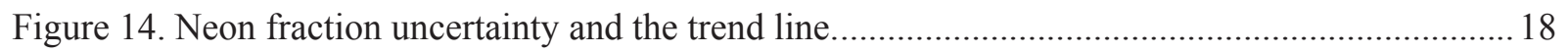

Figure 15. Capsule neon fractions after 150A PALM cycle. The neon fractions for all capsules were set to the same value beginning January 17, 2012 (ATR Cycle 151A). ....................... 19

Figure 16. Capsule average power density versus irradiation time in EFPD......................................20

Figure 17. Ratio of irradiated over unirradiated graphite thermal conductivity $\left(\mathrm{k}_{\mathrm{irr}} / \mathrm{k}_{\mathrm{o}}\right)$ varying with temperature and dpa.

Figure 18. UCO compact thermal conductivity varying with fluence and temperature (ECAR2476). .22

Figure 19. Actual by predicted plot for VA FT for Capsule 4.

Figure 20. Parameter estimates (Equation (10)) sorted from the largest to the smallest for VA fuel temperature of Scenario 1.

Figure 21. Leverage plots for five main effects and the square term of Ne fraction for VA FT (parameters are relative ratios to their nominal values).

Figure 22. Prediction profiles of functions for VA fuel, peak fuel, and TC temperatures....................28

Figure 23. Sensitivity coefficients for fuel and TC temperatures as function of EFPD......................22

Figure 24. Heat rate sensitivity for thermocouples and fuel temperatures as function of heat rate.

Figure 25. Neon fraction sensitivity for thermocouples and fuel temperatures as function of neon fraction.

Figure 26. Gas gap sensitivity for thermocouples and fuel temperatures as function of gap distance.

Figure 27. Graphite conductivity sensitivity for thermocouples and fuel temperatures as function of fluence.

Figure 28. Fuel conductivity sensitivity for thermocouples and fuel temperatures as function of fluence.

Figure 29. Scatter plot matrix shows the correlation between fuel and graphite thermal conductivities at one fuel temperature and two $d p a$ levels (0.5 and 2.0).

Figure 30. Correlation coefficients between graphite and fuel thermal conductivities varying with fast neutron fluence. .35

Figure 31. Daily input relative standard deviations for Capsule 6. .39

Figure 32. Daily parameter sensitivities for temperatures in Capsule 6. 40

Figure 33. Daily temperature variances due to input uncertainties in Capsule 6. 
Figure 34. Capsule 6 temperature uncertainties in terms of relative standard deviations.

Figure 35. Daily standard deviations of predicted thermocouple and fuel temperatures in Capsule 6. 42

Figure 36. Model temperature and standard deviation of thermocouples in Capsule 6.

Figure 37. Model temperature and standard deviation of daily averaged fuel temperatures in Capsule 6.

Figure 38. Model temperature and standard deviation of time-average fuel temperatures in Capsule 6. .44

Figure 39. Daily input relative standard deviations for Capsule 5. 45

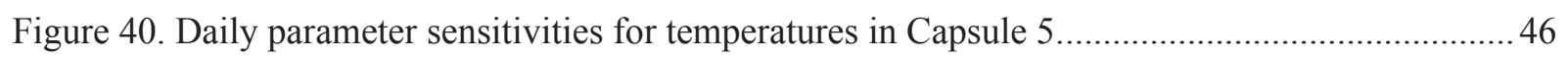

Figure 41. Daily temperature variances due to input uncertainties in Capsule 5 ................................47

Figure 42. Capsule 5 temperature uncertainties in terms of relative standard deviations.....................48

Figure 43. Daily standard deviations of predicted thermocouple and fuel temperatures in Capsule 5 .48

Figure 44. Model temperature and standard deviation of thermocouples in Capsule 5.

Figure 45. Model temperature and standard deviation of daily averaged fuel temperatures in Capsule 5

Figure 46. Model temperature and standard deviation of time-average fuel temperatures in Capsule 5 50

Figure 47. Daily input relative standard deviations for Capsule 3...... .51

Figure 48. Daily parameter sensitivities for temperatures in Capsule 3 ............................................52

Figure 49. Daily temperature variances due to input uncertainties in Capsule 3................................53

Figure 50. Capsule 3 temperature uncertainties in terms of relative standard deviations.....................54

Figure 51. Daily standard deviations of predicted thermocouple and fuel temperatures in Capsule 3 .54

Figure 52. Model temperature and standard deviation of thermocouples in Capsule 3. .55

Figure 53. Model temperature and standard deviation of daily averaged fuel temperatures in Capsule 3

Figure 54. Model temperature and standard deviation of time-average fuel temperatures in Capsule 3 56

Figure 55. Daily input relative standard deviations for Capsule 2 ...............................................57

Figure 56. Daily parameter sensitivities for temperatures in Capsule 2 .............................................58

Figure 57. Daily temperature variances due to input uncertainties in Capsule 2.................................59

Figure 58. Capsule 2 temperature uncertainties in terms of relative standard deviations.....................60

Figure 59. Daily standard deviations of predicted TC and fuel temperatures in Capsule 2.................6 60

Figure 60. Model temperature and standard deviation of TCs in Capsule 2 ...................................... 61

Figure 61. Model temperature and standard deviation of daily averaged fuel temperatures in Capsule 2. 
Figure 62. Model temperature and standard deviation of time-average fuel temperatures in Capsule 2 .

\section{TABLES}

Table S-1. Uncertainties of the most significant parameters of the AGR-2 thermal model....................xi

Table S-2. Temperature uncertainty results for U.S. AGR-2 capsules. ............................................. xvi

Table 1. Uncertainties of the most significant parameters of the AGR-2 thermal model..................... 15

Table 2. Control gap distance variation and uncertainty for AGR-2 capsules.................................... 17

Table 3. Experimental design matrix for AGR-2 thermal model sensitivity analysis...........................23

Table 4. AGR-2 Capsule 5 thermal conditions for selected scenarios..................................................24

Table 5. Parameter estimates (sensitivity) for VA FT, peak FT and TC1 temperatures.......................26

Table 6. Sensitivity coefficients of fuel heat rate for thermocouples, volume average fuel, and

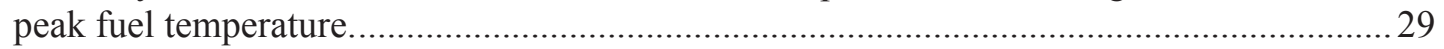

Table 7. Correlation coefficients between graphite and fuel thermal conductivities........................... 35

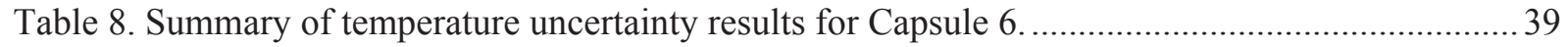

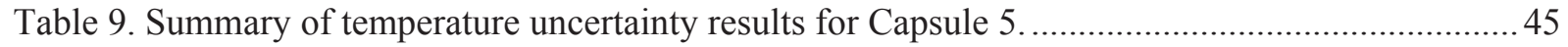

Table 10. Summary of temperature uncertainty results for Capsule 3 ...............................................51

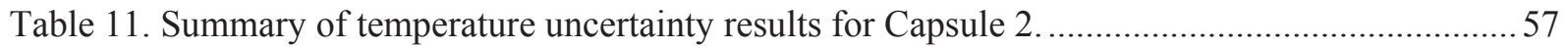


xxvi 


\section{ACRONYMS}

$\begin{array}{ll}\text { AGR } & \text { Advanced Gas Reactor } \\ \text { ART } & \text { Advanced Reactor Technology } \\ \text { ATR } & \text { Advanced Test Reactor } \\ \text { dpa } & \text { displacements per atom } \\ \text { EFPD } & \text { effective full-power day } \\ \text { FC } & \text { fuel compact } \\ \text { FT } & \text { fuel temperature } \\ \text { GC } & \text { graphite conductivity } \\ \text { GG } & \text { gas gap } \\ \text { HR } & \text { heat rate } \\ \text { INL } & \text { Idaho National Laboratory } \\ \text { NDMAS } & \text { Nuclear Data Management and Analysis System } \\ \text { PIE } & \text { post-irradiation examination } \\ \text { R\&D } & \text { research and development } \\ \text { TA } & \text { time average } \\ \text { TC } & \text { thermocouple } \\ \text { TRISO } & \text { tristructural-isotropic } \\ \text { VA } & \text { volume average }\end{array}$


xxviii 


\section{Uncertainty Quantification of Calculated Temperatures for the U.S. Capsules in the AGR-2 Experiment}

\section{INTRODUCTION}

A series of Advanced Gas Reactor (AGR) irradiation experiments are being conducted within the Advanced Reactor Technology (ART) Fuel Development and Qualification Program. The main objectives of the fuel experimental campaign are to provide the necessary data on fuel performance to support fuel process development, qualify a fuel design and fabrication process for normal operation and accident conditions, and support development and validation of fuel performance and fission product transport models and codes (PLN-3636, "Technical Program Plan for the Next Generation Nuclear Plant/Advanced Gas Reactor Fuel Development and Qualification Program"). The AGR-2 test was inserted in the B-12 position in the Advanced Test Reactor (ATR) core at Idaho National Laboratory (INL) in June 2010 and successfully completed irradiation in October 2013, resulting in irradiation of the tristructural-isotropic (TRISO) fuel for 559.2 effective full-power days (EFPDs) during approximately 3.3 calendar years. The AGR-2 data, including the irradiation data and calculated results, were qualified and stored in the Nuclear Data Management and Analysis System (NDMAS) (Pham and Einerson 2014).

To support the U.S. TRISO fuel performance assessment and to provide data for validation of fuel performance and fission product transport models and codes, the daily as-run thermal analysis has been performed separately on each of four AGR-2 U.S. capsules for the entire irradiation as discussed in (ECAR-2476, "AGR-2 Daily As-Run Thermal Analyses"). The ABAQUS code's finite element-based thermal model predicts the daily average volume-average (VA) fuel temperature (FT) and peak FT in each capsule. This thermal model involves complex physical mechanisms (e.g., graphite holder and fuel compact shrinkage) and properties (e.g., conductivity and density). Therefore, the thermal model predictions are affected by uncertainty in input parameters and by incomplete knowledge of the underlying physics leading to modeling assumptions. Therefore, alongside with the deterministic predictions from a set of input thermal conditions, information about prediction uncertainty is instrumental for the ART program decision-making. Well defined and reduced uncertainty in model predictions helps increase the quality of and confidence in the AGR technical findings (Pham et al. 2013, 20134).

The JMOCUP simulation codes were created to perform depletion calculations for the AGR-2 experiment (ECAR-2066, "JMOCUP As-Run Daily Depletion Calculation for the AGR-2 Experiment in ATR B-12 Position"). This depletion analysis provides fast fluence and fission heat rate data for all components (fuel compacts, graphite holders, stainless-steel retainer, etc.) used as inputs for the thermal analysis codes (ECAR-2476). The graphite temperature from thermocouples (TCs) in the AGR capsules was used to calibrate these thermal analysis codes. However, given a high rate of TC failure under the harsh irradiation and thermal conditions in the AGR capsules, the thermal analysis results are very useful in aiding TC data qualification, increasing the confidence in delineating failures of the measuring instruments (TCs) from physical mechanisms that may have shifted the system thermal response (Pham and Einerson 2011). Thus, the combined use of test data and simulation results requires a systematic approach to uncertainty quantification of both experimental measurements and model predictions.

This report focuses on the uncertainty quantification of TC and FTs predicted by the ABAQUS-based thermal models for four U.S. capsules in AGR-2 experiment. Because of Cooperative Research and Development Agreement restrictions, discussion about data from Capsule 1 (the South African capsule) and Capsule 4 (the French capsule) is not presented here. To quantify the uncertainty of AGR calculated temperatures, ABAQUS thermal model input parameters of potential importance are identified. Identification has two parts: (1) using expert judgment, determine parameters with the largest uncertainties and estimate these uncertainties, and (2) using sensitivity analysis, determine parameters that 
the modeling is most sensitive to. A set of parameters is selected for predicted temperature uncertainty quantification, including those with high sensitivity and those with large uncertainty. The parameter uncertainties and sensitivity coefficients are combined and propagated to quantify the overall uncertainty of temperature outputs.

\section{AGR-2 EXPERIMENT}

The primary objectives of the AGR-2 experiment are defined in PLN-3636 and a detailed description of the experiment is provided in PLN-3798, "AGR-2 Irradiation Experiment Test Plan." The AGR-2 irradiation demonstrates performance of U.S. TRISO fuel particles containing UCO (uranium oxycarbide) and $\mathrm{UO}_{2}$ (uranium dioxide) fuel produced in a large (i.e., 15.24-cm) coater. The AGR-2 test train was inserted into the large B-12 location of the ATR core (Figure 1). The AGR-2 test train is comprised of six individual capsules, approximately $3.49 \mathrm{~cm}$ diameter and $15.24 \mathrm{~cm}$ long (see the left figure of Figure 2), stacked on top of each other. The capsules are numbered consecutively from the bottom (Capsule 1) to the top (Capsule 6) as shown on the right of Figure 2. Each capsule consists of fuel compacts placed in a graphite cylinder shrouded by a hafnium and stainless steel layer and capsule shell. A leadout tube holds the experiment in position and contains and protects the gas lines and TC wiring extending from the test train to the reactor penetration. Each AGR-2 capsule contains only one type of TRISO-coated fuel particles. U.S. UCO fuel particles are in Capsules 2, 5, and 6; U.S. $\mathrm{UO}_{2}$ fuel particles are in Capsule 3; French $\mathrm{UO}_{2}$ fuel particles are in Capsule 1; and South African $\mathrm{UO}_{2}$ fuel particles are in Capsule 4. The French and South African capsule data are not presented or discussed in this report because of CRADA restrictions.

For test fuel temperature control, the AGR experiments are instrumented with TCs terminating in the graphite sample holder of each capsule. A total of 15 TCs are installed in the AGR-2 experiment; five TCs are located in Capsule 6 and two TCs in each of the remaining capsules. The TCs have an installed accuracy of $\pm 2 \%$ of readings as required by the test specification (SPC-1064). The target quantity (e.g., fuel temperature) is regulated by the helium-neon gas mixture that flows through the control gap between the graphite holder and retainer, and gaps between fuel compacts and the graphite holder holes.

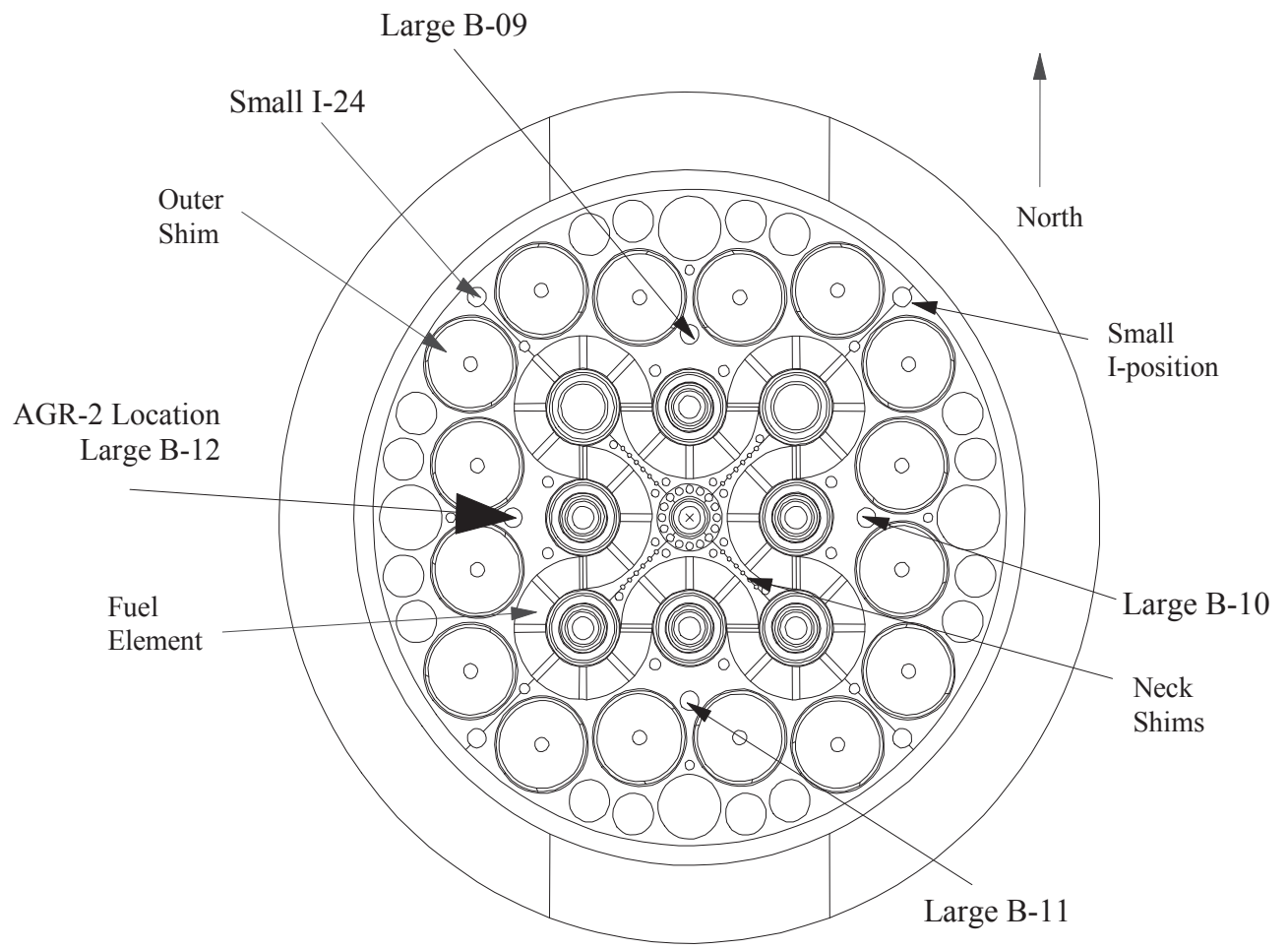

Figure 1. AGR-2 location in the ATR core cross section. 


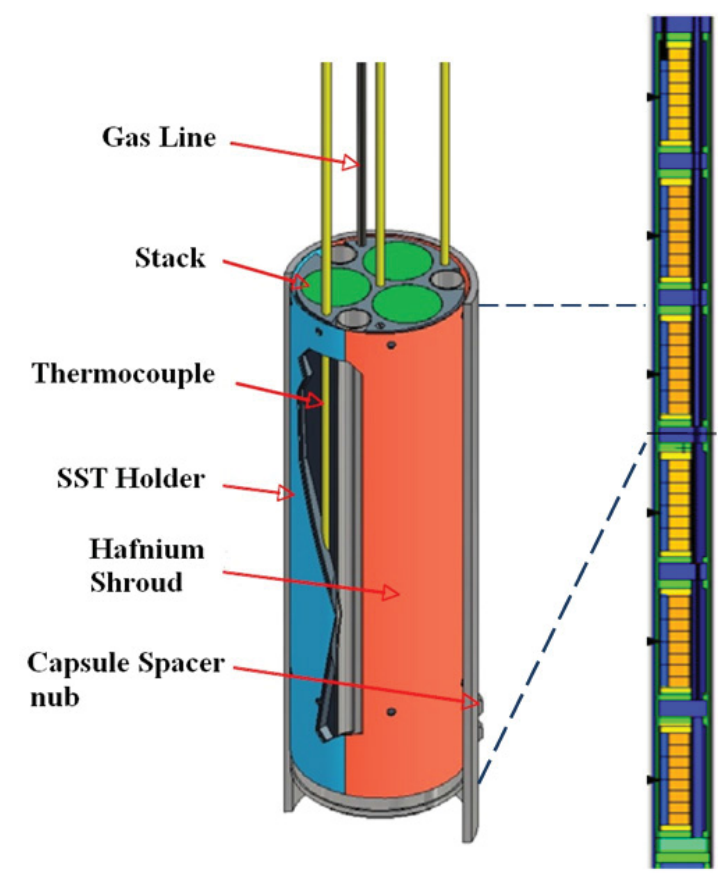

Figure 2. Three-dimensional view of a single AGR-2 capsule and axial cross-section view of the six capsules in an AGR-2 experiment.

\subsection{Irradiation Data}

The AGR-2 irradiation was started in June 2010 (ATR Cycle 147A) and completed in October 2013 (end of ATR Cycle 154B) resulting in 559.2 effective full power days (EFPD). The AGR-2 experimental data, consisting of fabrication data, irradiation data including fission product monitoring, and post-irradiation examination (PIE) data were collected, scrutinized and stored in the NDMAS. The graphical plots in Figure 3 summarize Capsule 5 measured and calculated data, which are related to thermal condition, as a function of AGR-2 irradiation EFPDs. The data plotted here are daily averaged values: TC measurements are in the first panel, calculated TC and fuel compact temperatures are in Panels 2 and 3, and three important inputs to capsule thermal model (i.e., neon fraction of gas mixture, fuel compact heat rate, and fuel compact fast fluence) are in the bottom panels. The neon fraction of gas mixture and fuel compact heat rate directly determines capsule temperature. The fast fluence is included here because of its influence on fuel compact and graphite holder conductivities as well as on the control gas gap distance.

The number of TCs in capsules and the diameter of their wires are limited by space in the test train through-tubes. These TCs were selected based on the greatest survival probability and least amount of drift in accelerated furnace tests; however, TC failures are still expected because the high temperatures and high neutron fluence exposure during extended irradiation far exceed vendor specifications. By the end of Cycle 154A, all TCs in the AGR-2 test train failed due to instrument failures. Capsule 5 lost both TCs by the end of ATR Cycle 149A as shown in the first frame of Figure 3. The accuracy of TC readings could be assessed by comparison with the readings from other TCs in similar positions or with calculated values (Pham, Einerson 2011). The TC data are rigorously analyzed and qualified in NDMAS (Pham, Einerson 2014).

The measurements of the designated control TC support temperature control of the experiment. The target fuel temperature is independently regulated by the helium-neon gas mixture that fills the gap volume for each capsule. TC measurements provide feedback to the automated sweep gas mass flow controller (MFC) system for each capsule, which then adjusts gas blend to maintain the reference 
temperature in response to variation of the fuel heat rate (Panel 4). The TC measurements also are used to calibrate the capsule thermal models, which ultimately determine fuel temperatures.

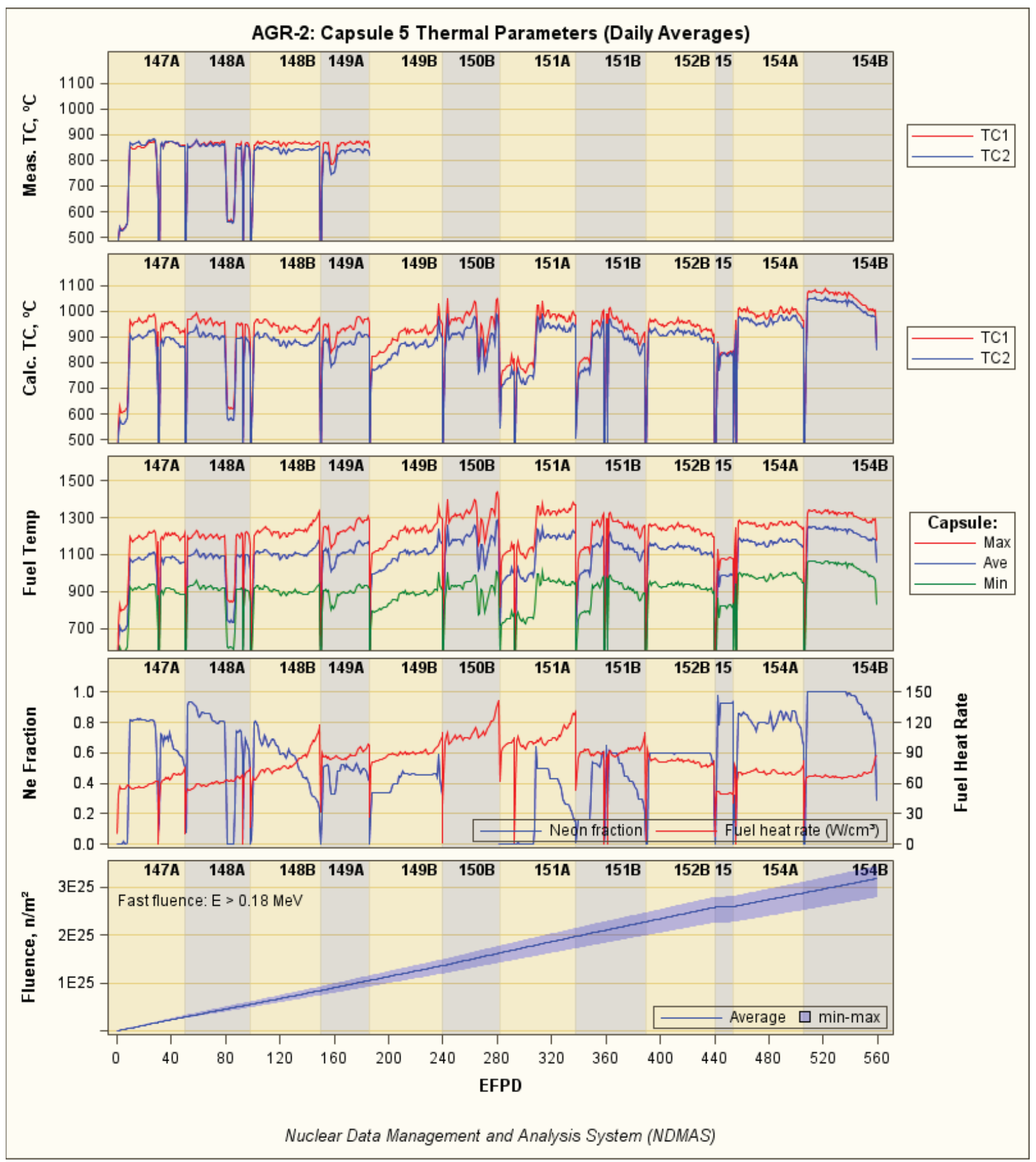

Figure 3. Graphical summary of Capsule 5 data during AGR-2 test.

\subsection{Thermal Model for AGR-2 Capsules}

\subsubsection{Capsule Configuration}

Each AGR-2 capsule contains twelve fuel compacts stacked in three vertical columns of four compacts per stack, which are placed in a graphite cylinder sample holder shrouded by a hafnium and 
stainless steel layer and housed in a capsule body (Figure 4). The TC and gas-line locations are also shown in Figure 4.

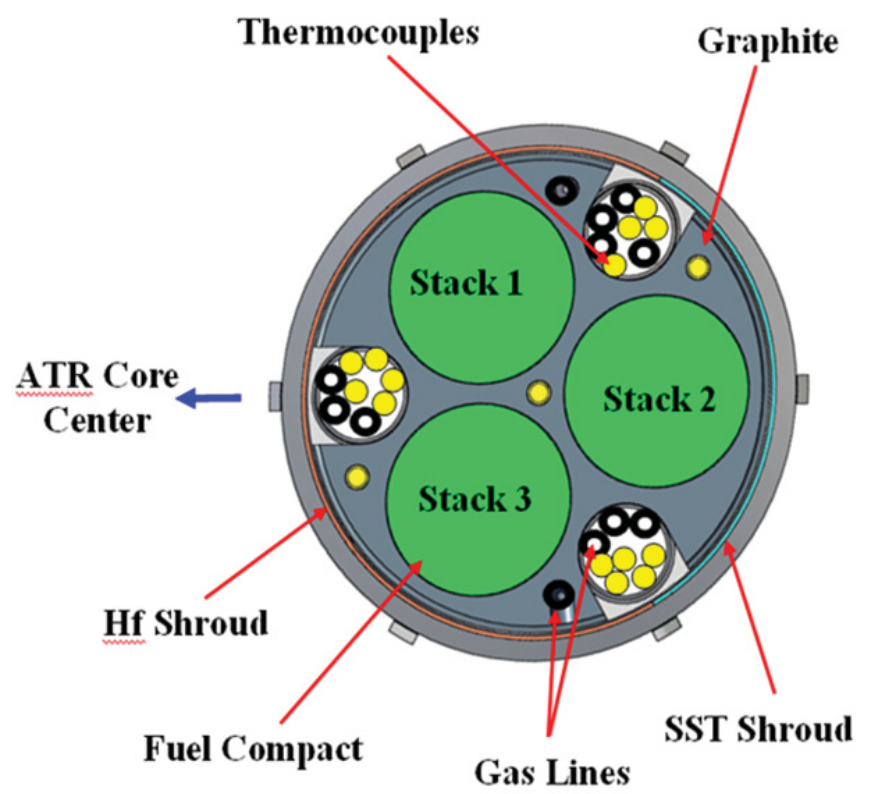

Figure 4. Schematic of a radial cut of an AGR-2 capsule.

\subsubsection{Thermal Model}

Figure 5depicts a physical sketch representing the main parameters of the thermal model for an AGR-2 capsule. The ATR primary cooling water is the ultimate heat sink for each capsule. The fission power predominantly generated in the fuel compact and graphite sample holder is largely conducted out to the ATR primary cooling water through the two gas gaps: one between the fuel stack and the graphite holder hole, and one between the graphite holder and the stainless steel shell (called the control gas gap). The tests are instrumented with TCs embedded in graphite blocks to measure the lower temperature in the graphite. The independently controlled helium-neon gas mixture flows through the gaps to maintain specified TC readings, ensuring that target FTs are within the specification defined by program management (SPC-1064).

ABAQUS-based (Version 6.8-2), three-dimensional finite-element thermal models are created for each capsule of the AGR-2 test to predict daily averages of fuel compact and TC temperatures for the entire irradiation period when the ATR core is at power. The validation of ABAQUS version 6.8-2 was performed and reported in (ECAR-2476). It comprised 10 thermal models validating different aspects of ABAQUS' heat transfer abilities. The maximum difference between ABAQUS calculated values and exact theoretical values is just under $2.0 \%$. The governing equation of steady-state conductive heat transfer is expressed as (ECAR-2476)

$$
\rho c_{p}\left(u_{x} \frac{\partial T}{\partial x}+u_{y} \frac{\partial T}{\partial y}+u_{z} \frac{\partial T}{\partial z}\right)=\frac{\partial}{\partial x}\left(k(T) \frac{\partial T}{\partial x}\right)+\frac{\partial}{\partial y}\left(k(T) \frac{\partial T}{\partial y}\right)+\frac{\partial}{\partial z}\left(k(T) \frac{\partial T}{\partial z}\right)+Q
$$


where $\rho$ is the density; $c_{p}$ is the specific heat; $u_{x}, u_{y}$, and $u_{z}$ are the three-directional velocities; $T$ is the temperature; $x, y$, and $z$ are direction; $k(T)$ is the thermal conductivity varying with temperature and neutron fluence; and $Q$ is the heat source. The heat transfer through the gas gap is by conduction and radiation only; there is no advection, because of the very low flow rate of gas $\left(30 \mathrm{~cm}^{3} / \mathrm{min}\right)$. The governing equation for radiation heat transfer across the control gas gap is

$$
q_{n e t}=\frac{\sigma\left(T_{1}^{4}-T_{2}^{4}\right)}{\frac{\left(1-\varepsilon_{1}\right)}{\varepsilon_{1} A_{1}}+\frac{1}{A_{1} F_{12}}+\frac{\left(1-\varepsilon_{2}\right)}{\varepsilon_{2} A_{2}}}
$$

where $q_{\text {net }}$ is the net heat flux, $\sigma$ is the Stephan Boltzmann constant, $T_{1}$ and $T_{2}$ are the surface temperatures, $\varepsilon_{1}$ and $\varepsilon_{2}$ are the emissivity of Surfaces 1 and 2, $A_{1}$ and $A_{2}$ are the areas of Surfaces 1 and 2 , and $F_{12}$ is the view factor from Surface 1 to 2.

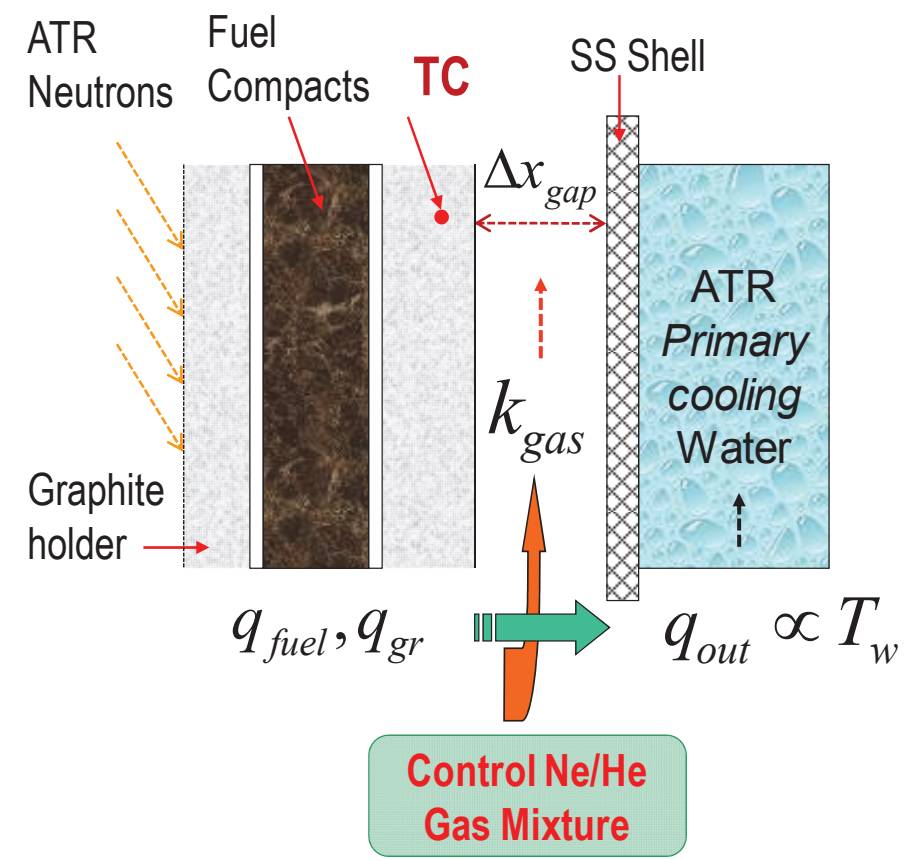

Figure 5. Physical sketch of the axial cut of an AGR-2 capsules.

The main time-series inputs to the model are daily component (namely, fuel compacts and graphite sample holder) heat rates $(Q)$ and neutron fast fluences calculated from the as-run depletion analysis (ECAR-2066) and daily gas compositions of the helium-neon mixture (Ne fraction). The fast neutron fluence is needed for calculation of the components' thermal conductivity and for estimation of the control gas gap distance. The ABAQUS thermal model uses a 350,000 eight-node hexahedral brick finite element mesh to estimate capsule temperature profiles as shown in Figure 6 for three fuel stacks. However, uncertainty remains because of a lack of knowledge about phenomena such as heat- and neutron-induced changes in the capsule control gas gaps. While no direct measurements of FTs are available, the graphite holder temperature from TCs in AGR tests was used to validate computer codes. 
The control gas gaps (i.e., gaps between the graphite holder and stainless steel retainer) and compact-graphite holder gas gaps vary based on fast neutron fluence (Hawkes et al. 2014). The PIE metrology data of AGR-1 capsule components indicated that all fuel compacts shrank unevenly and the graphite sample holders swelled unevenly (because of high boron addition to the graphite) for the four middle Capsules (2-5) with the weight percent (wt\%) boron present in the material of $7 \% \mathrm{~B}_{4} \mathrm{C}$, but shrank for Capsules 1 and 6 (with 5.5\% $\mathrm{B}_{4} \mathrm{C}$ ) (Demkowicz et al. 2011). As a result, the control gap shrank for the four middle capsules and expanded for the top and the bottom capsules; however, the gap between the graphite body and the fuel compact increased, offsetting somewhat the reduction in control gap in calculating FT. Thus, this behavior is taken into account when calculating the AGR-2 temperatures because the control gas gaps and compact-graphite holder gas gaps are assumed to mimic the AGR-1 Capsule 6 gap models because of similar geometry and boron concentration. As a result, the AGR-2 thermal model assumes that the control gap is evenly and linearly increasing for all capsules over the entire AGR-2 irradiation from initial actual hot gap size (subtracting thermal expansion of graphite holder) to the estimated end gap.

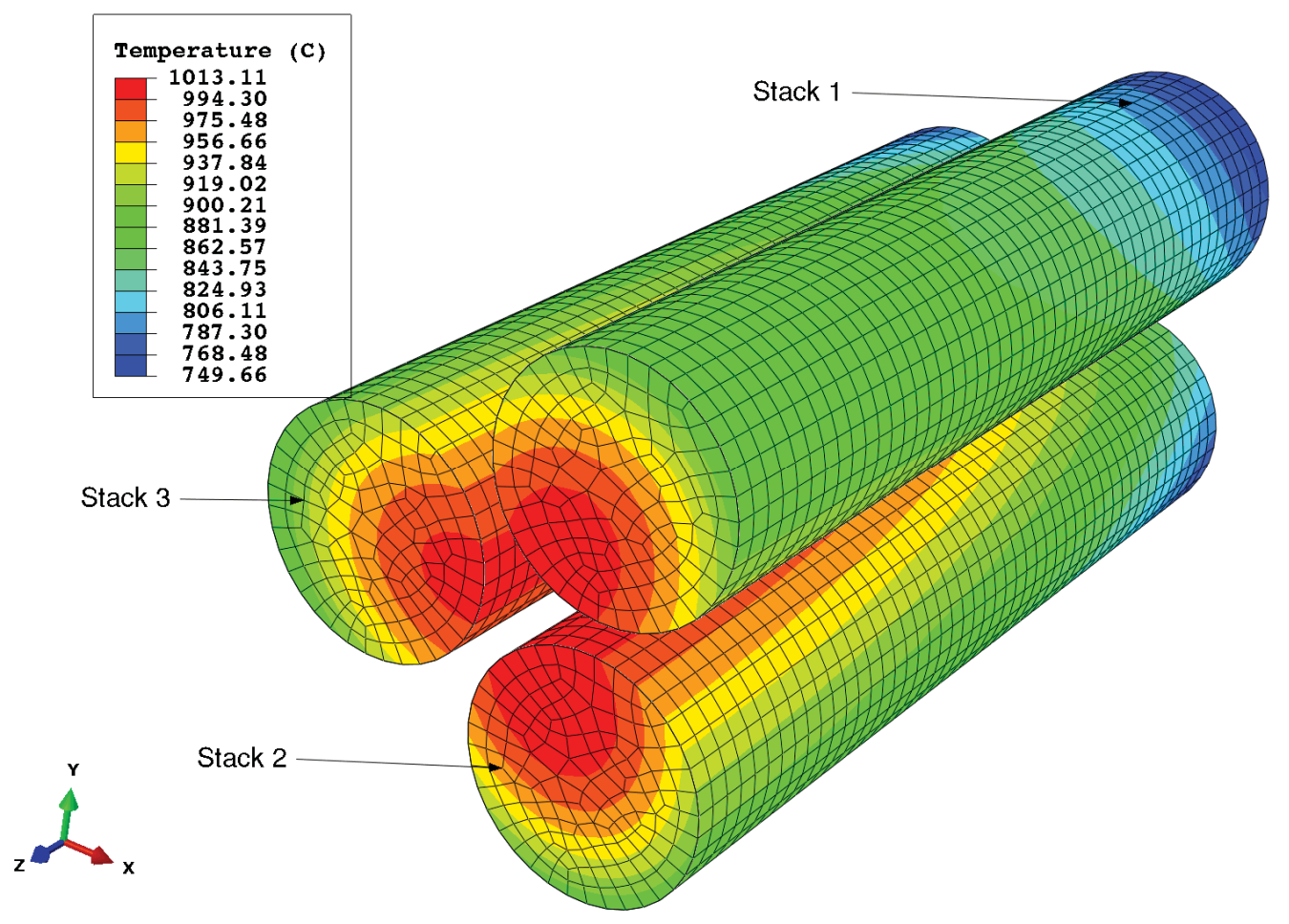

Figure 6. Temperature distribution in cutaway view of three fuel stacks.

Besides the gas gap assumptions, the following factors and model assumptions may also contribute to predicted temperature uncertainties (ECAR-2476):

1. Heat rates from components (excluding fuel compacts divided into two nodes) and fluences are spatially constant and vary only with time for each capsule.

2. Graphite and compact thermal conductivity vary with fluence and temperature, which are taken from legacy experiment correlations and scaled for AGR-2 material density. Graphite conductivity for Capsules 6 and 3 is based on $4.5 \%$ boron, while Capsules 5 and 2 graphite conductivities are based on $5.5 \%$ boron.

3. Gas mixture thermal conductivity is determined by kinetic theory of gases using pure gas properties of helium and neon to determine mixture properties. 
4. Radiation heat transfer occurs from the graphite holder to the stainless-steel retainer, graphite holder to thru-tubes, and thru-tubes to the stainless-steel retainer. An emissivity of 0.4 was assumed for the stainless steel retainer and an emissivity of 1.0 for the graphite and thru tubes. Thru tubes are considered to be covered with graphite dust.

5. There is no axial heat conduction from one capsule to the next.

The example of FT distribution in cutaway view of three fuel stacks presented in Figure 6 is a typical distribution for all capsules and time steps (ECAR-2476). Temperatures range from $750^{\circ} \mathrm{C}$ to a maximum of $1,013^{\circ} \mathrm{C}$. Stacks 1 and 2 have higher temperatures than Stack 3 because they are closer to the core center. Figure 7 shows the calculated daily-average fuel compact maximum, average, and minimum temperature for four U.S. capsules (ECAR-2476).

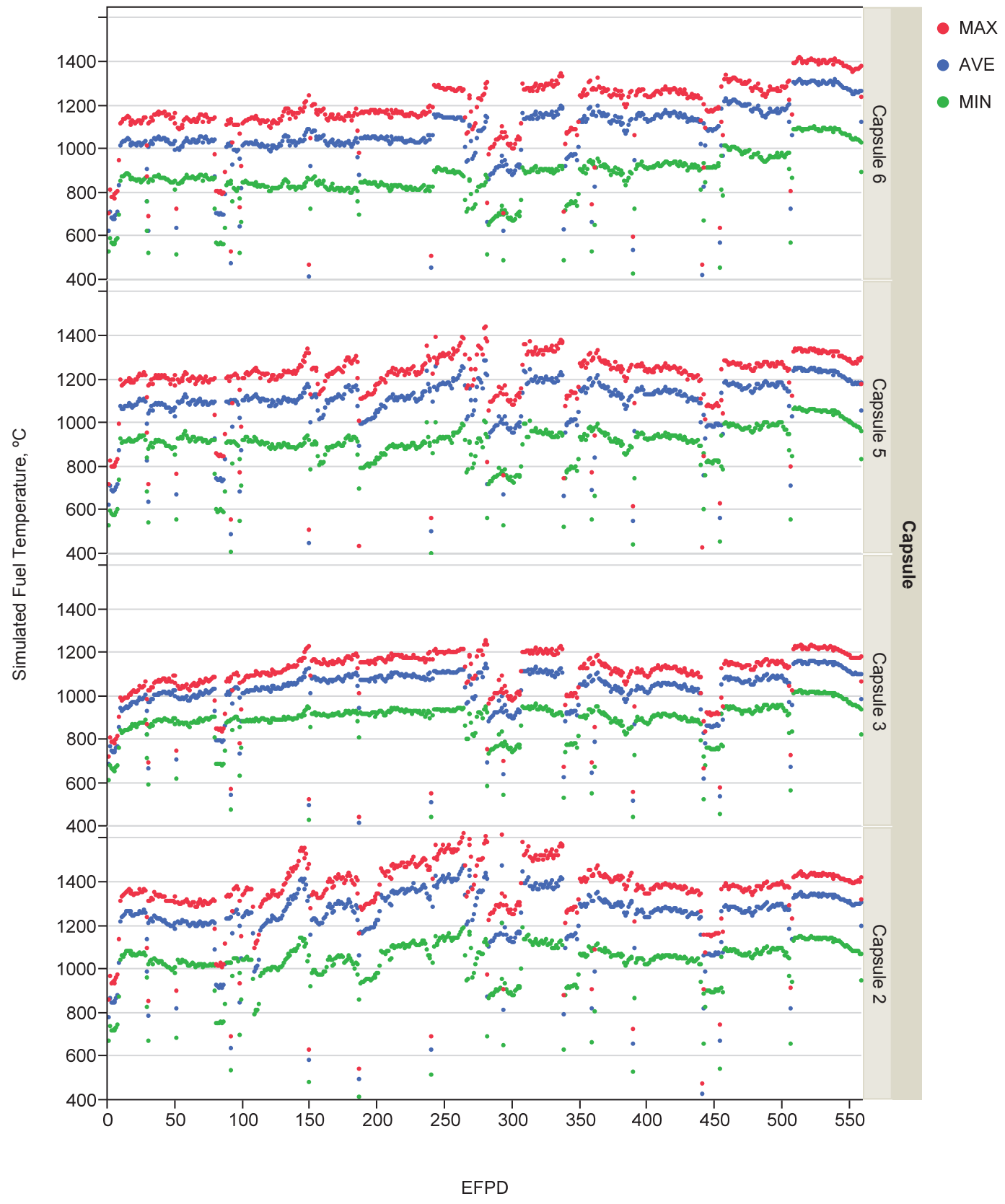

Figure 7. Calculated temperature history of daily minimum, maximum, and volume average for AGR-2 Capsules 6, 5, 3, and 2. 


\section{UNCERTAINTY QUANTIFICATION OF MODEL PREDICTED TEMPERATURE}

\subsection{Approach}

\subsubsection{Overall Uncertainty of AGR-2 Predicted Temperatures}

In general, uncertainty in the prediction of a simulation model arises from two main sources: input uncertainty and model uncertainty. This is assuming that the numerical errors can be eliminated by the use of high resolution computing code. Subsequently, the overall uncertainty of simulation model predictions in terms of variance can be expressed as:

$\sigma_{T}^{2}=\sigma_{P}^{2}+\sigma_{B}^{2}$

where

$\sigma_{T}^{2}$ is the overall uncertainty of predicted temperature in terms of variance

$\sigma_{P}^{2}$ is the input uncertainty in terms of variance

$\sigma_{B}^{2}$ is the model bias in terms of variance.

\subsubsection{Input Uncertainty}

Input uncertainty refers to incomplete knowledge of correct values of model inputs, which exists independently with any model, but will impact the uncertainty of model prediction. To quantify the input uncertainty of AGR calculated temperatures, ABAQUS model input parameters of potential importance are identified. Identification has two parts: (1) using expert judgment, determine parameters with the largest uncertainties and estimate these uncertainties, and (2) using sensitivity analysis, determine parameters that the modeling is most sensitive to, and refine the estimates of these sensitivities.

The parameter uncertainties and sensitivity coefficients are combined and propagated to quantify the input uncertainty using Equation (4), because the predicted temperature can be assumed to be the weighted summation of input parameters. This assumption is confirmed during sensitivity analysis.

$\sigma_{P}^{2}=\sum_{i}^{n} a_{i}^{2} \sigma_{i}^{2}+\sum_{i}^{n} \sum_{j \neq i}^{n} \rho_{i j} a_{i}^{2} \sigma_{i}^{2} a_{j}^{2} \sigma_{j}^{2}$

where

$a_{i}^{2}$ is the square of the sensitivity coefficient for parameter $i$

$\sigma_{i}^{2}$ is the uncertainty of input parameter $i$ in terms of variance

$\rho_{i j}$ is the correlation coefficient for input parameters $i$ and $j$.

\subsubsection{Thermal Model Uncertainty}

Model uncertainty usually arises from assumptions associated with the mathematical form or structure of the model. This section discusses the rationales of negligible model uncertainty of AGR-2 calculated temperatures. 


\subsubsection{Variable Gas Gap Improves Model Uncertainty}

Early analysis of thermocouple data for the AGR-2 experiment as well as for AGR-1 indicated that they performed reliably, especially during the beginning cycles of irradiation. Therefore, the AGR-2 thermal models also were calibrated to match the TC readings during this portion of the irradiation. Because AGR-2 capsules are similar to AGR-1 capsules, the rationale for basis of uncertainty analysis for AGR-2 thermal models are partly based on data from AGR-1 capsules.

For the AGR-1 experiment, the updated control gap distance models for all capsules led to significantly improved fit between calculated and measured TC readings (Pham et al. 2014). Figure 8 presents temperature residuals (differences between measured and calculated temperatures) of two peripheral TCs in AGR-1 Capsule 4 (top row) and Capsule 6 (bottom row) for two versions of the AGR thermal model as functions of EFPD. The only difference between the two versions of the thermal model is the gas gap distance model: the first version (run 1) assumes constant gas gap over time and the second version (run 2) assumes linear gap change depending on fluence. The peripheral TCs are used for demonstration of improved fit because temperature at these TCs is most sensitive to variation of the gap distance. In other words, the updated gas gap model has the most effect on predicted temperatures at these TC locations. Capsules 4 and 6 are used because of their stark differences of boron carbide concentration ( $7 \%$ B4C versus $5.5 \%$ B4C) and location in the test train (middle vs. top). These differences lead to different directions of the gap change: the gap in Capsule 4 is decreasing significantly (up to $50 \%$ of start gap) because of swelling in the graphite holder while the gap in Capsule 6 is slightly increasing (up to $\sim 10 \%$ ) due to shrinkage in the graphite holder.

As a result, TC residuals in Capsule 4 (top row in Figure 8) show much improvement in terms of TC fitting for run 2 results (dot symbols show a random pattern around zero for the entire experiment) relative to run 1 results (triangle symbols show downward trend over time indicating clear departure of TC readings from simulation as the experiment progresses). The continued agreement of these TC readings over the entire AGR-1 experiment suggests negligible model bias of the run 2 results with updated linear gas model. This leads to reduction of overall temperature prediction uncertainty due to elimination of the model bias. The improvement in agreement between measured and calculated TCs in Capsule 6 is much smaller (slightly flatter run 2 TC residuals as shown by the dots in the bottom row in Figure 8) due to much smaller gap change over time. 

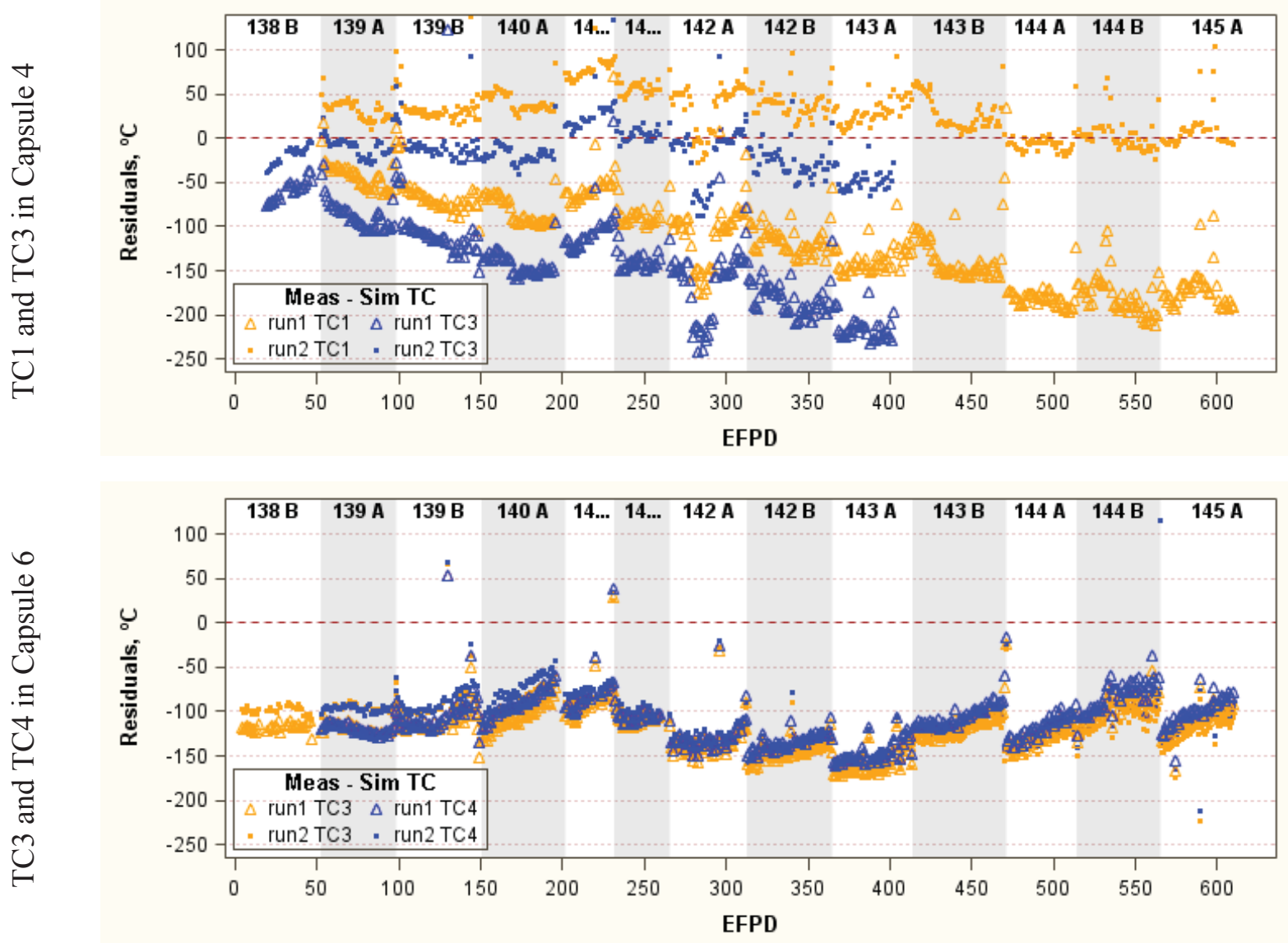

Figure 8. The thermocouple residuals of the original (run 1) and updated (run 2) thermal models for AGR-1 Capsules 4 and 6.

For the AGR-2 experiment, the weight percent boron $\left(\% \mathrm{~B}_{4} \mathrm{C}\right)$ present in the graphite material is $4.83 \%$ in Capsule 6, 4.95\% in Capsule 3, and 5.75\% in Capsules 5 and 2. Thus, the boron contents for AGR-2 capsules are similar to AGR-1 Capsule 6 (5.5\% boron content), therefore the graphite holders are assumed to shrink at similar rate as for Capsule 6. This graphite holder shrinkage leads to slightly wider control gas gap between holder and capsule body (10\% to $20 \%$ gap increase) as the irradiation progresses. As a result, the updated variable control gap distance models for AGR-2 capsules leads to small increase in predicted TC temperatures over time. The residuals for operational TCs in the AGR-2 U.S capsules are plotted in Figure 9. Generally, the residuals beyond the calibration period showing the same pattern for all TCs up to their failure dates increase the confidence in the assumed gas gap variation models. The TC residuals for TC3 in Capsule 6 (green dots in Figure 9) are exceeding $200^{\circ} \mathrm{C}$ indicating the drift failure and its readings are "Trend" data as reported in (Pham and Einerson 2014). 


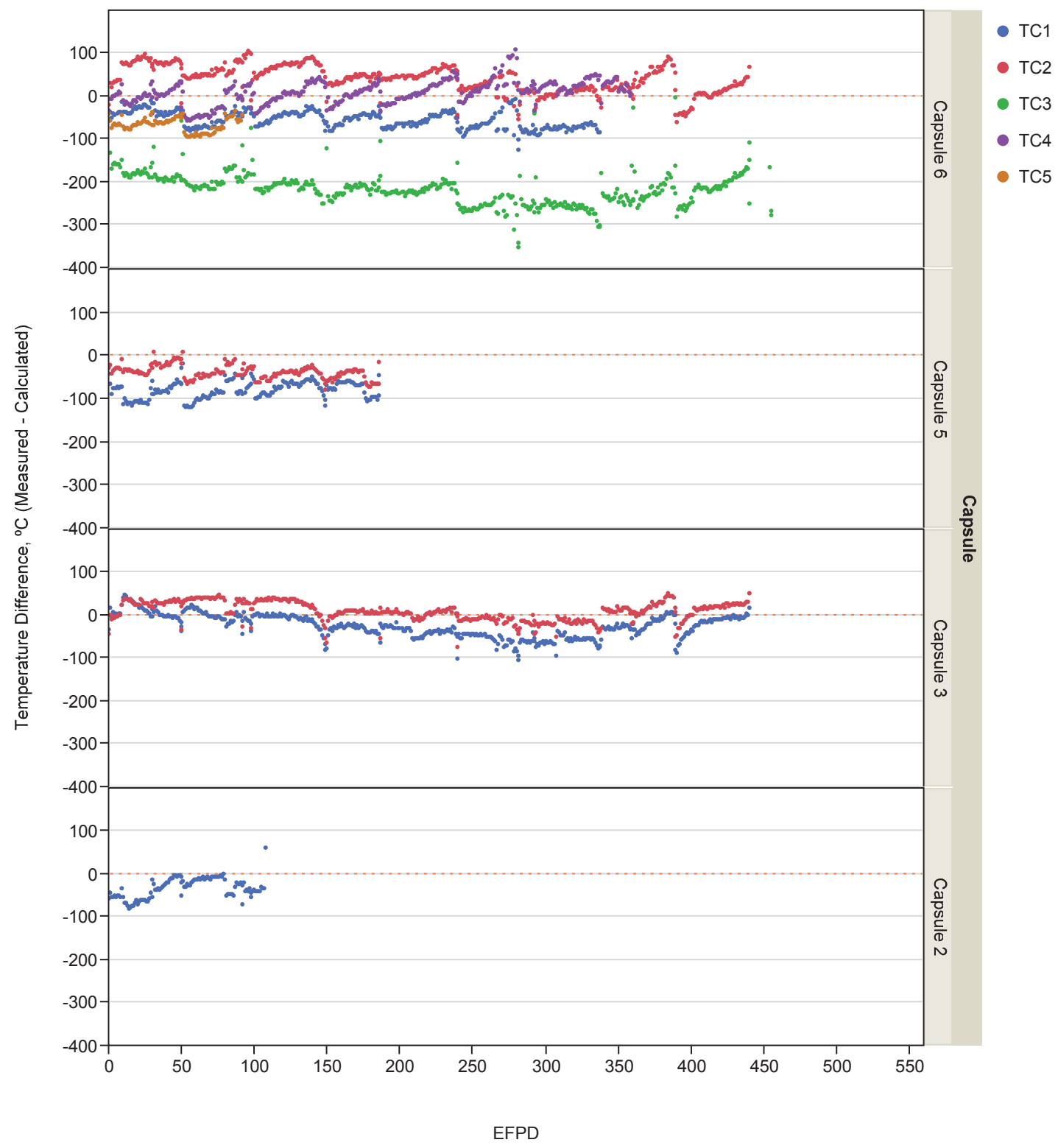

Figure 9. Difference between measured and predicted thermocouple temperatures for AGR-2 U.S. capsules.

\subsubsection{Thermal Model Bias}

The histograms of daily residuals for TC1 and TC2 in each of four U.S. capsules, and their statistics (average and standard deviation), are presented in Figure 10. For Capsule 3 and Capsule 6 (excluding TC3 as seen in the top panel of Figure 9) TC residuals are on both sides of zero, indicating that the model bias is negligible for these two capsules. For Capsule 2 and Capsule 5 TC residuals are generally negative indicating that the thermal models are likely over predicting temperature for Capsules 2 and 5. For Capsule 2, there is only one operating TC for two ATR cycles, so the model bias is not conclusive. By contrast, Capsule $5 \mathrm{TC}$ residuals are consistently less than 0 for both $\mathrm{TCs}$ (i.e., $-82^{\circ} \mathrm{C}$ for $\mathrm{TC} 1$ and $-40^{\circ} \mathrm{C}$ for TC2); therefore the model bias of $60^{\circ} \mathrm{C}$ is likely for Capsule 5 calculated temperatures, assuming TC1 and TC2 have not drifted. However, it is assumed that there is insignificant model uncertainty for all capsule temperatures with precaution of potential model uncertainty in Capsule 5. 


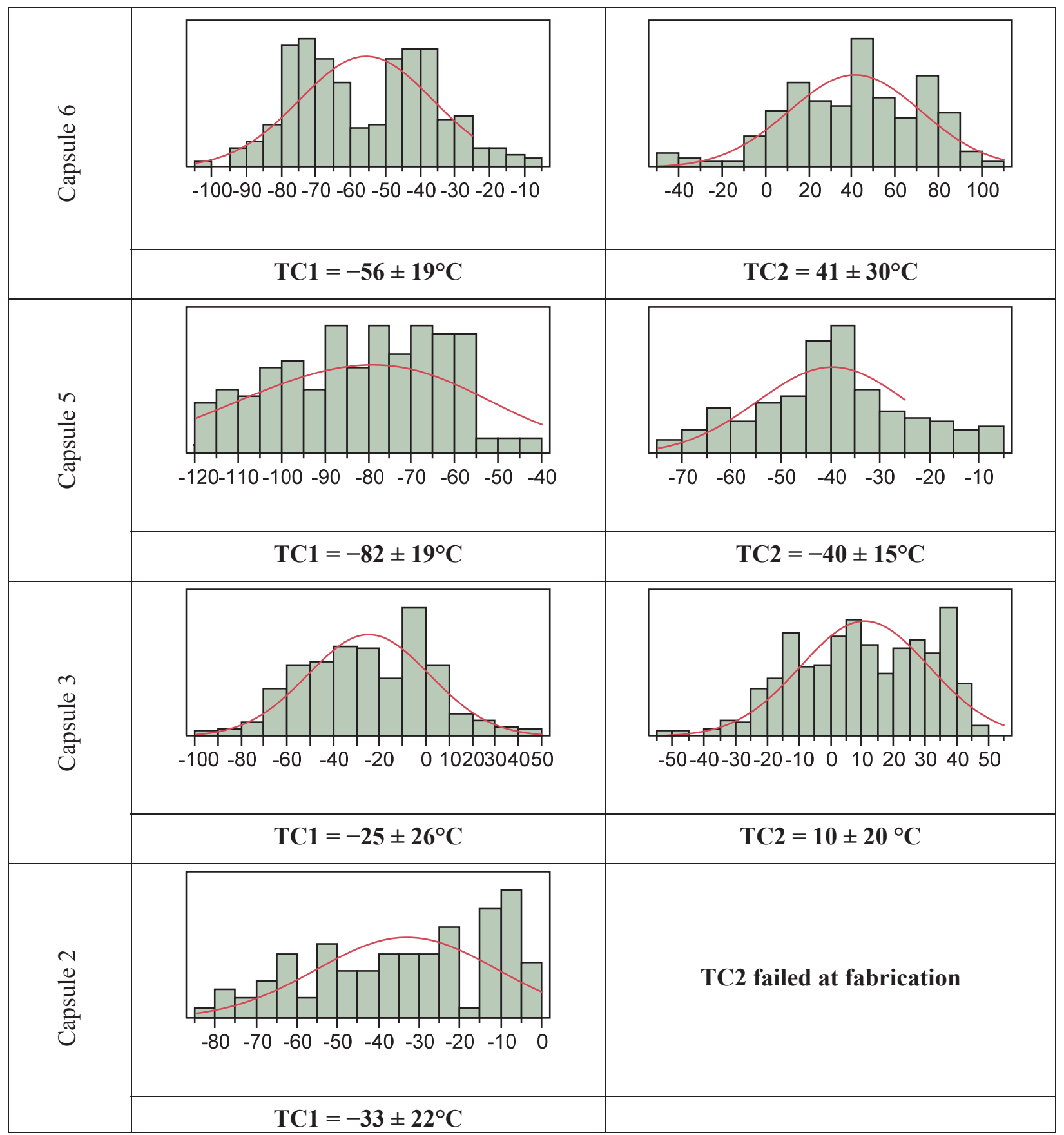

Figure 10. Histograms of temperature residuals of peripheral TCs of four U.S. capsules.

\subsection{Influential Input Parameter Selection}

The selection of input parameters for uncertainty quantification of the AGR-2 calculated temperatures is based on the ranking of their influences on variation of temperature predictions. The influence ranking is highest for parameters with large uncertainty and/or large sensitivity. The sensitivity evaluation of the temperature calculations was performed for the AGR-2 experiment on an individual capsule (Capsule 5) by the modeler ${ }^{\mathrm{a}}$. A series of cases was compared to a base case by varying different input parameters to

${ }^{a}$ Gant L. Hawkes e-mail to Binh T. Pham, November 13, 2014. 
the ABAQUS finite element thermal model for Capsule 5 at time step 43 of ATR Cycle 147A. The tornado plots in Figure 11 shows the most sensitive input parameters on peak fuel temperature (left) and volume-average (VA) fuel temperature (right), sorted from largest to smallest for peak fuel temperature. Figure 12 shows parameter sensitivities for TC1 temperature. Apparently, the most sensitive parameters for both fuel and TC temperatures are heat rate in the fuel, control gas composition (e.g., neon fraction), and control gap distance. The next four are heat rate in the graphite, graphite thermal conductivity, fuel conductivity, and gap conductivity between compact and graphite holder. These parameters have different impacts on fuel and TC temperatures. The fuel conductivity has much more influence on peak fuel temperatures, while the heat rate in graphite has bigger impact on $\mathrm{TC} 1$ temperature.

Table 1 presents the range of input uncertainties for the five most uncertain input parameters as judged by the ART AGR experts (Petti, et al. 2014). Expert judgment takes into account machining tolerances for capsule geometry, measurement uncertainty of mass flow controllers, model uncertainty of neutronics analysis results, and legacy experience for fuel compact and graphite conductivity.

After combining input parameter uncertainties with sensitivities, the five most influential input parameters on AGR-2 calculated temperatures are control gas gap distance, heat rate in fuel compacts, neon fraction, graphite thermal conductivity, and fuel compact thermal conductivity. The first three parameters were chosen because of high sensitivity and the last two, having much lower sensitivity, were chosen because of their significantly higher input uncertainty.

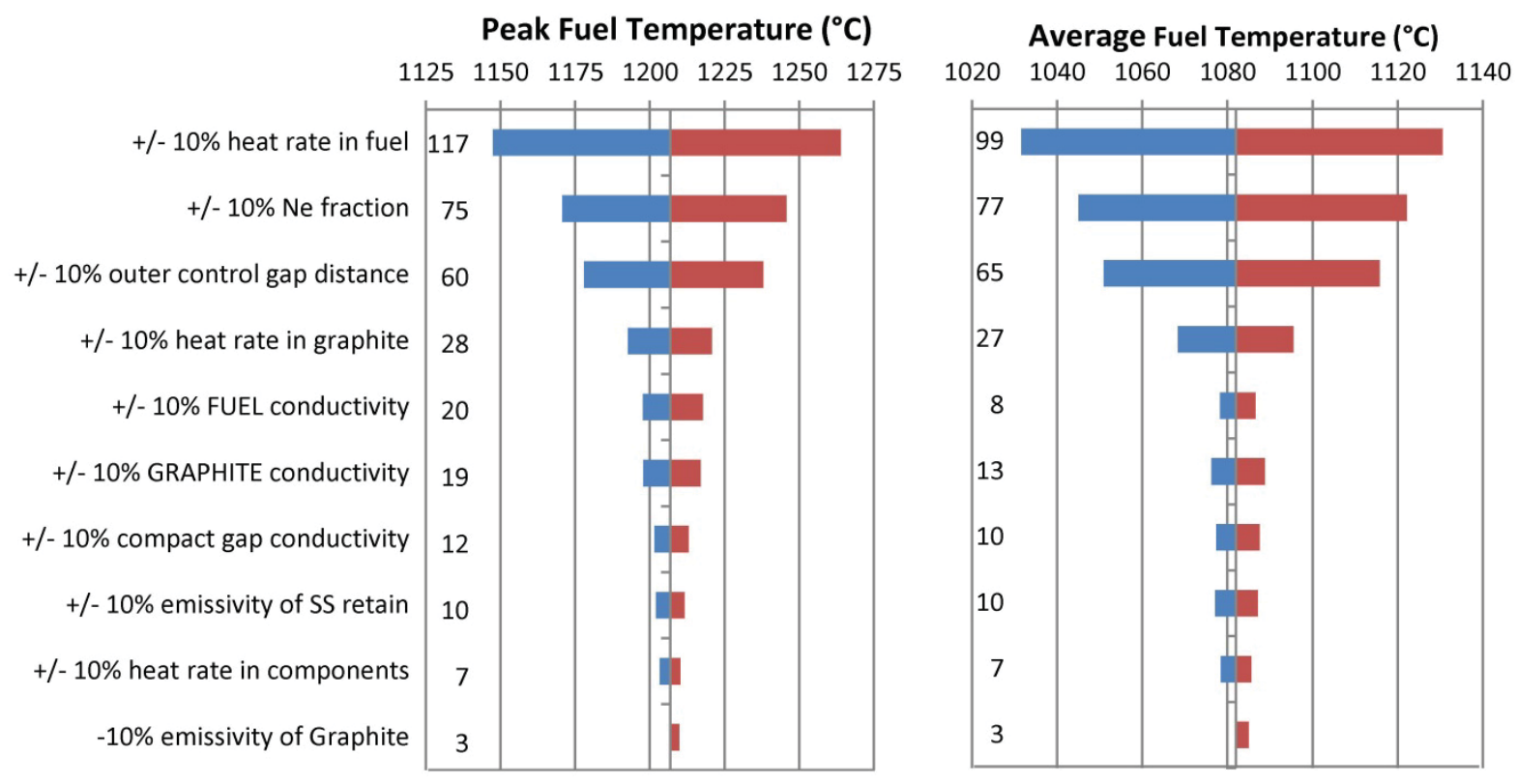

Figure 11. Parameter sensitivities for Capsule 5 peak and VA fuel temperatures. 


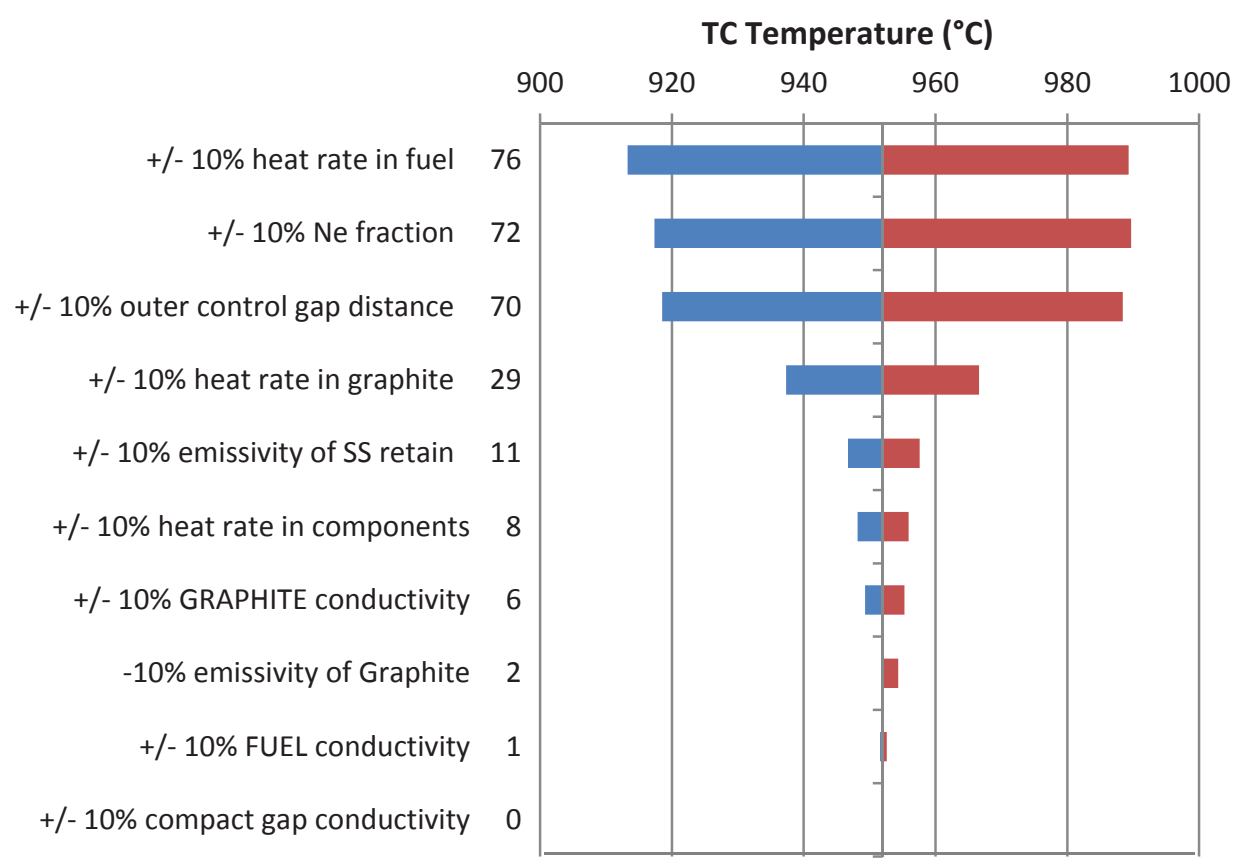

Figure 12. Parameter sensitivities for Capsule 5 calculated TC temperature.

Table 1. Uncertainties of the most significant parameters of the AGR-2 thermal model.

\begin{tabular}{|c|c|c|}
\hline Parameter & Uncertainty (\%) & Rationale \\
\hline $\begin{array}{l}\text { Control gap } \\
\text { distance }\end{array}$ & $\begin{array}{l}\text { Function of fast fluence: } \\
\text { Capsule 6: } 4.3-6.5 \\
\text { Capsule 5: } 6.5-9.7 \\
\text { Capsule } 3: 3.2-4.8 \\
\text { Capsule 2: } 3.7-5.5\end{array}$ & $\begin{array}{l}\text { Uncertainty at start of irradiation is } 1 \text { mil based on fabrication } \\
\text { tolerance. } \\
\text { Uncertainty over the course of irradiation is time dependent } \\
\text { because of AGR-2 graphite holder shrinkage. The graphite } \\
\text { shrinkage is proportional to the reaction rate in the graphite } \\
\text { leading to a physics-based linear gas gap model similar to } \\
\text { AGR-1 Capsule } 6 \text {, which has similar boron content. Thus, the } \\
\text { gap uncertainty is assumed to be a linear function of fluence } \\
\text { and is increased to } 150 \% \text { of initial uncertainty by the end of } \\
\text { irradiation (Subsection 3.3.1). }\end{array}$ \\
\hline Neon fraction & $\begin{array}{l}\text { Function of neon } \\
\text { fraction }\end{array}$ & $\begin{array}{l}\text { For the time period before cross-talk and during uniform gas } \\
\text { mixture, uncertainty is based on } 1 \mathrm{sccm} \text { flow rate tolerance. } \\
\text { For the time period between cross-talk start and uniform gas } \\
\text { mixture start, uncertainty is estimated from neon fraction } \\
\text { prediction equation. }\end{array}$ \\
\hline Fuel heat rate & 5 & $\begin{array}{l}\text { Basis is AGR-1 comparison done by J. Harp with additional } \\
\text { input from J. Sterbentz (Subsection 3.3.3). }\end{array}$ \\
\hline $\begin{array}{l}\text { Graphite } \\
\text { conductivity }\end{array}$ & 15 & $\begin{array}{l}\text { Additional conductivity data for the test graphite allows a } \\
\text { lower uncertainty estimate for graphite than for fuel. }\end{array}$ \\
\hline $\begin{array}{l}\text { Fuel } \\
\text { conductivity }\end{array}$ & 20 & $\begin{array}{l}\text { Uncertainty is based on work done on surrogate compacts } \\
\text { by C. Folsom at Utah State university. }\end{array}$ \\
\hline
\end{tabular}




\subsection{Input Parameter Uncertainties}

Table 1 presents the range of input uncertainties for the five most uncertain input parameters as judged by the ART AGR experts. However, the input uncertainties over the course of irradiation are dynamic, accounting for the effect of unplanned events (e.g., the gas line cross-talk failure) and changes in thermal properties of capsule components (e.g., assumed increase in control gas gap distance) over extended exposure to high temperatures and fast neutron irradiation. This section lays out the basis for determination of input uncertainty of five selected parameters for each time step over the entire AGR-2 irradiation.

\subsubsection{Control Gap Distance}

At the beginning of irradiation the as-fabricated graphite holder outer diameter and capsule retainer sleeve inner diameter are adjusted, taking into account the thermal expansions when capsules are brought up to temperature. The "hot" control gap distance, equal to a half of the difference between the above two adjusted diameters, is used in the ABAQUS model to predict temperatures in each capsule. These values are called initial control gaps, and are presented in the second column of Table 2. At this point in time, the uncertainty of control gap distance was based on machining tolerance and assumed to be about one-thousandth of an inch (1 mil) for all six capsules.

As the experiment progresses, the material properties of capsule components are changing because of high temperatures and neutron fluence. For the AGR-1 experiment, the dimensional measurements of the compacts, graphite holders, and steel capsule shells were performed during PIE and reported in (Demkowicz 2011). The results show that the control gap shrank for the four middle Capsules (2-5) with $7 \% \mathrm{~B}_{4} \mathrm{C}$ and expanded for the top and bottom Capsules (1 and 6) with 5.5\% $\mathrm{B}_{4} \mathrm{C}$. Because the properties of AGR-2 compacts and the boron content of graphite holder are similar to AGR-1 Capsule 6, the AGR-2 gas gap change model is assumed to be the same as for AGR-1 Capsule 6. Explicitly, the holder holes show a shrinkage rate of $-0.23 \%$ per $1 \times 10^{25} \mathrm{n} / \mathrm{m}^{2}$, while the holder outside diameter is at $-0.18 \%$. Thus, the control gap distance for day i $\left(\Delta x_{i}\right)$ can be calculated as:

$$
\Delta x_{i}=\Delta x_{s}+0.0018 * r * \text { fluence }_{i}
$$

where $\Delta x_{s}$ is start gap distance, $r$ is the radius of graphite holder, and is cumulative fluence in $\left(10^{25} \mathrm{n} / \mathrm{m}^{2}\right)$ on day $(i)$.

Figure 13 shows the initial and end control gap distances with their associated uncertainties for four U.S. capsules. The initial (start) gap distances (blue bars) have 1 mil error bars due to fabrication tolerance. For the gap uncertainty at the end of irradiation, the assumption of the AGR-2 gap variation rate Equation (5) using PIE data of the similar AGR-1 Capsule 6 would lead to additional gap uncertainty over time. However, this control gap model is well justified by the flat TC temperature residuals as function of EFPD of irradiation as shown in Figure 9 for all TCs. Therefore, the gap distance uncertainty at the end of irradiation (red bars) can be assumed to increase to 1.5 mil (or 1.5 times of the initial gap uncertainty). This assumption leads to an increase of the relative gap uncertainties as shown by the last columns in Table 2. For each day during irradiation, because the gas gap distance is increasing proportionally with cumulative fluence, it is also reasonable to assume that the gap uncertainty is linearly increasing with cumulative fluence as follows:

$$
\sigma_{G G_{i}}=\sigma_{G G_{S}}+\frac{\text { fluence }_{i}}{\text { fluence }_{e}}\left(\sigma_{G G_{e}}-\sigma_{G G_{s}}\right)
$$

where is the gas gap uncertainty on day $(i)$; and are the control gas gap uncertainty at the start and end of irradiation assuming that $=1.5 *$; and are cumulative fluence in $\left(10^{25} \mathrm{n} / \mathrm{m}^{2}\right)$ up to day $(i)$ and at the end of irradiation. 
Table 2. Control gap distance variation and uncertainty for AGR-2 capsules.

\begin{tabular}{|c|c|c|c|c|c|}
\hline Capsule & $\begin{array}{c}\text { Initial Gap } \\
\text { (in.) }\end{array}$ & $\begin{array}{c}\text { End Gap } \\
\text { (in.) }\end{array}$ & \% Gap Change & $\begin{array}{c}\text { Fabrication } \\
\text { Error } \\
(\sigma)\end{array}$ & $\begin{array}{c}\text { End of } \\
\text { Irradiation Error }\end{array}$ \\
\hline Capsule 6 & 0.023 & 0.0256 & $11.3 \%$ & $4.3 \%$ & $6.5 \%$ \\
\hline Capsule 5 & 0.0155 & 0.0190 & $22.6 \%$ & $6.5 \%$ & $9.7 \%$ \\
\hline Capsule 3 & 0.031 & 0.0346 & $11.6 \%$ & $3.2 \%$ & $4.8 \%$ \\
\hline Capsule 2 & 0.0273 & 0.0308 & $13.0 \%$ & $3.7 \%$ & $5.5 \%$ \\
\hline
\end{tabular}

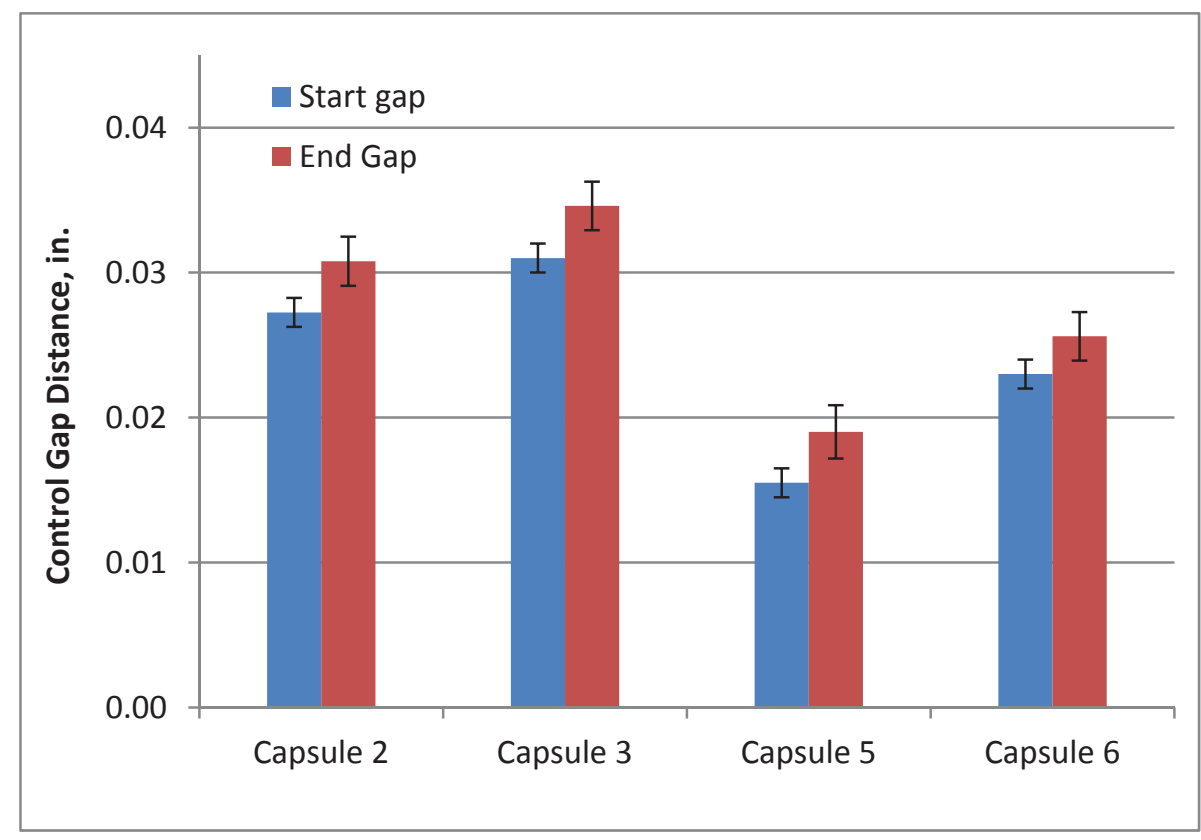

Figure 13. The initial and end gap distance with an error bar of 1 mil for four U.S. capsules.

\subsubsection{Neon Fraction}

Heat produced in the fuel compacts and graphite holder is transferred through the control gas gaps via a gap conductance model using the gap width and the conductivity of the sweep gas (Hawkes 2014). The neon fraction of the capsule control gas mixture is, in turn, used in the kinetic theory of gases to determine the gas mixture conductivity. As designed, the capsule gas lines do not cross-talk with each other before gas enters an individual capsule, and the amount of gas leaking out from a capsule gas line to the leadout is negligible. Therefore, the extra gas leaking in to a capsule is assumed to come only from the leadout flow. As a result, the calculation formula for neon fraction in each capsule, including potential gas leakage both to and from the leadout flow and when the leadout flow contains any amount of helium and neon, is expressed as

$$
F r_{N e}=\frac{Q_{N e}+\max \left(Q_{\text {outlet }}-Q_{N e+H e}, 0\right) * \frac{Q_{\text {Neleadout }}}{Q_{\text {Ne+Heleadout }}}}{\max \left(Q_{\text {outlet }}, Q_{N e+H e}\right)}
$$


where is the neon fraction and Q is the gas flow in $\mathrm{sccm}$. The neon fraction is 0 for all outage periods. The main source of neon fraction uncertainty is the measurement error in the gas flow meters, which have a $1 \mathrm{sccm}$ tolerance (Table 1) based on engineering assessment. A neon fraction simulation of Equation (7), with neon and helium flows taken randomly from a normal distribution with the mean value and a standard deviation of $1 \mathrm{sccm}$, was performed for different neon fraction levels. The neon fraction uncertainty for each neon fraction level is equal to the standard deviation calculated from 100,000 random neon fraction results. Figure 14 plots the relative neon fraction standard deviations (uncertainty) as a function of the neon fraction values. The power equation option of the trend line feature of the Microsoft Excel platform is used to estimate a function that results in a good fit to the data $\left(R^{2}=0.9924\right)$. Therefore the relative neon fraction uncertainty can be expressed as a function of neon fraction as

$$
\sigma_{F r_{n e}}=\frac{2.5487}{F r_{N e}{ }^{1.047}}
$$

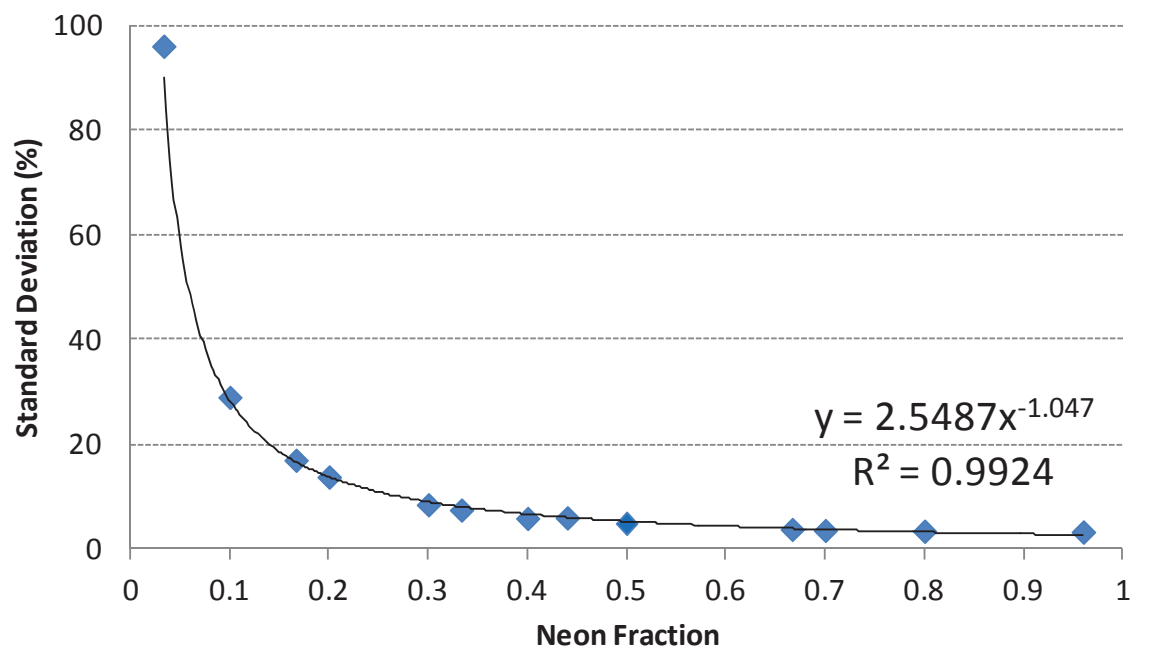

Figure 14. Neon fraction uncertainty and the trend line.

Neon fraction uncertainty is estimated well using a power function of neon fraction. Thus the relative uncertainty is high at low neon fraction ( $\sim 100 \%$ at 0.03 neon fraction) because of high relative uncertainty of the mass flow controllers at lower neon flow rates. However, when AGR-2 runs on pure helium (i.e., capsule neon flow rate is $0 \mathrm{sccm}$ ), it becomes certain that $F R_{N e} \approx 0$, resulting in a drop in neon fraction uncertainty to about $3 \%( \pm 1 / 30)$ due to $1 \mathrm{sccm}$ uncertainty of neon flow rate. Conversely, when the capsule and leadout gas flows are pure neon (i.e., capsule neon flow rate is $30 \mathrm{sccm}$ ), then it is also certain that $F R_{N e} \approx 1$, but the neon fraction uncertainty is near $0 \%[\sim 1 /(30 * 30)]$ due to lower relative uncertainty of neon flow rate $(1 / 30)$.

The assumption that the capsule gas lines do not cross-talk with each other before gas enters an individual capsule is violated beginning with ATR Cycle 150B, soon after the AGR-2 was reinserted into the ATR core (Pham 2014). The gas line cross-talk failure allows the gas mixture from one capsule to enter other capsules; therefore Equation (6) cannot be used to calculate neon fraction for each capsule when their gas mixtures are different. However, this cross-talk failure also impacts neon fraction calculation only during ATR Cycle 150B because after Cycle 150B, ART program management decided to use uniform neon and helium gas mixtures for all six capsules as well as the leadout. This operating procedure was fully implemented on January 17, 2012, in the middle of Cycle 151A (following a pure helium gas flow during the beginning of this cycle), as shown in Figure 15. This uniform gas mixture in all capsules and leadout allows the neon fraction calculation using Equation (7) and the neon fraction uncertainty can be determined using Equation (8). 
For ATR Cycle 150B (240 to 280 EFPDs), the neon fractions were estimated as a regression function of TC readings, fuel fission power, fast fluence, outer shim control cylinder position, and cycle run time. The uncertainty of these predicted neon fractions can be relatively low because of the good fit between actual and predicted neon fractions during subsequent cycles, 151A and 151B, when capsule neon fractions can be accurately calculated (TEV-2004). As a conservative estimate, neon fraction uncertainty during ATR Cycle 150B is assumed to be double the normal neon fraction uncertainty.

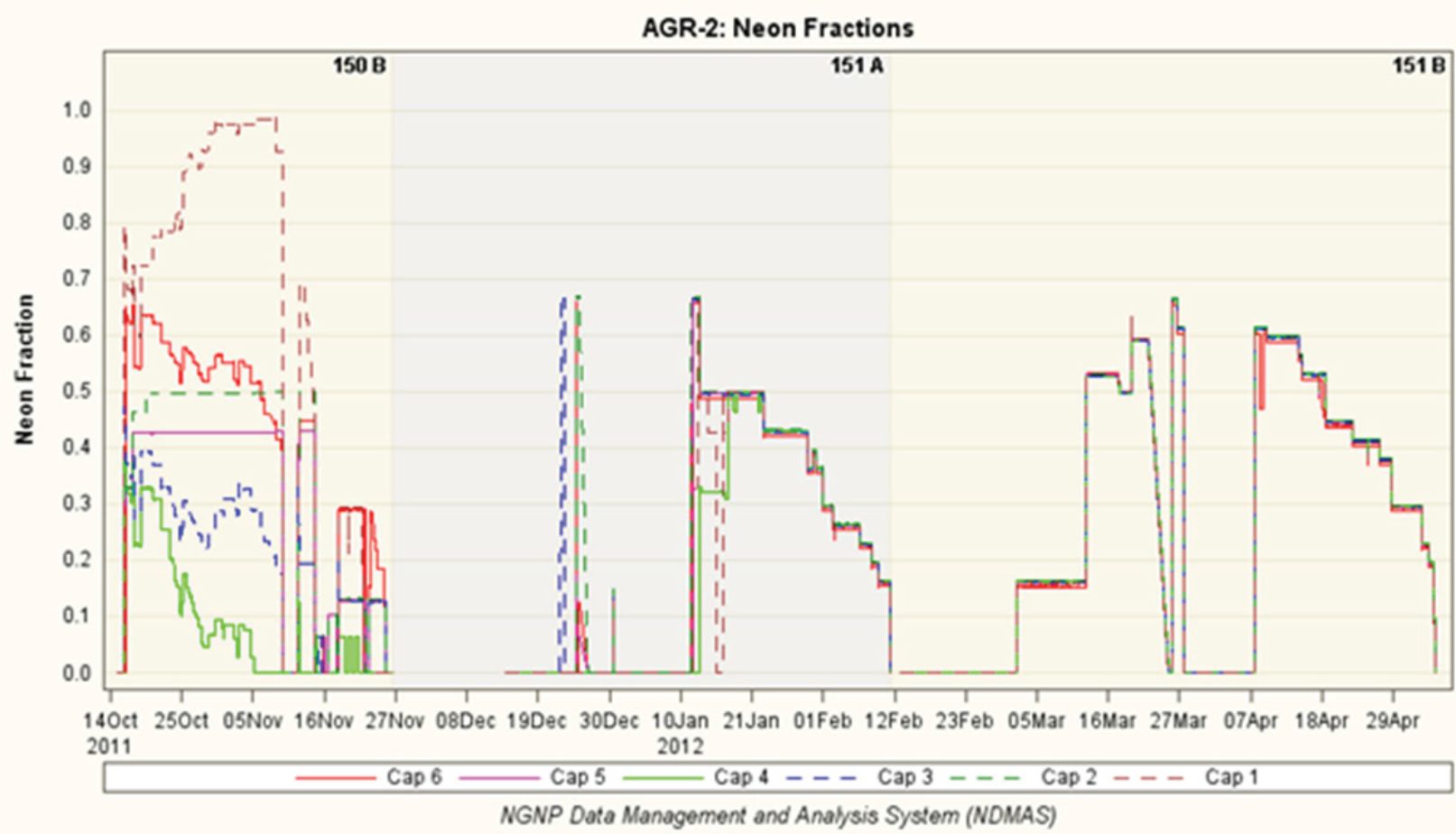

Figure 15. Capsule neon fractions after 150A PALM cycle. The neon fractions for all capsules were set to the same value beginning January 17, 2012 (ATR Cycle 151A).

\subsubsection{Fuel Heat Rate}

The fuel heat rates are taken from the as-run physics calculation (ECAR-2066). Figure 16 shows the power density averaged for each capsule versus the time of irradiation in EFPDs. The fuel compacts reach their peak heat rates for all capsules about halfway through the irradiation (280 EFPDs). The uncertainty in the calculated fuel heat rate is a collection of several factors from ATR measured data input parameters that go into the physics calculation and Monte Carlo statistical uncertainties associated with calculated parameters. These specific uncertainties include:

1. ATR total core or lobe power of $\pm 4.1 \%$

2. Fuel compact uranium begin-of-life number densities of $\pm 0.5 \%$

3. Calculated irradiation flux of $\pm 1.0 \%$

4. Calculated reaction rates or a one group cross section of $\pm 2.0 \%$

5. Power normalization factors of $\pm 1.0 \%$

6. Outer shim control cylinder hafnium and beryllium reflector poison number densities of $\pm 1.0 \%$

7. Outer shim control cylinder rotational position of $\pm 0.5 \%$.

Assuming these individual uncertainties to be random, the overall fuel heat rate uncertainty can be estimated to be $\pm 5.0 \%$ for all capsules and all cycles, including the PALM cycle, when AGR-2 test was 
moved to the I-24 location. In addition, good agreement between burnup calculated by the physics depletion model and PIE measurements for AGR-1 experiment, where the difference is less than 10\% for the worst compact, indicates that the instantaneous (daily) fuel fission power uncertainty should be small.

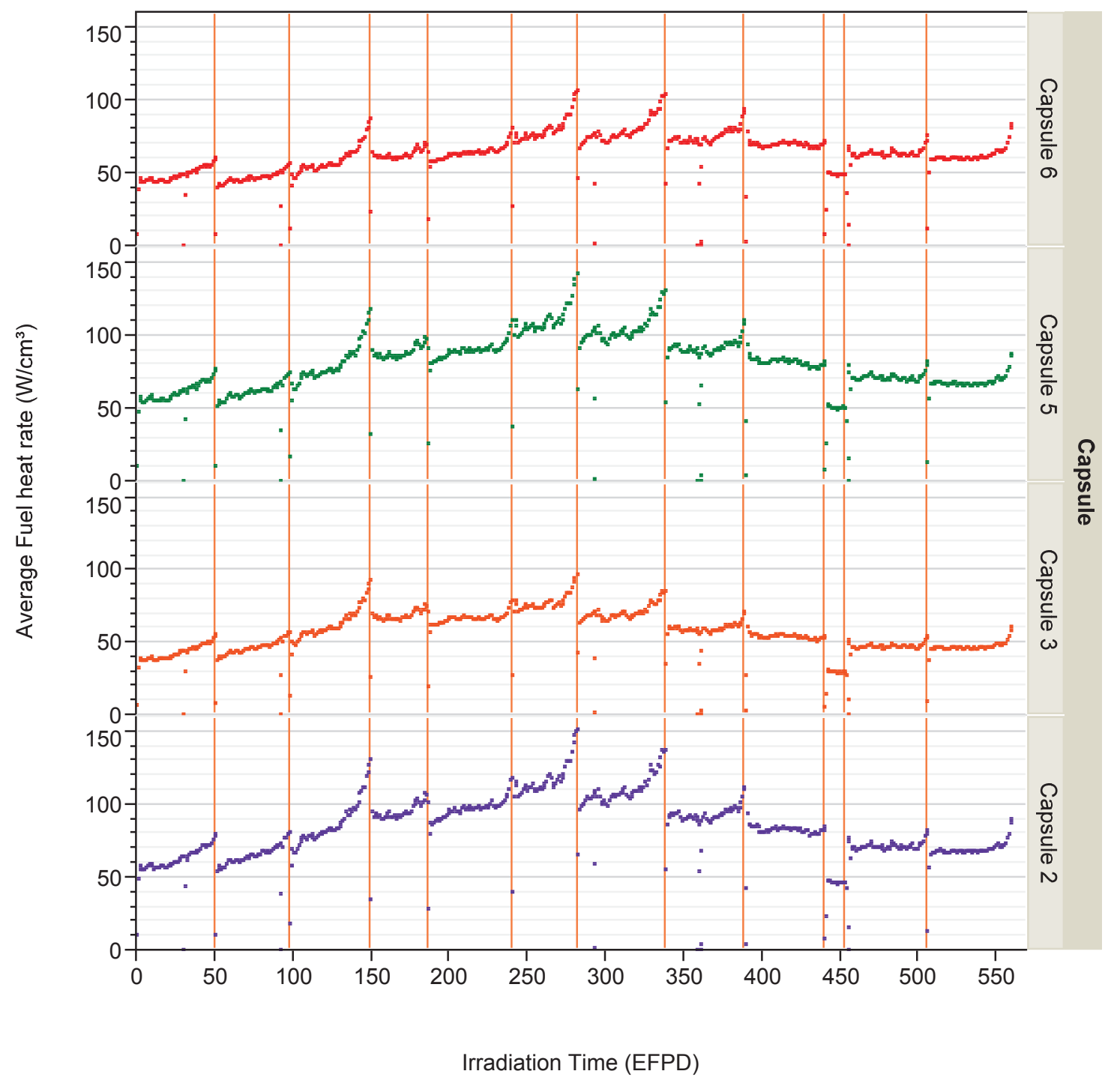

Figure 16. Capsule average power density versus irradiation time in EFPD.

\subsubsection{Graphite Thermal Conductivity}

Unirradiated graphite thermal conductivity data for the holders were provided by GrafTech as a function of temperature and the weight percent boron carbide present in the material (Snead and Burchell 1995). The effect of irradiation on the thermal conductivity of the graphite was accounted for in this analysis using the correlation

$$
\begin{aligned}
& \frac{k_{i r r}}{k_{0}}=\left(0.25-0.00017 * T_{i r r}\right) * A^{*} \log (d p a)+0.000683 * T_{i r} \\
& A=-1.0
\end{aligned}
$$


where $k_{i r r}$ and $k_{0}$ are thermal conductivities of irradiated and unirradiated graphite, respectively, $T_{i r r}$ is the irradiation temperature $\left({ }^{\circ} \mathrm{C}\right)$, and $d p a$ is displacements per atom. The multiplier used to convert fast fluence $(>0.18 \mathrm{MeV})$ to dpa is $8.23 \times 10^{-26} \mathrm{dpa} /\left(\mathrm{n} / \mathrm{m}^{2}\right)$. Figure 17 shows a three-dimensional plot of this ratio $\left(k_{i r} / k_{o}\right)$ varying with dpa and temperature. The ratio of irradiated to unirradiated thermal conductivity is increasing with higher temperatures and decreasing with higher dpa (or fast neutron irradiation). These correlations are obtained based on different graphite properties than the graphite employed in the AGR-2 test train. The fact that the thermal conductivity for the actual AGR-2 graphite holder has to be extrapolated from given correlations also leads to higher parameter uncertainty. According to expert assessment, the existence of one data point for validation of the correlation helps to reduce the graphite thermal conductivity uncertainty from an original value of 20 to $15 \%$ for the entire AGR-2 irradiation, as shown in Table 1.

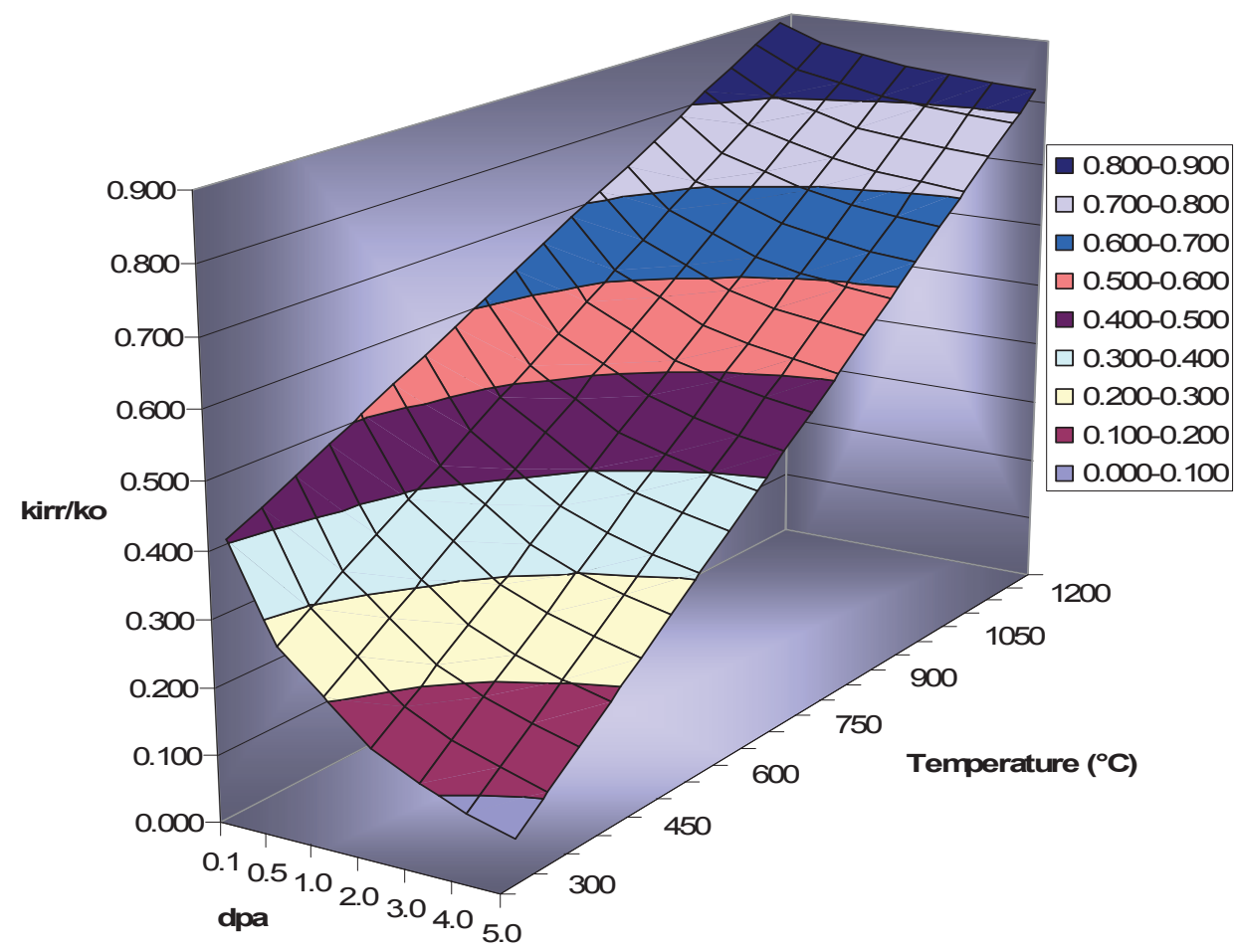

Figure 17. Ratio of irradiated over unirradiated graphite thermal conductivity $\left(\mathrm{k}_{\mathrm{irr}} / \mathrm{k}_{\mathrm{o}}\right)$ varying with temperature and dpa.

\subsubsection{Fuel Compact Thermal Conductivity}

The fuel compact thermal conductivity was taken from correlations of conductivity with temperature, temperature of heat treatment, neutron fluence, and TRISO-coated particle packing fraction (Gontard and Nabielek 1990). These correlations were further adjusted to account for differences in fuel compact density and packing fraction. The given correlations were developed for a fuel compact matrix density of $1.75 \mathrm{~g} / \mathrm{cm}^{3}$, whereas the compact matrix used in AGR-2 had a density of approximately $1.6 \mathrm{~g} / \mathrm{cm}^{3}$ for $\mathrm{UCO}$ compacts and $1.68 \mathrm{~g} / \mathrm{cm}^{3}$ for $\mathrm{UO}_{2}$ compacts. Thus the thermal conductivities for AGR-2 compacts were scaled according to the ratio of densities $\left(0.91\right.$ for $\mathrm{UCO}$ and 0.96 for $\left.\mathrm{UO}_{2}\right)$ in order to correct for this difference. Figure 18 shows a three-dimensional plot of the fuel compact thermal conductivity varying with fluence and temperature. The lack of experimental data for AGR-2 fuel compact thermal properties leads to high uncertainty of compact thermal conductivity, which is estimated to be $20 \%$ for the entire AGR-2 irradiation (Table 1). 


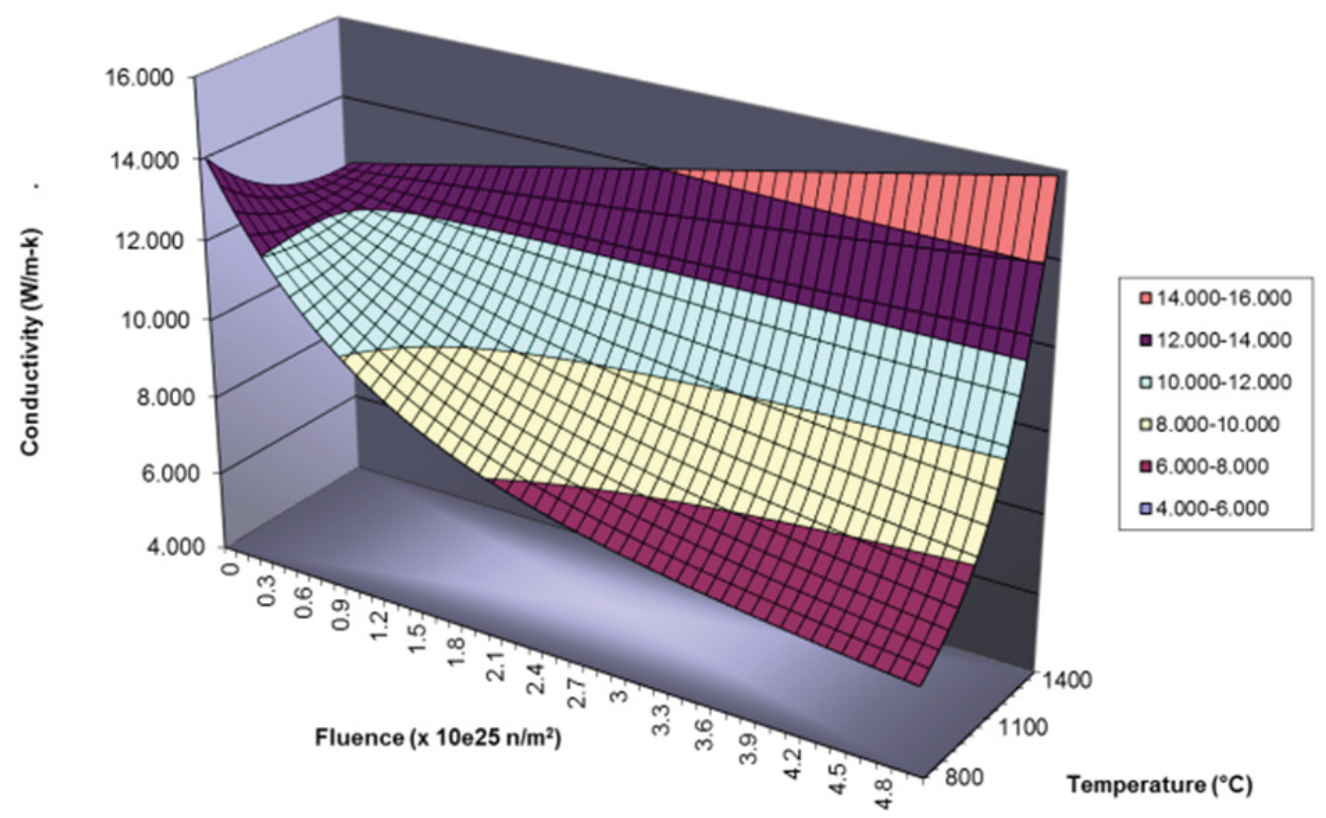

Figure 18. UCO compact thermal conductivity varying with fluence and temperature (ECAR-2476).

\subsection{Estimation of Input Parameter Sensitivity}

The governing equations for steady-state conduction and radiation heat transfer models (Equations (1) and (2)) used for AGR-2 capsule temperature calculation show complex nonlinear relationships between output temperature and input parameters over the wide variation of experimental conditions. This makes it impossible to derive a unique analytical formula to calculate output uncertainty from input variations over the whole AGR-2 experimental condition domain. Additionally, the standard Monte Carlo technique is impractical because of the necessity of requiring hundreds of thousands of simulations to estimate the overall output temperature uncertainty with satisfactory accuracy. The AGR-2 thermal model sensitivity analysis results given in Section 3.2 represent parameter sensitivities within only a small experimental condition domain of $\pm 10 \%$ of the nominal values used in the analysis. Also, the existing sensitivity analysis in (Hawkes et al. 2011) does not include the investigation of interactive effects of input variables.

\subsubsection{Statistical Experimental Design for Sensitivity Analysis}

To be computationally efficient, statistical experimental design was used to develop the set of simulation runs necessary to estimate all main effects and pairwise interactions of the five important input variables. To overcome the nonlinearity of the temperature function (e.g., Equation (1)), the AGR-2 thermal condition domain is divided into multiple smaller ranges, within which the output temperature can be estimated as a linear combination of selected input variables. Subsequently, the temperature uncertainty can be calculated from given input uncertainty using standard error propagation of the linear combination (Ostle and Mensing 1975). Capsule 5 was selected for this sensitivity analysis, which will be applied for all other AGR-2 capsules.

Table 3 lists the required 51 runs of the ABAQUS code to be completed for Scenarios 1-4 in Table 4. These scenarios cover a wide range of the thermal conditions such as fuel heat rate, neon fraction, and fast neutron fluence (subsequently, fuel compact and graphite holder conductivities). However, the gas gap sensitivity strongly depends on the gap distance and Capsule 5 has the smallest control gap (only half of the Capsule 3 gap). Thus, to determine the gas gap sensitivity for larger gap distances, three more simulation runs were performed using Capsule 3 gap distances $(0.9,1.0$, and 1.1 of the Capsule 3 gap) and Scenario 1 inputs for other parameters (Scenario 5 in Table 4). 
Table 3. Experimental design matrix for AGR-2 thermal model sensitivity analysis.

\begin{tabular}{|c|c|c|c|c|c|c|}
\hline Run & Heat Rate & Gas Gap & Ne Fraction ${ }^{b}$ & $\begin{array}{c}\text { Graphite } \\
\text { Conductivity }\end{array}$ & $\begin{array}{l}\text { Fuel compact } \\
\text { conductivity }\end{array}$ & Purpose \\
\hline 0 & Nominal $^{\text {a }}$ & Nominal & Nominal & Nominal & Nominal & Base line \\
\hline 1 & Low & Nominal & Nominal & Nominal & Nominal & \multirow[t]{2}{*}{ Estimate effect of heat rate } \\
\hline 2 & High & Nominal & Nominal & Nominal & Nominal & \\
\hline 3 & Nominal & Low & Nominal & Nominal & Nominal & \multirow[t]{2}{*}{ Estimate effect of gas gap } \\
\hline 4 & Nominal & High & Nominal & Nominal & Nominal & \\
\hline 5 & Nominal & Nominal & Low & Nominal & Nominal & \multirow[t]{2}{*}{ Estimate effect of $\mathrm{Ne}$ fraction } \\
\hline 6 & Nominal & Nominal & High & Nominal & Nominal & \\
\hline 7 & Nominal & Nominal & Nominal & Low & Nominal & \multirow{2}{*}{$\begin{array}{l}\text { Estimate effect of graphite } \\
\text { conductivity }\end{array}$} \\
\hline 8 & Nominal & Nominal & Nominal & High & Nominal & \\
\hline 9 & Nominal & Nominal & Nominal & Nominal & Low & \multirow{2}{*}{$\begin{array}{l}\text { Estimate effect of fuel compact } \\
\text { conductivity }\end{array}$} \\
\hline 10 & Nominal & Nominal & Nominal & Nominal & High & \\
\hline 11 & Low & Low & Nominal & Nominal & Nominal & \multirow{4}{*}{$\begin{array}{l}\text { Estimate interactive effect of } \\
\text { heat rate by gas gap }\end{array}$} \\
\hline 12 & Low & High & Nominal & Nominal & Nominal & \\
\hline 13 & High & Low & Nominal & Nominal & Nominal & \\
\hline 14 & High & High & \begin{tabular}{|l|} 
Nominal \\
\end{tabular} & \begin{tabular}{|l|} 
Nominal \\
\end{tabular} & Nominal & \\
\hline 15 & Low & Nominal & Low & Nominal & Nominal & \multirow{4}{*}{$\begin{array}{l}\text { Estimate interactive effect of } \\
\text { heat rate by Ne fraction }\end{array}$} \\
\hline 16 & Low & Nominal & High & Nominal & Nominal & \\
\hline 17 & High & Nominal & Low & Nominal & Nominal & \\
\hline 18 & High & Nominal & High & \begin{tabular}{|l|} 
Nominal \\
\end{tabular} & Nominal & \\
\hline 19 & Nominal & Low & Low & \begin{tabular}{|l|} 
Nominal \\
\end{tabular} & Nominal & \multirow{4}{*}{$\begin{array}{l}\text { Estimate interactive effect of } \\
\text { gas gap by } \mathrm{Ne} \text { fraction }\end{array}$} \\
\hline 20 & Nominal & Low & High & \begin{tabular}{|l|} 
Nominal \\
\end{tabular} & Nominal & \\
\hline 21 & \begin{tabular}{|l|} 
Nominal \\
\end{tabular} & High & Low & \begin{tabular}{|l|} 
Nominal \\
\end{tabular} & Nominal & \\
\hline 22 & \begin{tabular}{|l|} 
Nominal \\
\end{tabular} & High & High & \begin{tabular}{|l|} 
Nominal \\
\end{tabular} & Nominal & \\
\hline 23 & Low & Nominal & \begin{tabular}{|l|} 
Nominal \\
\end{tabular} & Low & Nominal & \multirow{4}{*}{$\begin{array}{l}\text { Estimate interactive effect of } \\
\text { heat rate by graphite } \\
\text { conductivity }\end{array}$} \\
\hline 24 & Low & Nominal & Nominal & High & Nominal & \\
\hline 25 & High & Nominal & Nominal & Low & Nominal & \\
\hline 26 & High & Nominal & \begin{tabular}{|l} 
Nominal \\
\end{tabular} & High & Nominal & \\
\hline 27 & \begin{tabular}{|l|} 
Nominal \\
\end{tabular} & Nominal & Low & Low & Nominal & \multirow{4}{*}{$\begin{array}{l}\text { Estimate interactive effect of } \\
\text { graphite conductivity by } \mathrm{Ne} \\
\text { fraction }\end{array}$} \\
\hline 28 & \begin{tabular}{|l|} 
Nominal \\
\end{tabular} & Nominal & High & Low & Nominal & \\
\hline 29 & \begin{tabular}{|l|} 
Nominal \\
\end{tabular} & Nominal & Low & High & Nominal & \\
\hline 30 & Nominal & Nominal & High & High & Nominal & \\
\hline 31 & Nominal & Low & Nominal & Low & Nominal & \multirow{4}{*}{$\begin{array}{l}\text { Estimate interactive effect of } \\
\text { gas gap by graphite conductivity }\end{array}$} \\
\hline 32 & Nominal & Low & Nominal & High & Nominal & \\
\hline 33 & Nominal & High & \begin{tabular}{|l} 
Nominal \\
\end{tabular} & Low & Nominal & \\
\hline 34 & \begin{tabular}{|l} 
Nominal \\
\end{tabular} & High & \begin{tabular}{|l} 
Nominal \\
\end{tabular} & High & Nominal & \\
\hline 35 & Low & Nominal & \begin{tabular}{|l} 
Nominal \\
\end{tabular} & \begin{tabular}{|l} 
Nominal \\
\end{tabular} & Low & \multirow{4}{*}{$\begin{array}{l}\text { Estimate interactive effect of } \\
\text { heat rate by fuel conductivity }\end{array}$} \\
\hline 36 & Low & Nominal & \begin{tabular}{|l} 
Nominal \\
\end{tabular} & Nominal & High & \\
\hline 37 & High & Nominal & Nominal & Nominal & Low & \\
\hline 38 & High & Nominal & Nominal & Nominal & High & \\
\hline 39 & Nominal & Low & Nominal & Nominal & Low & \multirow{4}{*}{$\begin{array}{l}\text { Estimate interactive effect of } \\
\text { heat rate by fuel conductivity }\end{array}$} \\
\hline 40 & \begin{tabular}{|l} 
Nominal \\
\end{tabular} & Low & \begin{tabular}{|l} 
Nominal \\
\end{tabular} & \begin{tabular}{|l} 
Nominal \\
\end{tabular} & High & \\
\hline 41 & \begin{tabular}{|l} 
Nominal \\
\end{tabular} & High & \begin{tabular}{|l} 
Nominal \\
\end{tabular} & \begin{tabular}{|l} 
Nominal \\
\end{tabular} & Low & \\
\hline 42 & Nominal & High & Nominal & Nominal & High & \\
\hline 43 & Nominal & Nominal & Low & Nominal & Low & \multirow{4}{*}{$\begin{array}{l}\text { Estimate interactive effect of } \\
\text { heat rate by fuel conductivity }\end{array}$} \\
\hline 44 & Nominal & Nominal & Low & Nominal & High & \\
\hline 45 & Nominal & Nominal & High & Nominal & Low & \\
\hline 46 & Nominal & Nominal & High & Nominal & High & \\
\hline 47 & Nominal & Nominal & Nominal & Low & Low & \multirow{4}{*}{$\begin{array}{l}\text { Estimate interactive effect of } \\
\text { heat rate by fuel conductivity }\end{array}$} \\
\hline 48 & Nominal & Nominal & Nominal & Low & High & \\
\hline 49 & Nominal & Nominal & Nominal & High & Low & \\
\hline 50 & Nominal & Nominal & Nominal & High & High & \\
\hline
\end{tabular}


Table 4. AGR-2 Capsule 5 thermal conditions for selected scenarios.

\begin{tabular}{|c|c|c|c|c|c|c|c|c|}
\hline & & $\begin{array}{c}\text { Control gap } \\
\text { distance } \\
\text { (in.) }\end{array}$ & $\begin{array}{c}\text { Fuel heat } \\
\text { rate } \\
\left(\mathrm{w} / \mathrm{cm}^{3}\right)\end{array}$ & $\begin{array}{c}\text { Fast } \\
\text { fluence } \\
\left(\mathrm{n} / \mathrm{m}^{2} 10^{25}\right)\end{array}$ & $\begin{array}{c}\text { Neon } \\
\text { fraction }\end{array}$ & $\begin{array}{c}\text { Volume } \\
\text { average } \\
\text { fuel } \\
\left({ }^{\circ} \mathrm{C}\right)\end{array}$ & $\begin{array}{c}\text { Peak } \\
\text { fuel } \\
\left({ }^{\circ} \mathrm{C}\right)\end{array}$ & $\begin{array}{c}\text { TC1 } \\
\left({ }^{\circ} \mathrm{C}\right)\end{array}$ \\
\hline 1 & $147 \mathrm{~A}-39.7$ & 0.01576 & 65.01 & 0.23391 & 0.690 & 1,082 & 1,207 & 952 \\
\hline 2 & $149 \mathrm{~A}-180.5$ & 0.01661 & 93.32 & 1.02364 & 0.477 & 1,153 & 1,281 & 963 \\
\hline 3 & $151 \mathrm{~A}-329.1$ & 0.01757 & 117.50 & 1.91886 & 0.274 & 1,207 & 1,350 & 980 \\
\hline 4 & $154 \mathrm{~B}-544.7$ & 0.01884 & 67.46 & 3.10006 & 0.936 & 1,212 & 1,304 & 1,046 \\
\hline 5 & $147 \mathrm{~A}-39.7$ & $\mathbf{0 . 0 3 1 3}$ & 65.01 & 0.23391 & 0.690 & 1,281 & 1,395 & 1,166 \\
\hline
\end{tabular}

\subsubsection{Thermal Model Parameter Sensitivity Analysis}

From the ABAQUS output for each run of the first four scenarios, multiple temperatures are obtained such as VA fuel temperature (FT), peak FT, and TC1 temperature in Capsule 5. These results are used for a sensitivity analysis of the input parameters on model predicted temperatures. The JMP ${ }^{\circledR}$ module $\left(\mathrm{JMP}^{\circledR}\right.$ 10.0.0) of $\mathrm{SAS}^{\circledR}\left(\mathrm{SAS}^{\circledR} 2009\right)$ is used to build a surrogate response surface model for each of the calculated temperatures in order to determine which input terms have significant impacts. The parameter coefficients are treated as sensitivities that estimate the rate of change of temperature with regard to the input. Subsequently, they can be used to propagate the parameter uncertainty to the output temperatures.

As stated in previous section, five thermal model input parameters are included in this sensitivity analysis, namely the fuel heat rate $(H R)$, the distance of the control gas gap $(G G)$ between the capsule graphite holder and the stainless steel test train wall, the neon fraction $(\mathrm{NeF})$ of the capsule gas mixture, graphite thermal conductivity $(G C)$, and fuel compact thermal conductivity $(F C)$. Since the experimental design provides estimation of main effects and pairwise interactions among input variables, the following response surface model containing 20 terms is constructed and studied for each of calculated temperature response measures using the JMP ${ }^{\circledR}$ platform:

$$
\begin{aligned}
f_{T}= & a_{0}+a_{1} H R+a_{2} G G+a_{3} N e f+a_{4} G C+a_{5} F C+a_{6} H R^{2}+a_{7} G G^{2}+a_{8} N e f^{2}+a_{9} G C^{2} \\
& +a_{10} F C^{2}+a_{11} H R \cdot G G+a_{12} H R \cdot N e f+a_{13} H R \cdot G C+a_{14} H R \cdot F C+a_{15} G G \cdot N e f \\
& +a_{16} G G \cdot G C+a_{17} G G \cdot F C+a_{18} N e f \cdot G C+a_{19} N e f \cdot F C+a_{20} G C \cdot F C
\end{aligned}
$$

Both input variables and output responses are transformed to a relative value ( 0.9 is $10 \%$ less, 1.0 is nominal, and 1.1 is $10 \%$ more) by dividing by the nominal values prior to response surface model fitting. This transformation does not impact the parameter sensitivities. Figure 19 presents the actual versus predicted plots for VA FT indicating near-perfect fit of the response surface regression model (R square nearly 1). 
Figure 20 lists all parameter estimates $\left(\mathrm{a}_{0}-\mathrm{a}_{20}\right.$ in Equation (10)) sorted from the largest to the smallest for VA fuel temperature in Scenario 1 (they are in the same order for other temperatures and other scenarios). Apparently, most of coefficients (parameter estimates) in the model are significantly different from zero, as indicated by the small Prob $>|t|$ values in the last column to the right. However, the bar chart showing the temperature variation due to input change indicates that only five main effects are dominant. The square of neon fraction (the most significant among the second order terms) has insignificant influence on output temperature. Similarly, Figure 21 shows the leverage plots for the five main effects (variables) and squared neon fraction for VA FT. The significant slopes of fuel heat rate, gas gap, and neon fraction are further indications of influence on VA FT variation. Although the estimate of the squared neon fraction term is significantly different from zero, its contribution to fuel temperature variation is small, as shown by the almost horizontal line in the leverage plot on bottom right. All other second-order terms in the regression model (Equation (10)) have even smaller contributions to the capsule temperature variations.

Additionally, the prediction profilers in the JMP "fit model" platform display profile traces for each independent variable and are shown in Figure 22 for VA fuel, peak fuel, and TC temperatures. A profile trace is the predicted response as one variable is changed while the others are held constant at their current values. The absence of curvature in prediction profiles for all variables confirms the linear relationship between response measures and input variables within 10\% of input variations.

The sensitivity analysis results presented in Figure 20, Figure 21, and Figure 22 allow for exclusion of all second order terms (square terms of five variables and their pairwise interactions) in the regression model (Equation (10)) because of their relatively negligible contributions to temperature variation. As a result, the regression model of Equation (10) can be reduced to a linearized approximation of model temperature as:

$$
f_{T}=a_{0}+a_{1} H R+a_{2} G G+a_{3} N e f+a_{4} G C+a_{5} F C
$$

The Equation (11) model's coefficient estimates $\left(\mathrm{a}_{0}-\mathrm{a}_{5}\right)$ for VA FT, peak FT, and temperatures at TC1 location for the five scenarios in Table 4 are presented in Table 5. Sensitivity of TC1 temperatures is also applied for TC2 temperature because they are located in a similar peripheral location of the graphite holder. The plots in Figure 23 show the variation of parameter sensitivities over different thermal conditions of the four selected scenarios throughout the irradiation period for Capsule 5. This reflects the nonlinear relationship between temperature and the thermal model inputs over the wide thermal condition range of the entire AGR-2 irradiation. Therefore, the model input sensitivities for other time steps will be estimated by interpolation from sensitivities of these four data sets depending on their actual thermal condition input parameters. The Capsule 5 input sensitivity coefficients will apply to corresponding calculated temperatures in other capsules. 
Table 5. Parameter estimates (sensitivity) for VA FT, peak FT and TC1 temperatures.

\begin{tabular}{|c|c|c|c|c|c|c|c|c|}
\hline Scenario & $\begin{array}{l}\text { Response } \\
\text { Variable }\end{array}$ & EFPD & $\begin{array}{c}\text { Intercept } \\
\left(\mathrm{a}_{0}\right)\end{array}$ & $\begin{array}{c}\text { Fuel Heat } \\
\text { Rate } \\
\left(a_{1}\right)\end{array}$ & $\begin{array}{c}\text { Gas Gap } \\
\left(\mathrm{a}_{2}\right)\end{array}$ & $\begin{array}{c}\text { Neon } \\
\text { Fraction } \\
\left(\mathrm{a}_{3}\right)\end{array}$ & $\begin{array}{c}\text { Graphite } \\
\text { Conduct } \\
\left(a_{4}\right)\end{array}$ & $\begin{array}{c}\text { Fuel } \\
\text { Conduct } \\
\left(a_{5}\right)\end{array}$ \\
\hline \multirow[t]{3}{*}{1} & VA FT & 39.7 & -0.015 & 0.457 & 0.299 & 0.356 & -0.058 & -0.039 \\
\hline & Peak FT & 39.7 & 0.122 & 0.483 & 0.248 & 0.311 & -0.080 & -0.083 \\
\hline & TC1 & 39.7 & -0.108 & 0.399 & 0.367 & 0.379 & -0.031 & -0.005 \\
\hline \multirow[t]{3}{*}{2} & VA FT & 180.5 & 0.112 & 0.533 & 0.272 & 0.206 & -0.069 & -0.053 \\
\hline & Peak FT & 180.5 & 0.223 & 0.542 & 0.237 & 0.181 & -0.086 & -0.095 \\
\hline & TC1 & 180.5 & -0.068 & 0.507 & 0.372 & 0.235 & -0.038 & -0.008 \\
\hline \multirow[t]{3}{*}{3} & VA FT & 329.1 & 0.229 & 0.556 & 0.252 & 0.102 & -0.075 & -0.064 \\
\hline & Peak FT & 329.1 & 0.343 & 0.545 & 0.218 & 0.087 & -0.088 & -0.104 \\
\hline & TC1 & 329.1 & 0.010 & 0.552 & 0.370 & 0.123 & -0.044 & -0.010 \\
\hline \multirow[t]{3}{*}{4} & VA FT & 544.7 & -0.013 & 0.469 & 0.200 & 0.433 & -0.051 & -0.036 \\
\hline & Peak FT & 544.7 & 0.092 & 0.462 & 0.185 & 0.387 & -0.062 & -0.063 \\
\hline & TC1 & 544.7 & -0.194 & 0.461 & 0.277 & 0.494 & -0.031 & -0.007 \\
\hline \multirow[t]{3}{*}{5} & VA FT & \multirow{3}{*}{\multicolumn{3}{|c|}{$\begin{array}{l}\text { Control gap sensitivity for } \\
\text { Capsule } 3 \text { control gap distance }\end{array}$}} & 0.192 & & & \\
\hline & Peak FT & & & & 0.172 & & & \\
\hline & TC1 & & & & 0.226 & & & \\
\hline
\end{tabular}

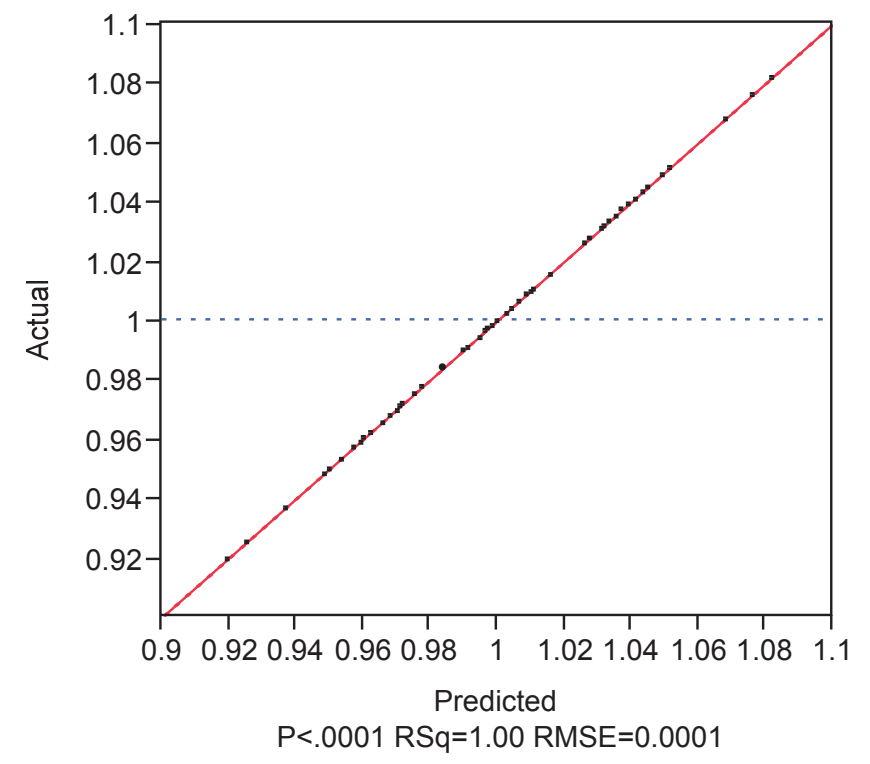

Figure 19. Actual by predicted plot for VA FT for Capsule 4. 


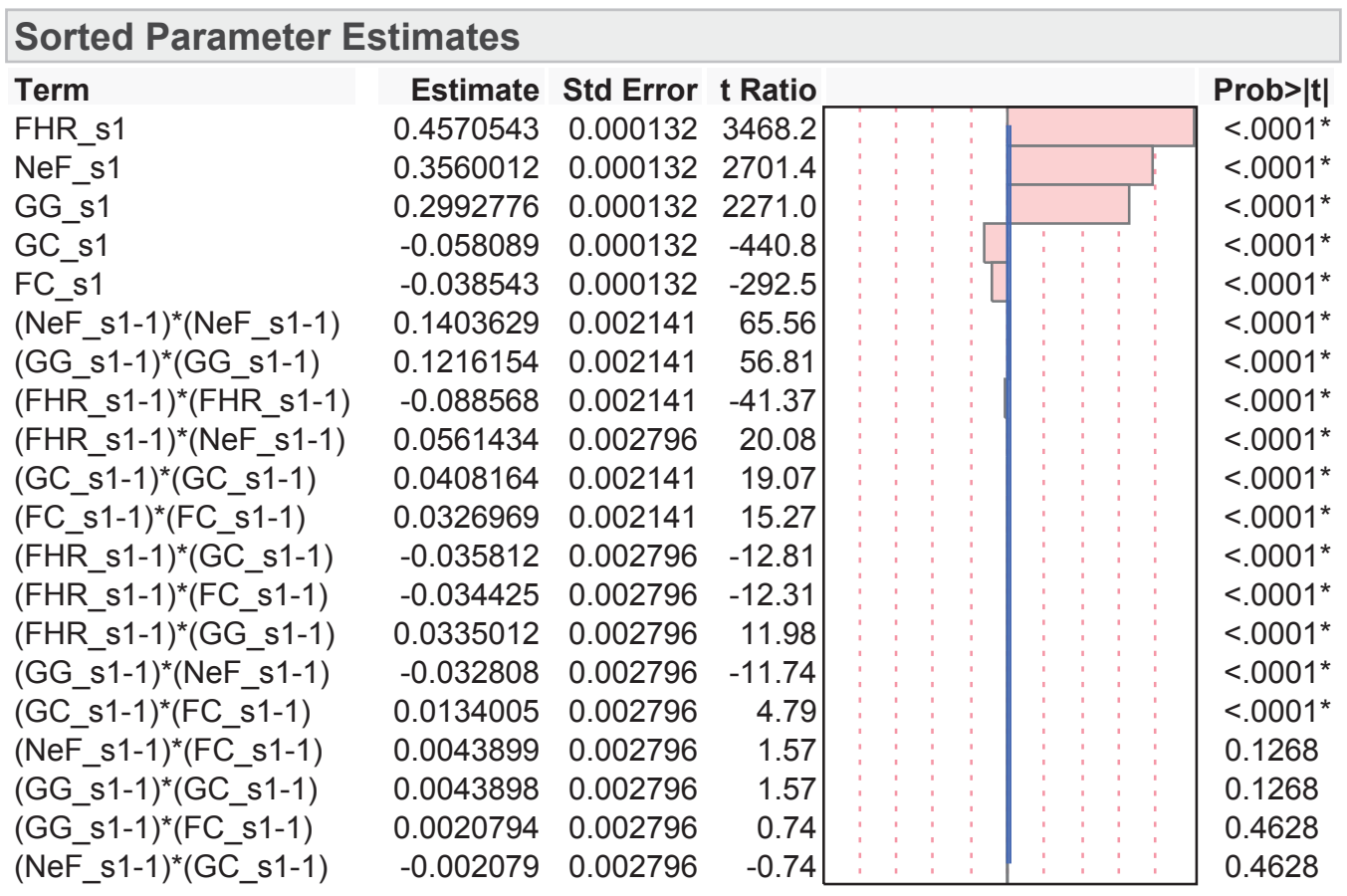

Figure 20. Parameter estimates (Equation (10)) sorted from the largest to the smallest for VA fuel temperature of Scenario 1.
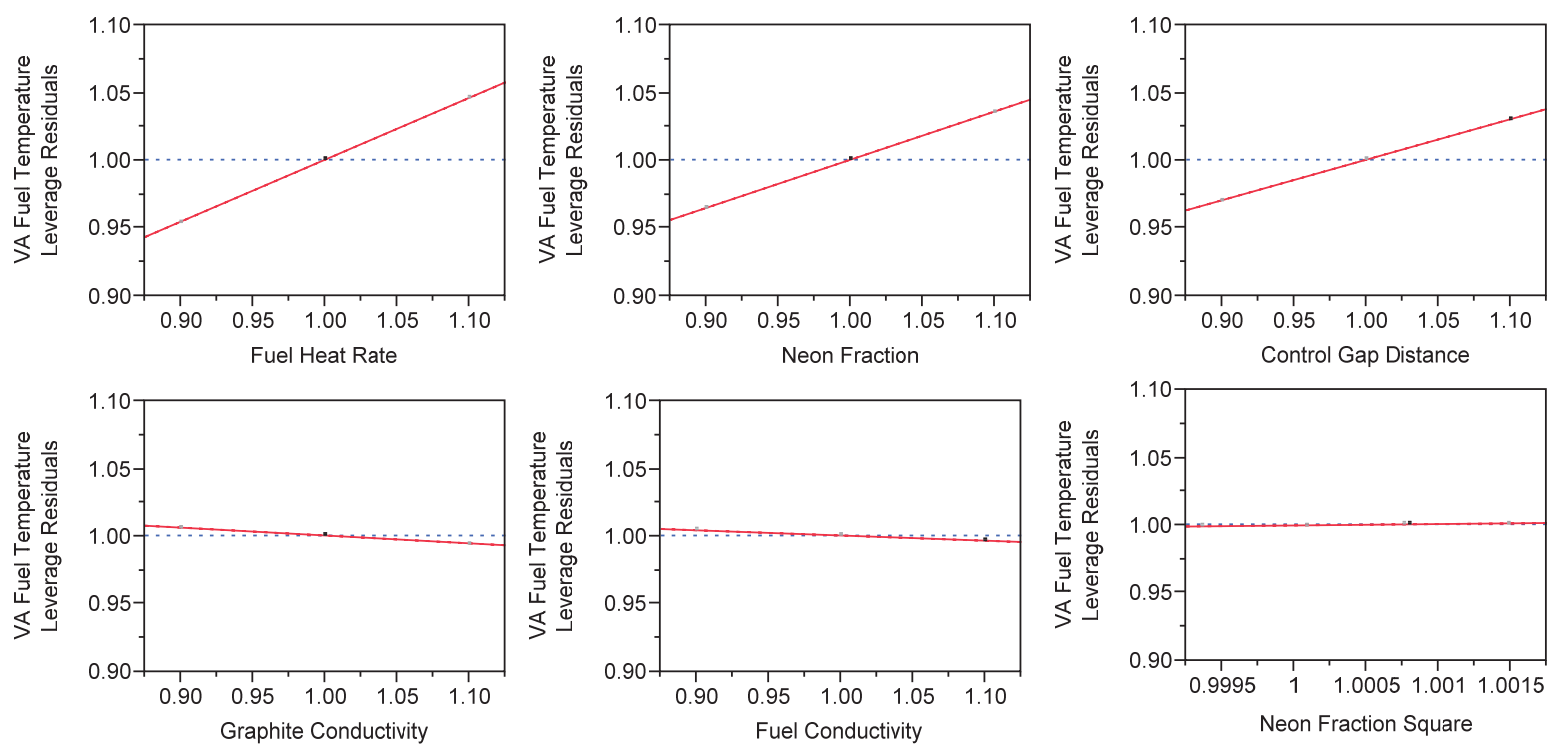

Figure 21. Leverage plots for five main effects and the square term of Ne fraction for VA FT (parameters are relative ratios to their nominal values). 


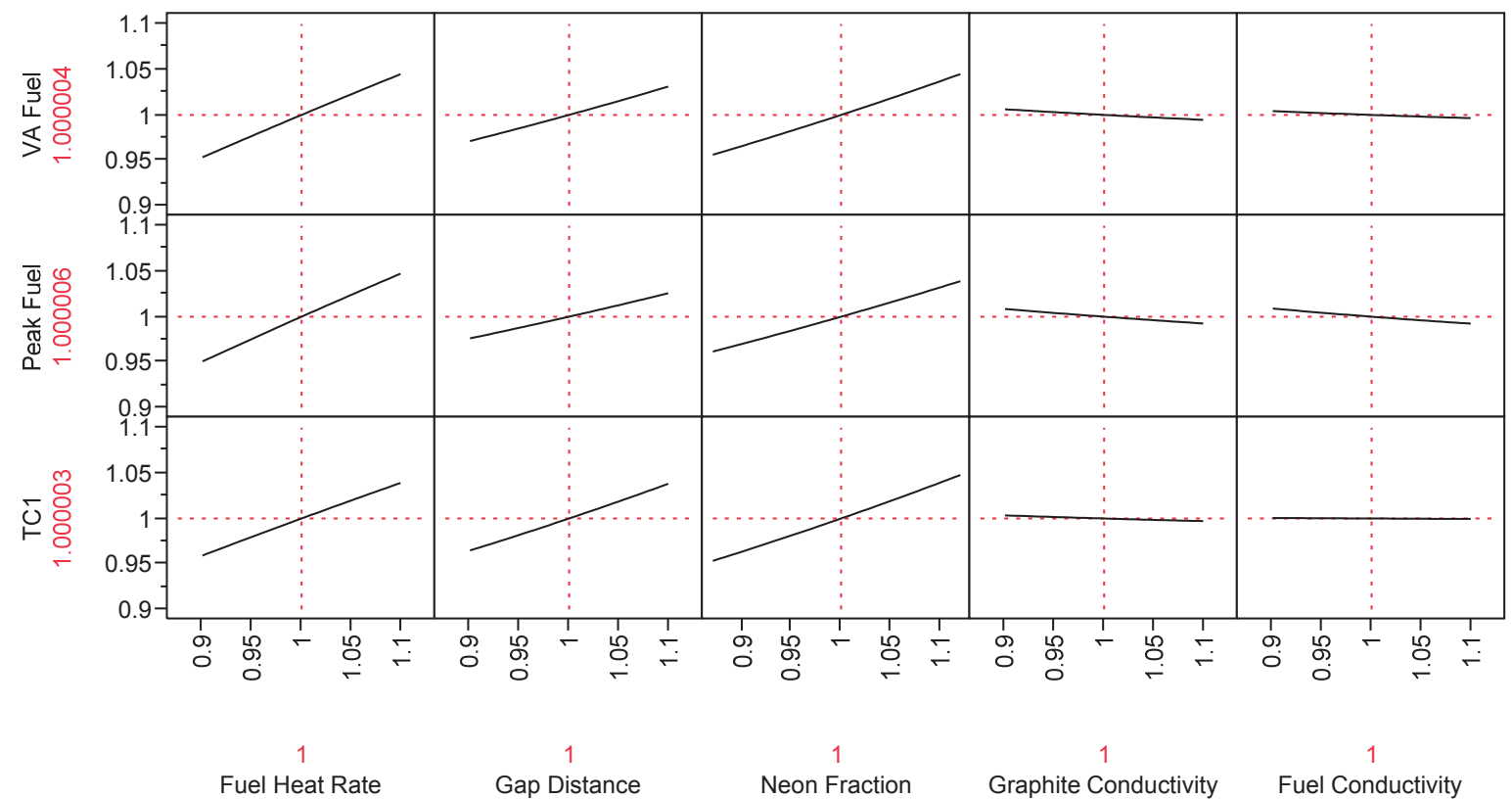

Figure 22. Prediction profiles of functions for VA fuel, peak fuel, and TC temperatures.

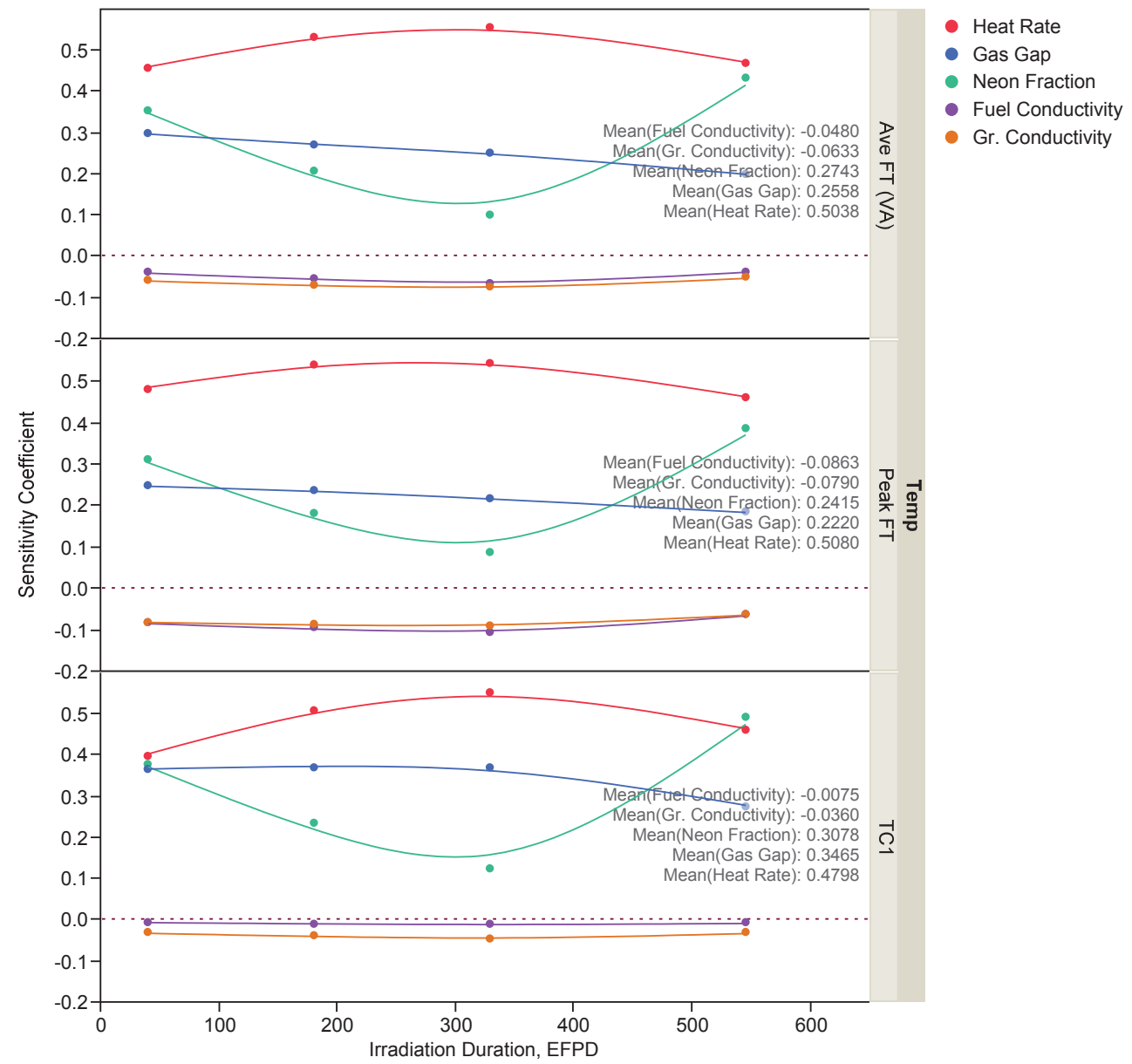

Figure 23. Sensitivity coefficients for fuel and TC temperatures as function of EFPD. 


\subsubsection{Interpolation of Sensitivity Coefficients for the Entire AGR-2 Irradiation}

In this section, the daily sensitivity coefficients for the entire AGR-2 irradiation are estimated from four sensitivity data points in Table 5 (five for control gap distance) by interpolation. For instance, Table 6 presents the fuel heat rate sensitivity coefficients for VA FT, peak FT, and TC1 temperature for the four the fuel heat rate values in Capsule 5 corresponding to four selected scenarios.

Table 6. Sensitivity coefficients of fuel heat rate for thermocouples, volume average fuel, and peak fuel temperature.

\begin{tabular}{|c|c|c|c|c|}
\hline \multirow{2}{*}{ Scenario } & \multirow{2}{*}{$\begin{array}{c}\text { Heat Rate } \\
\left(\mathrm{w} / \mathrm{cm}^{3}\right)\end{array}$} & VA FT & Peak FT & TC1 \\
\cline { 3 - 5 } & 65.01 & 0.457 & 0.483 & 0.399 \\
\hline 1 & 93.32 & 0.533 & 0.542 & 0.507 \\
\hline 2 & 117.5 & 0.556 & 0.545 & 0.552 \\
\hline 3 & 67.46 & 0.469 & 0.462 & 0.461 \\
\hline 4 & & & & \\
\hline
\end{tabular}

The fitting procedure of the EXCEL (Excel 2007 SP3) trend line feature is used to construct the polynomial function of input parameter $(\mathrm{x})$ for each input sensitivity $(\mathrm{y})$. The functional form was selected as follows:

(1) The fuel heat rate sensitivities are fit well using a second order polynomial function of fuel heat rate (as shown in Figure 24) over the wide range of fuel heat rate for all temperatures.

(2) The neon fraction sensitivities are also fit well with a second order polynomial function of neon fraction with restricted zero intercept (Figure 25). The zero intercept restriction prevents neon fraction sensitivity becoming negative when neon fraction is close to zero.

(3) The control gas gap sensitivities are acceptably fit with a second order polynomial function of the gap distance (Figure 26). The additional data point for the biggest control gas gap in Capsule 3 (points on the far right at $0.31 \mathrm{in}$.) allows interpolation of the gas gap sensitivity for other time steps and capsules.

(4) The graphite conductivity sensitivities (Figure 27) and the fuel compact conductivity sensitivities (Figure 28) are fit very well by a second order polynomial function of accumulative fast fluence over the whole range fast fluence in all capsules. 


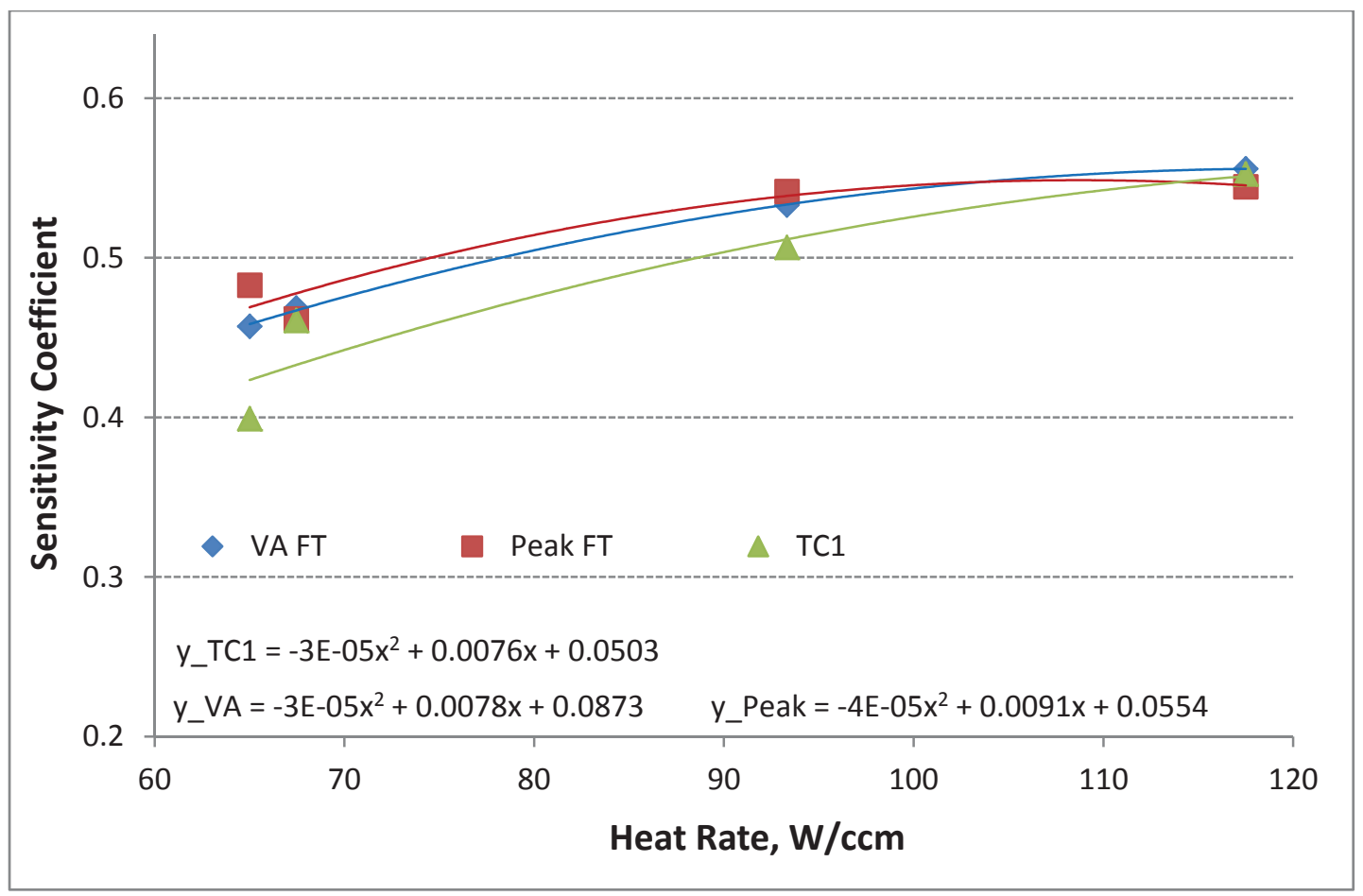

Figure 24. Heat rate sensitivity for thermocouples and fuel temperatures as function of heat rate.

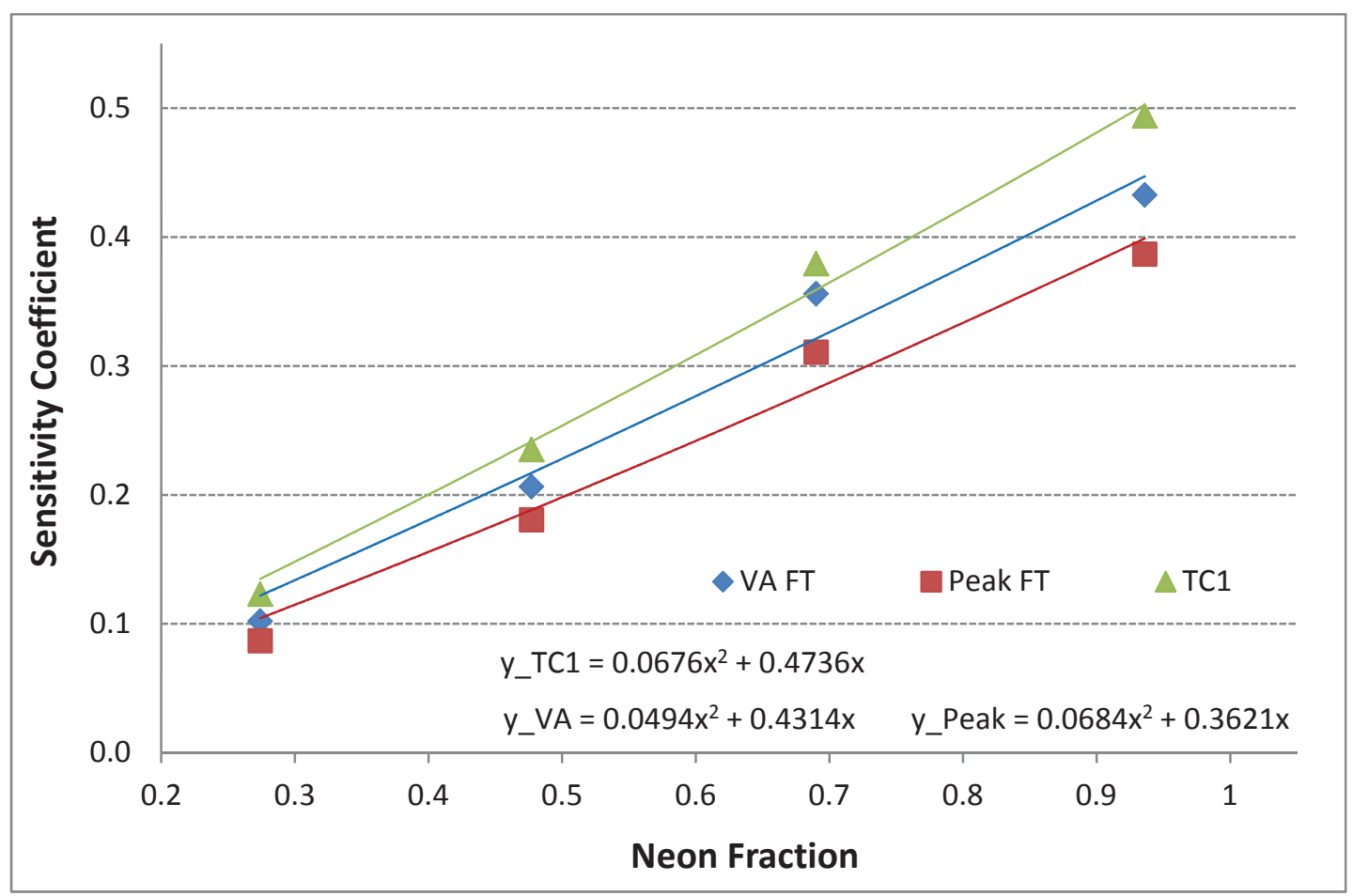

Figure 25. Neon fraction sensitivity for thermocouples and fuel temperatures as function of neon fraction. 


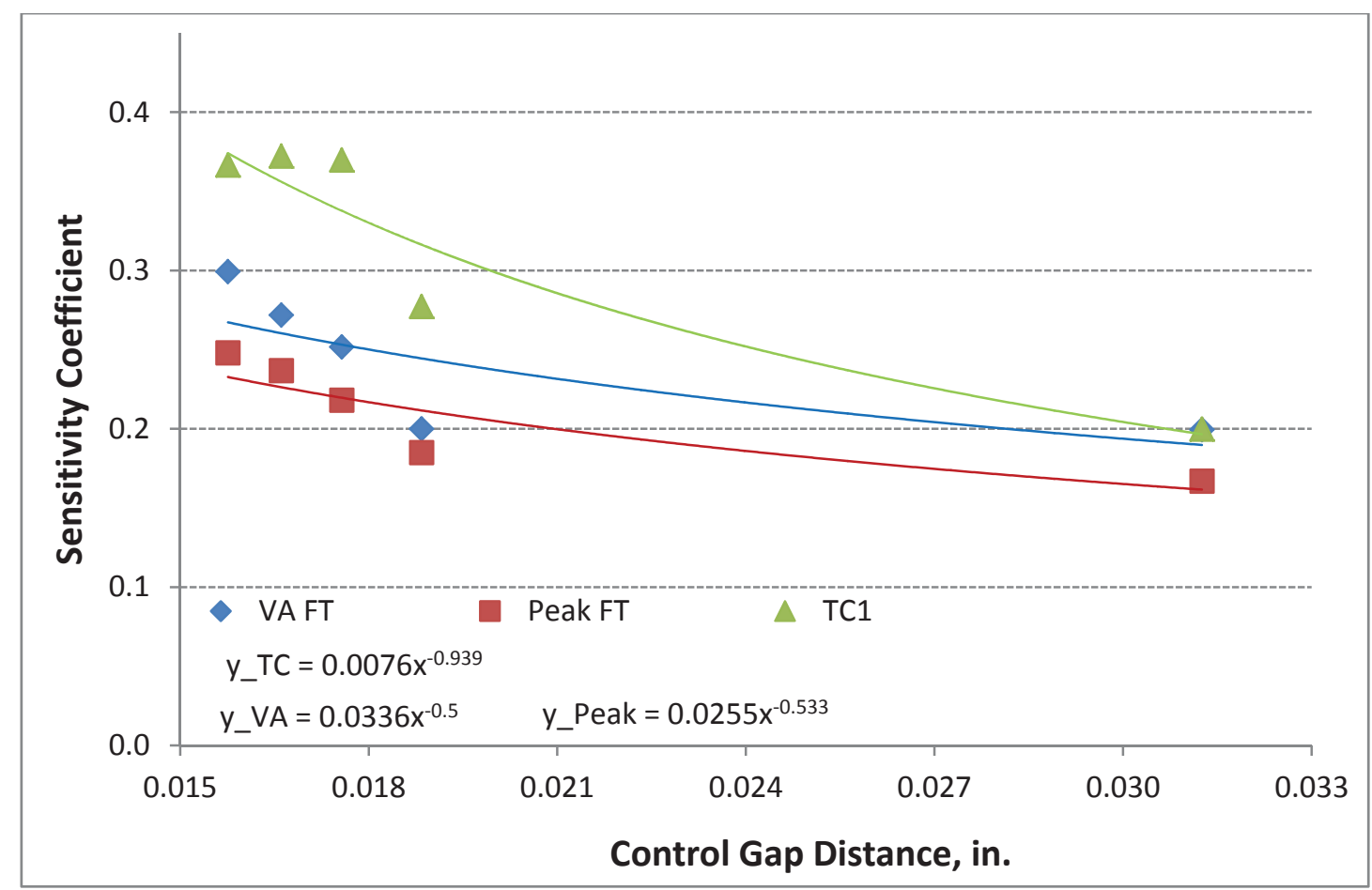

Figure 26. Gas gap sensitivity for thermocouples and fuel temperatures as function of gap distance.

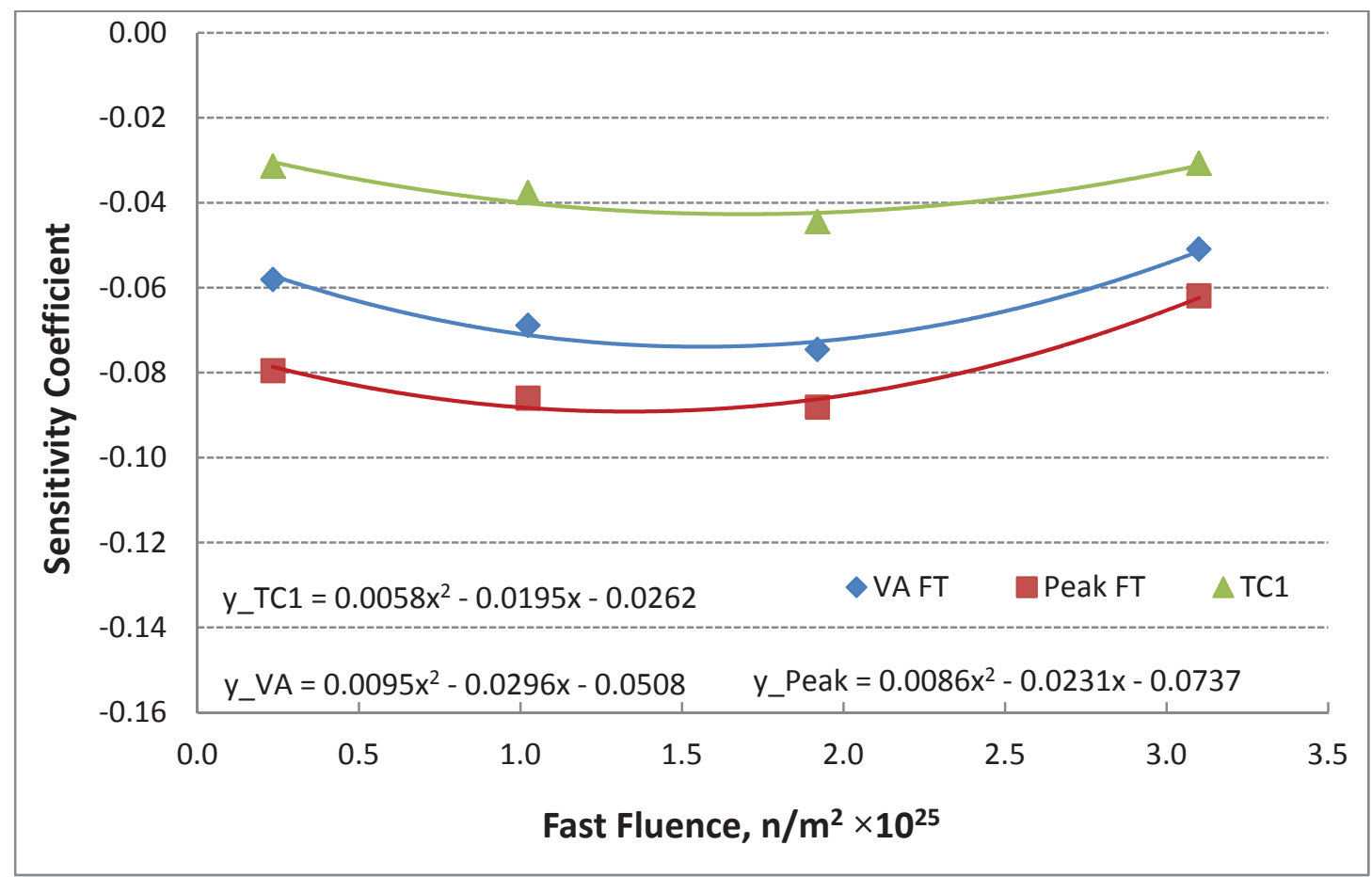

Figure 27. Graphite conductivity sensitivity for thermocouples and fuel temperatures as function of fluence. 


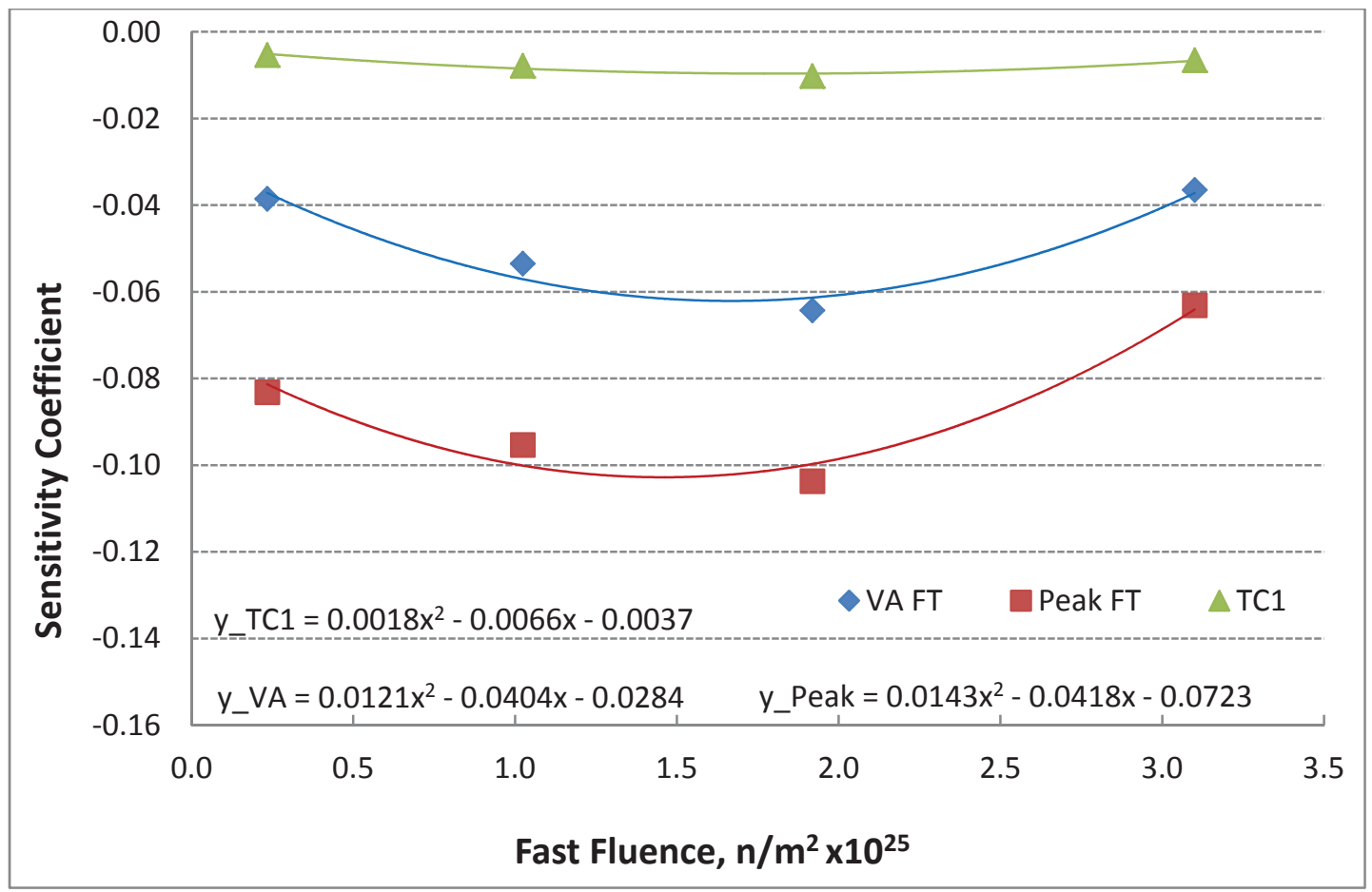

Figure 28. Fuel conductivity sensitivity for thermocouples and fuel temperatures as function of fluence.

\subsection{Correlation Coefficients of Thermal Model Input Parameters}

\subsubsection{Uncorrelated Parameter Errors}

The correlation coefficient between two input parameters equals zero when their error sources are independent or uncorrelated. In other words, the variation of one parameter due to its random error does not affect the error of other parameter. For example, initial gas gap distance and neon fraction are both the result of measurements performed by different tools and procedures. The flow rate is measured by the flow meter and the control gas gap is based on measuring physical dimensions of capsules compacts. Therefore, the error of the gas gap measurement does not affect the error in flow rate measurement and these errors are considered independent. As a result, the correlation coefficient of neon fraction and gas gap distance is zero. For the same reason, correlation coefficients between the two measured parameters, gas gap distance and neon fraction, and three calculated parameters, fuel heat rate, graphite, and fuel compact thermal conductivities, are zero.

\subsubsection{Correlated Parameter Errors}

This section estimates the correlation coefficients between error sources of three calculated parameters: fuel heat rate, graphite conductivity, and fuel compact conductivity. The error associations of these calculated parameters are caused by the fact that their calculation formulas contain common factors such as temperature and fast neutron fluence. 


\subsubsection{Graphite and Fuel Compact Thermal Conductivities}

The graphite thermal conductivity and fuel compact thermal conductivity plots varying with temperature and fast fluence (expressed in $\mathrm{n} / \mathrm{cm}^{2}$ or equivalent $\mathrm{dpa}$ ) are presented in Figure 17 and Figure 18, respectively. From available data provided by the look-up tables for fuel and graphite conductivities as functions of fast fluence and temperature (Hawkes et al. 2011), the relationship of graphite and fuel compact thermal conductivities as functions of fast fluence (converted into $d p a$ ) and temperature $(T)$ are constructed using JMP ${ }^{\circledR}$. The functional relationship given for graphite thermal conductivity is:

$$
\begin{aligned}
G C= & 3.36 \cdot 10^{-4}-3.85 \cdot 10^{-8} T+1.02 \cdot 10^{-8}(T-1449.5)(\log (d p a)+5.05) \\
& -4.499 \cdot 10^{-5} \log (d p a)-2.36 \cdot 10^{-6}(\log (d p a)+5.05)^{2}
\end{aligned}
$$

and for fuel compact conductivity is:

$$
\begin{aligned}
F C= & 3.72 \cdot 10^{-5}+4.07 \cdot 10^{-8} T-9.44 \cdot 10^{-6} d p a+3.74 \cdot 10^{-11}(T-2030)^{2} \\
& +2.31 \cdot 10^{-8}(T-2030)(d p a-1.82)+2.85 \cdot 10^{-6}(d p a-1.82)^{2}
\end{aligned}
$$

Using JMP ${ }^{2}$, a total of 100,000 data points was collected from normal distributions of AGR-2 FT $(1500 \pm 200 \mathrm{~K})$, graphite temperature $(1200 \pm 200 \mathrm{~K})$, and $d p a\left(\mu_{\mathrm{dpa}} \pm 10 \%\right)$ for five levels of the mean $d p a, \mu_{\mathrm{dpa}}=0.5,1,2,3,4$ corresponding to fast neuron fluence. The multiplier used to convert fast fluence to $d p a$ is $0.823 \times 10^{25} \mathrm{dpa} /\left(\mathrm{n} / \mathrm{m}^{2}\right)$ (Hawkes et al. 2011).

To compute the noise correlation coefficient between fuel and graphite conductivities, the fuel and graphite temperatures of one data point (a pair of temperature and $d p a$ ) are sampled with the same noise components. The sampled dpa and temperature values are inserted in (Equation (12)) and (Equation (13)) to compute the graphite thermal conductivity and the fuel compact thermal conductivity for each data point. The correlation coefficient between graphite and fuel compact thermal conductivities is estimated using the JMP ${ }^{\circledR}$ "multivariate" function. Figure 29 shows the scatter plot matrix of FT, $d p a$, fuel and graphite thermal conductivities together with their distribution and correlation coefficients of each pair of variables for $d p a$ levels 0.5 and 2.0. 

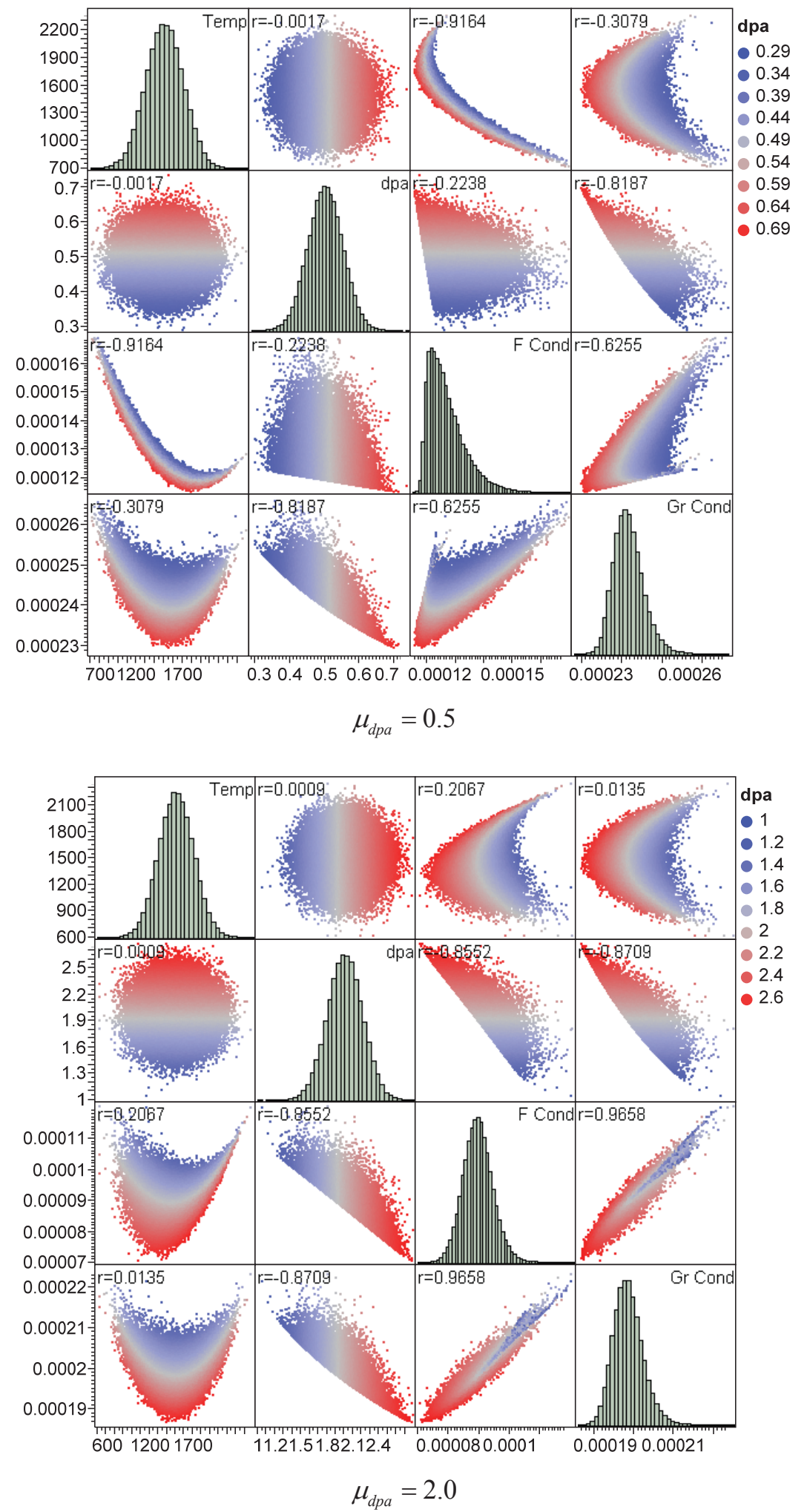

Figure 29. Scatter plot matrix shows the correlation between fuel and graphite thermal conductivities at one fuel temperature and two $d p a$ levels (0.5 and 2.0). 
The noise correlation coefficients between fuel and graphite thermal conductivities $\rho_{G C, F C}$ for five levels of fast neutron fluence (converted from $d p a$ ) are presented in Table 7 and Figure 30. The second order polynomial function of the fast fluence $(f)$ in $\mathrm{n} / \mathrm{m}^{2} \times 10^{25}$ was used to fit the correlation coefficients in Table 7 as:

$$
\rho_{G C, F C}=-0.0708 f^{2}+0.3585 f+0.4464
$$

Table 7. Correlation coefficients between graphite and fuel thermal conductivities.

\begin{tabular}{|l|c|c|c|c|c|}
\hline fluence $\left(\mathrm{n} / \mathrm{m}^{2} \times 10^{25}\right)$ & 0.6075 & 1.215 & 2.430 & 3.645 & 4.860 \\
\hline Correlation coefficient & 0.6255 & 0.7739 & 0.9658 & 0.7355 & 0.544 \\
\hline
\end{tabular}

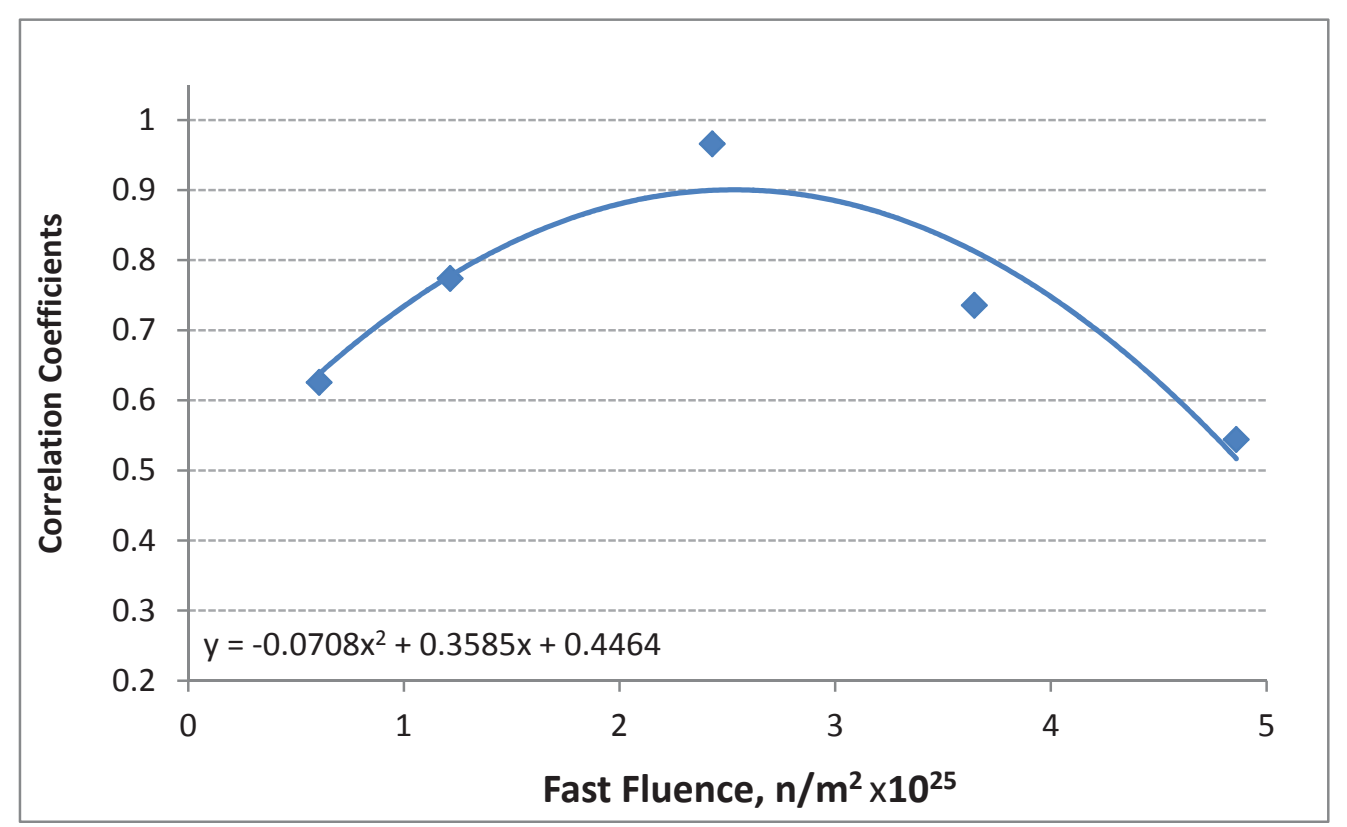

Figure 30. Correlation coefficients between graphite and fuel thermal conductivities varying with fast neutron fluence.

\subsubsection{Graphite/Fuel Compact Thermal Conductivity and Fuel Compact Heat Rate}

The value of fuel compact heat rate $(Q)$ depends on the daily neutron flux, FT, and material properties such as cross-section and fuel burnup. The graphite and fuel compact thermal conductivity, as mentioned earlier, is a function of temperature and neutron fast fluence. Based on the functional dependence of fuel compact and graphite thermal conductivity and fuel heat rate on neutron fast fluence and neutron flux respectively, it is reasonable to assume that the correlation coefficient between their noises should be smaller than between graphite and fuel conductivities. Since the fuel compact and graphite thermal conductivities and fuel heat rate are not possible to simulate, their noise correlation coefficients will assume to be half of the correlation coefficient between fuel and graphite conductivities. 


\section{PROPAGATION OF PARAMETER UNCERTAINTIES AND SENSITIVITIES}

\subsection{Propagation Formula}

The variation of parameter estimates across three thermal condition scenarios in Capsule 5 presented in Figure 23 reflects the variation in parameter sensitivities over the entire input domain for AGR-2. This confirms the nonlinear nature of Capsule 5 thermal models as a function of the five input variables. However, within a small enough input range (e.g., from [nominal* $(1-10 \%)]$ to $\left[\right.$ nominal $\left.^{*}(1+10 \%)\right]$ ) the AGR-2 predicted temperature can be estimated by a linear combination of five input variables as presented in Equation (11). Subsequently, the standard parameter error propagation of the linear summation (Ostle and Mensing 1975) can be used to calculate the overall output temperature uncertainty from the variance-covariance matrix of input variables for an input range close to the nominal values. The expression of output variance for each output, $T$, is given in Section 3.1 and reproduced here:

$$
\sigma_{T}^{2}=\sum_{i}^{n} a_{i}^{2} \sigma_{i}^{2}+\sum_{i}^{n} \sum_{j \neq i}^{n} \rho_{i j} a_{i}^{2} \sigma_{i}^{2} a_{j}^{2} \sigma_{j}^{2}
$$

where

$\sigma_{T}$ is the overall uncertainty in terms of standard deviation

$a_{i}$ is the sensitivity coefficient for parameter $i$

$\sigma_{i}$ is the uncertainty of input parameter $i$ in terms of standard deviation

$\rho_{i j}$ is the correlation coefficient for input parameters $i$ and $j$.

The input parameter uncertainties are estimated in terms of relative standard deviations (\%) in Section 3.3 and the sensitivity coefficients are estimated in Section 3.4. Section 3.5 addresses the correlation coefficients of each variable pair. The correlation coefficient of a variable pair is a measure of the degree (or intensity) of association between two variables (Ostle and Mensing 1975).

\subsection{Uncertainty Quantification for Daily Average Temperatures}

For each capsule, the AGR-2 thermal model parameter uncertainty quantification for daily average temperatures (TCs, VA FT, or peak FT) is performed using the results presented in Section 2.

\subsubsection{Daily Input Parameter Uncertainties $\left(\sigma_{i}\right)$}

The daily input parameter uncertainties in terms of relative standard deviation for the five significant input variables are estimated as discussed in Section 3.3. The details of the daily uncertainty calculations are:

- The parameter uncertainties for fuel heat rate, graphite holder thermal conductivity and fuel compact thermal conductivity are assumed to be constant random errors for all time steps and six capsules as presented in Table 1. Therefore, their plots as a function of EFPDs are flat lines for all three parameters in all capsules.

- The daily parameter uncertainties for gas gap distance $\left(\sigma_{G G i}\right)$ are based on the error of the gap distance formula of Equation (6).

- The daily parameter uncertainty for neon fraction $\left(\sigma_{\text {Nef }_{i}}\right)$ is calculated for each time step and each capsule by substituting the actual capsule neon fraction on that time step using Equation (8) (representing a functional relationship between neon fraction uncertainties and neon fraction values). Since the capsule neon fractions vary with time in order to maintain the capsule specified FT, the daily uncertainties of neon fraction also vary with EFPDs. 


\subsubsection{Daily Parameter Sensitivities $\left(a_{i}\right)$}

The daily parameter sensitivities, $a_{i}$, of five significant input variables at time step (i) are estimated using the functional relationships between sensitivity coefficients and corresponding input parameters established in Section 3.4 for each output temperature in each capsule. Since the inputs to the thermal models vary with time, the resulting daily parameter sensitivities also vary with time.

\subsubsection{Daily Correlation Coefficients $\left(\sigma_{F C, G C_{i}}\right)$}

The daily correlation coefficients between fuel compact and graphite thermal conductivities, $\rho_{F C, G C}$, at time step (i), are calculated using the function of fast neutron fluence given in Equation (14) and shown in Figure 30. Since the neutron fluence varies with time, the daily correlation coefficients also vary with time.

\subsubsection{Overall Parameter Uncertainty $\left(\sigma_{T_{i}}^{2}\right)$}

The overall uncertainty (in terms of variance) of the thermal model output temperature at time step (i) is calculated using Equation (15), which is based on propagation of Equation (11):

$$
\begin{gathered}
\sigma_{T_{i}}^{2}=\left(a_{H R_{i}} \sigma_{H R_{i}}\right)^{2}+\left(a_{G G_{i}} \sigma_{G G_{i}}\right)^{2}+\left(a_{N e f_{i}} \sigma_{N e f_{i}}\right)^{2}+\left(a_{G C_{i}} \sigma_{G C_{i}}\right)^{2}+\left(a_{F C_{i}} \sigma_{F C_{i}}\right)^{2}+2 \rho_{F C, G C_{i}} a_{G C_{i}} a_{F C_{i}} \sigma_{G C_{i}} \sigma_{F C_{i}} \\
+2 \rho_{H R_{i}, G C_{i}} a_{G C_{i}} a_{H R_{i}} \sigma_{G C_{i}} \sigma_{H R_{i}}+2 \rho_{F C_{i}, H R_{i}} a_{F C_{i}} a_{H R_{i}} \sigma_{H R_{i}} \sigma_{F C_{i}}
\end{gathered}
$$

Since the input sensitivity, correlation coefficient and uncertainty vary with time, the calculated temperature uncertainty also varies with time (EFPDs).

\subsection{Uncertainty Quantification for Time Average (TA) Temperatures}

The capsule TA VA FT and TA peak FT at day $(i)$ are calculated from daily average temperatures as

$$
T_{T A_{i}}=\frac{\sum_{k=1}^{i} T_{k} \cdot t_{k}}{\sum_{k=1}^{i} t_{k}}
$$

where $T_{k}$ and $t_{k}$ are daily average calculated temperature and time step at day $(k)$. Time step $t_{k}$ equals 1 for most time steps and $t_{k}<1$ for the startup or power-down days. The error standard deviation of the TA temperatures is calculated using the standard formula (Equation (17)) for error propagation of the linear combination of daily average temperatures. Assuming that the errors of daily averaged calculated temperatures are independent from each other, the error standard deviation of the TA FT (VA or peak) is calculated as

$$
\sigma_{T_{T A i}}=\sqrt{\frac{\sum_{k=1}^{i}\left(t_{k} \sigma_{T_{k}}\right)^{2}}{\sum_{k=1}^{i} t_{k}}}
$$




\section{RESULTS}

The overall model temperature uncertainty is estimated using the uncertainty quantification procedure described in the previous sections. The daily uncertainty (in terms of relative and absolute standard deviation at each time step) is estimated for following calculated temperatures: peripheral TCs, instantaneous VA FT, peak FT, time-average VA FT, and time-average peak FT.

As stated in Section 3.3, the uncertainties of fuel heat rate and fuel and graphite thermal conductivities are assumed constant over the entire irradiation and are the same for all six AGR-2 capsules. Therefore they are plotted as horizontal lines as a function of EFPDs. The fuel conductivity has highest uncertainty followed by the graphite conductivities, while uncertainties for fuel heat rate, control gas gap, and neon fraction are similar and approximately 5\%. The uncertainties of neon fraction and control gas gap distance are dynamic over irradiation time because they are a function of the input variations as described in the Subsections 3.3.1 and 3.3.2. It is also observable that the neon fraction uncertainties are unusually large (they are out of the plotting limits as seen by the green triangles in the input uncertainty plots) for a few time steps at the beginning and the end of each ATR cycle when the neon fractions were close to zero.

The dominant parameter of the predicted temperature uncertainty is the parameter that contributes to the largest temperature variance. In turn, the temperature variance due to input uncertainty is the square of the product of the sensitivity coefficient and the standard deviation for a parameter $\left(a_{i} \sigma_{i}\right)^{2}$. The dominant parameter varies depending on input parameter uncertainty (e.g., fuel heat rate, neon fraction) and output temperatures (TC, VA FT, and peak FT). The results given in the following sections identify the dominant parameter and quantify its influence on the overall uncertainty. The dominant sensitivity is the range of the sensitivity coefficients for the driver input over all EFPDs. The dominant weighted variance is the range of the relative temperature variances due to the dominant input uncertainty. The overall uncertainties in terms of standard deviation are given both as a range over EFPDs for instantaneous temperatures and at the end of irradiation for time-average temperatures.

For all capsules, the overall temperature uncertainties are higher around the middle of irradiation because the highest sensitivity of fuel heat rate (dominant factor) occurred when the fuel heat rate was at the peak level. At the same time, the doubled neon fraction uncertainty due to the gas line cross-talk failure during ATR Cycle 150B, which has the same impact on temperature uncertainty as the fuel heat rate, leads to the highest level of temperature uncertainty. Conversely, the notable drop in temperature uncertainty around 350 EFPD (ATR Cycle 151A) is caused by low neon fraction uncertainty when the AGR-2 experiment was run on pure helium. The drop in temperature uncertainty during PALM Cycle 153B (around 450 EFPD) is caused by low heat rate sensitivity at low fuel heat rate as the AGR-2 experiment was moved to peripheral location I-24. The last drop in temperature uncertainty during the first half of ATR Cycle 154B is due to near zero neon fraction uncertainty when the AGR-2 test was run on pure neon, along with lower fuel heat rate sensitivity at low heat rate at the end of irradiation.

\subsection{Temperature Uncertainty Results for Capsule 6}

Table 8 summarizes the results for the top capsule, Capsule 6 . The following results of calculated temperature uncertainty are observed:

- Input parameter uncertainty: The daily input standard deviations for five significant input parameters for Capsule 6 are presented in Figure 31. The neon fraction uncertainties (green triangles) are in the range between $0 \%$ and $17 \%$ and the control gas gap distance uncertainty (blue line) is gradually increasing from the smallest uncertainty of $4.3 \%$ for initial gap distance to $6.5 \%$ at the end of irradiation. This is because Capsule 6 has relatively large control gap distance leading to smaller gap uncertainty.

- Input parameter sensitivity: The daily input sensitivities for VA FT, peak FT, and TC temperatures as a function of EFPDs are plotted in Figure 32. As expected, the fuel heat rate sensitivities (red dots) 
are the greatest for most days of irradiation and sensitivities of fuel and graphite thermal conductivity (orange lines) are smallest, especially for temperatures at the peripheral TC location (top plot).

- Weighted variance (Figure 33): The dominant factor for model temperature uncertainty is the $5 \%$ error in the fuel heat rate (highest sensitivity coefficient and relatively high input uncertainty) for all calculated temperatures. This contributed up to $8.1 \%$ of uncertainty in terms of variance for peak FT.

- Overall calculated temperature uncertainty: The daily relative and absolute standard deviations of TC and fuel compact temperature model uncertainty are presented in Figure 34 and Figure 35. The lower uncertainty standard deviations at the beginning and the end of each ATR cycle are due to lower input values leading to lower parameter sensitivities for these time steps. The overall uncertainties of peak FT (light-brown triangles) are the highest up to $3.9 \%$ (or $50^{\circ} \mathrm{C}$ ).

The daily temperature and one standard deviation of the predicted temperatures in Capsule 6 are plotted for peripheral TCs in Figure 36, daily average VA FT and daily peak FT in Figure 37, and TA VA FT and TA peak FT in Figure 38.

Table 8. Summary of temperature uncertainty results for Capsule 6 .

\begin{tabular}{|l|c|c|c|c|c|}
\hline & TC & VA FT & Peak FT & $\begin{array}{c}\text { TA VA FT } \\
\text { at EOE }\end{array}$ & $\begin{array}{c}\text { TA Peak } \\
\text { FT at EOE }\end{array}$ \\
\hline \multicolumn{1}{|c|}{ Dominant parameter } & $\begin{array}{c}\text { Fuel } \\
\text { Heat Rate }\end{array}$ & $\begin{array}{c}\text { Fuel } \\
\text { Heat Rate }\end{array}$ & $\begin{array}{c}\text { Fuel } \\
\text { Heat Rate }\end{array}$ & & \\
\hline Dominant sensitivity & $0.3-0.52$ & $0.37-0.58$ & $0.35-0.57$ & & \\
\hline $\begin{array}{l}\text { Dominant weighted } \\
\text { variance, } \%\end{array}$ & $2.6-6.7$ & $3.0-8.3$ & $3.0-8.1$ & & \\
\hline Relative uncertainty, \% & $2.3-3.6$ & $2.6-3.6$ & $2.8-3.9$ & 2.9 & 3.4 \\
\hline Absolute uncertainty, ${ }^{\circ} \mathrm{C}$ & $18-38$ & $24-42$ & $31-50$ & 32 & 40 \\
\hline
\end{tabular}

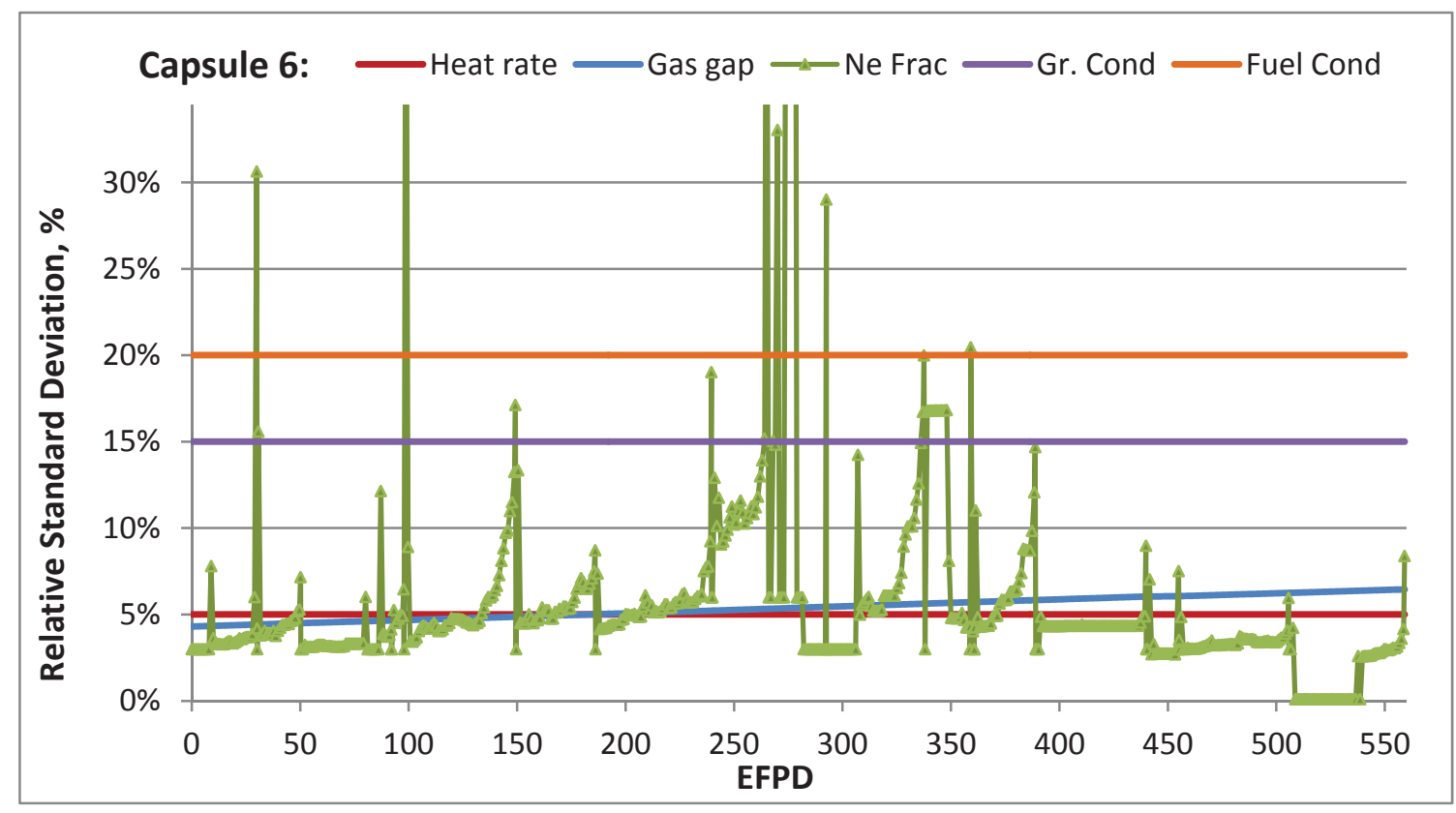

Figure 31. Daily input relative standard deviations for Capsule 6. 

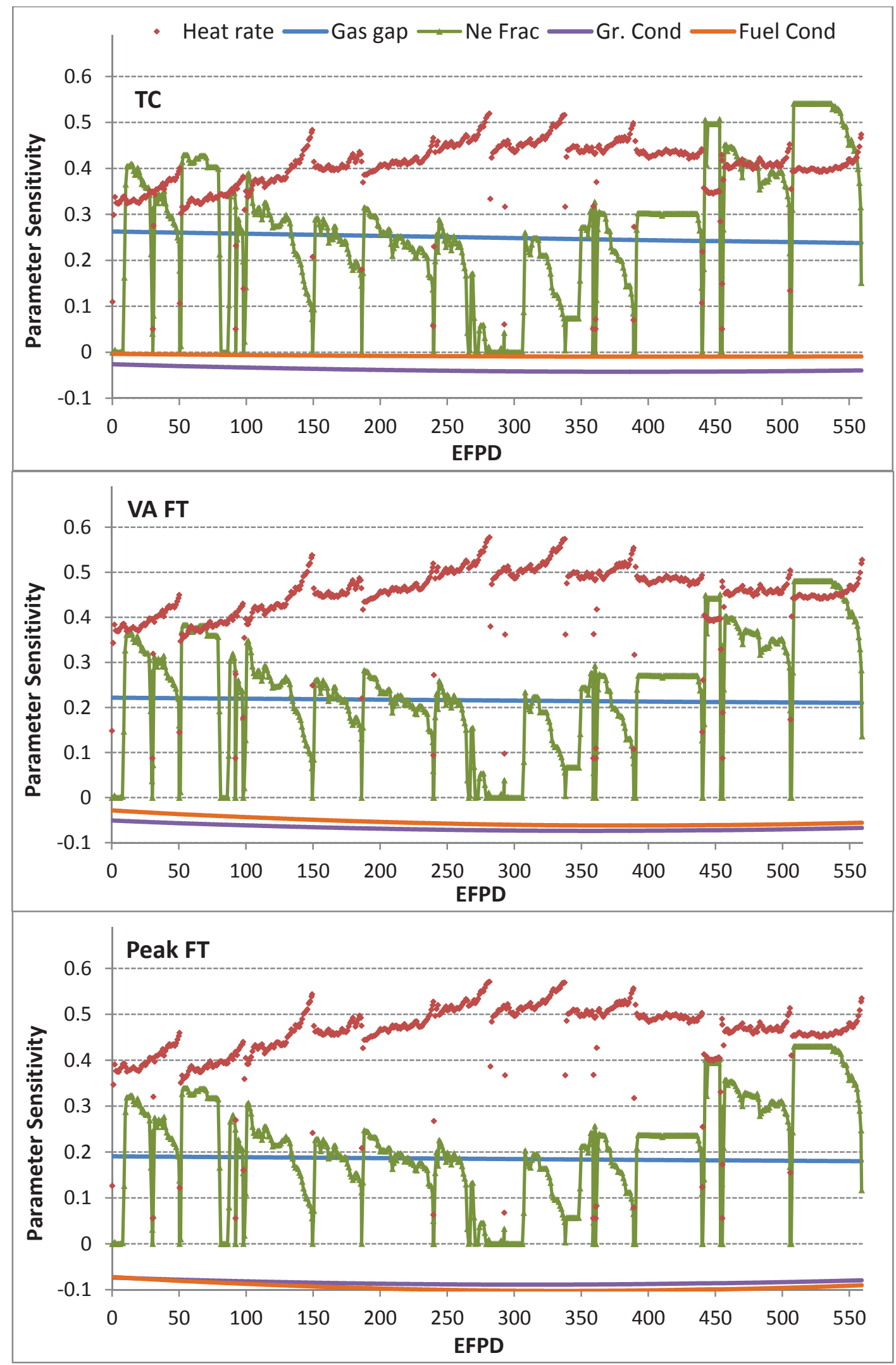

Figure 32. Daily parameter sensitivities for temperatures in Capsule 6. 


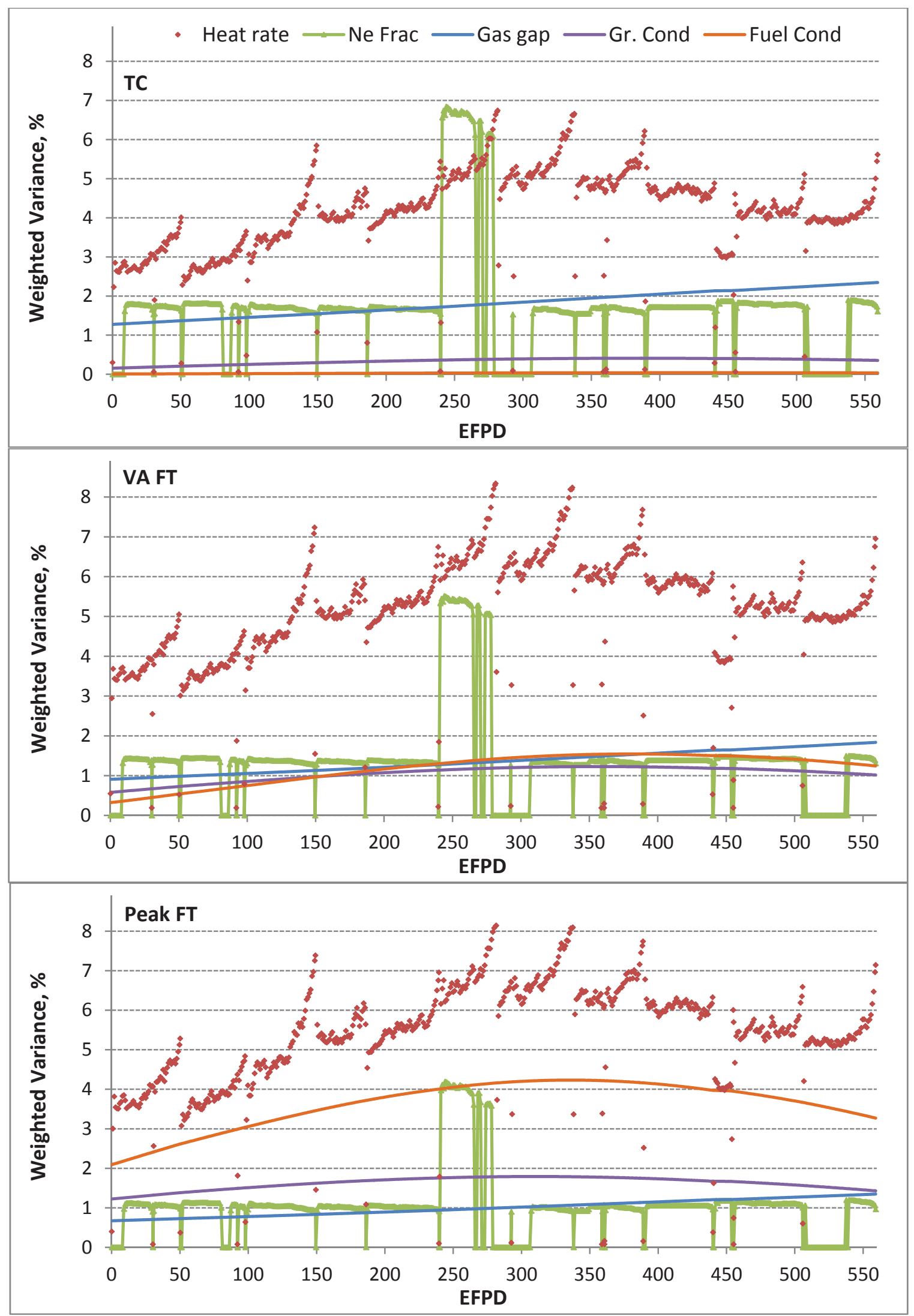

Figure 33. Daily temperature variances due to input uncertainties in Capsule 6. 


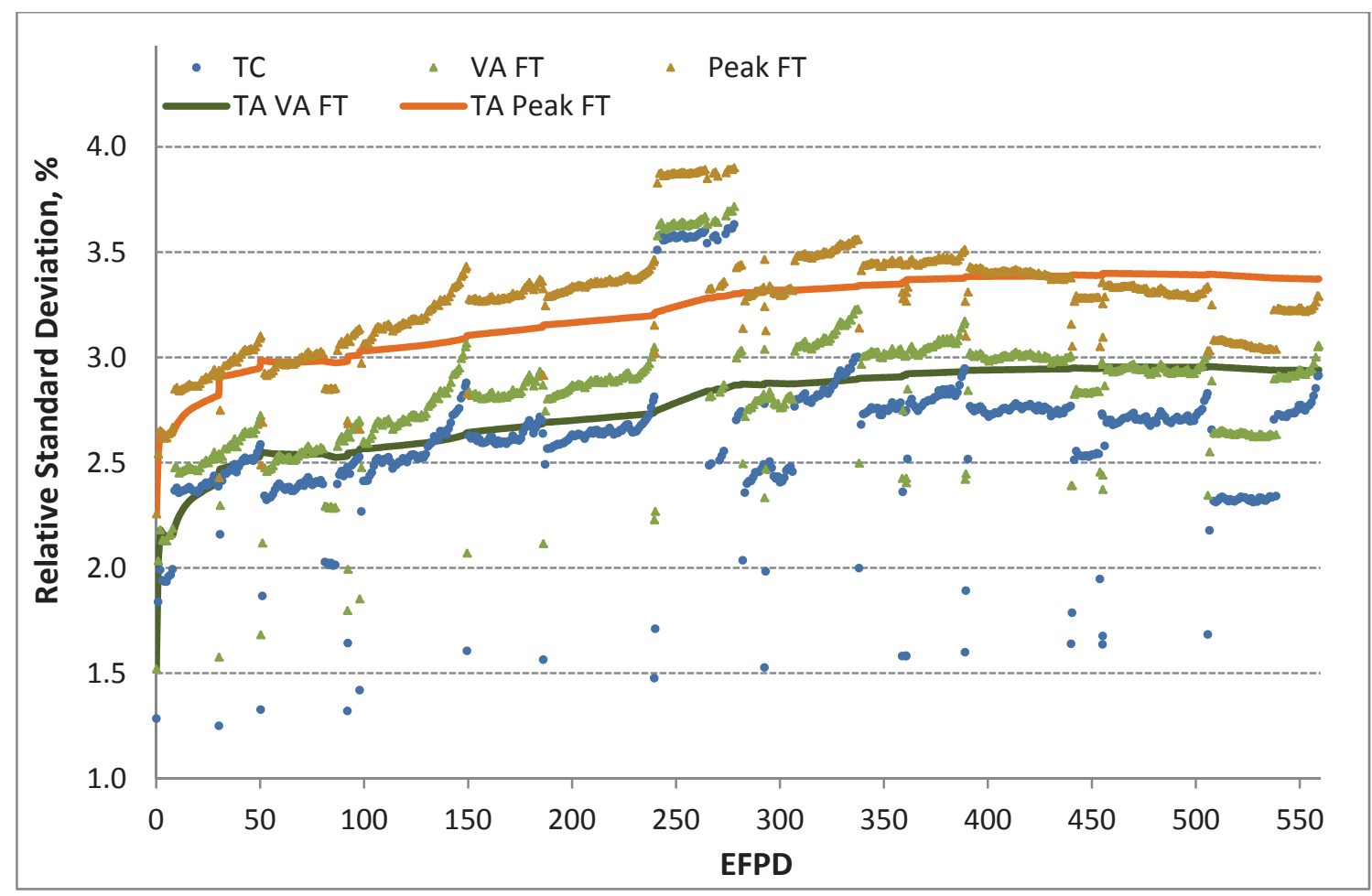

Figure 34. Capsule 6 temperature uncertainties in terms of relative standard deviations.

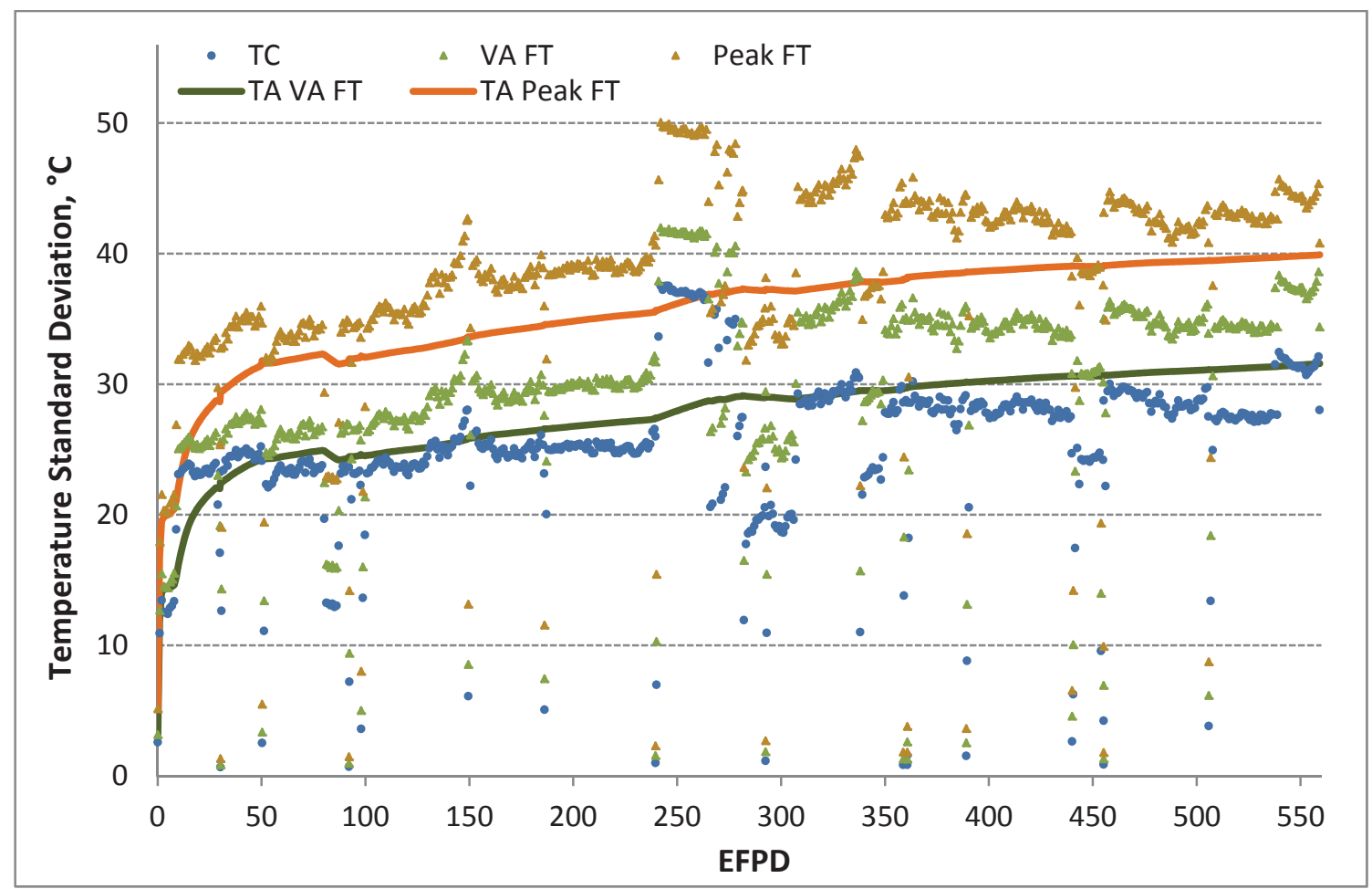

Figure 35. Daily standard deviations of predicted thermocouple and fuel temperatures in Capsule 6. 


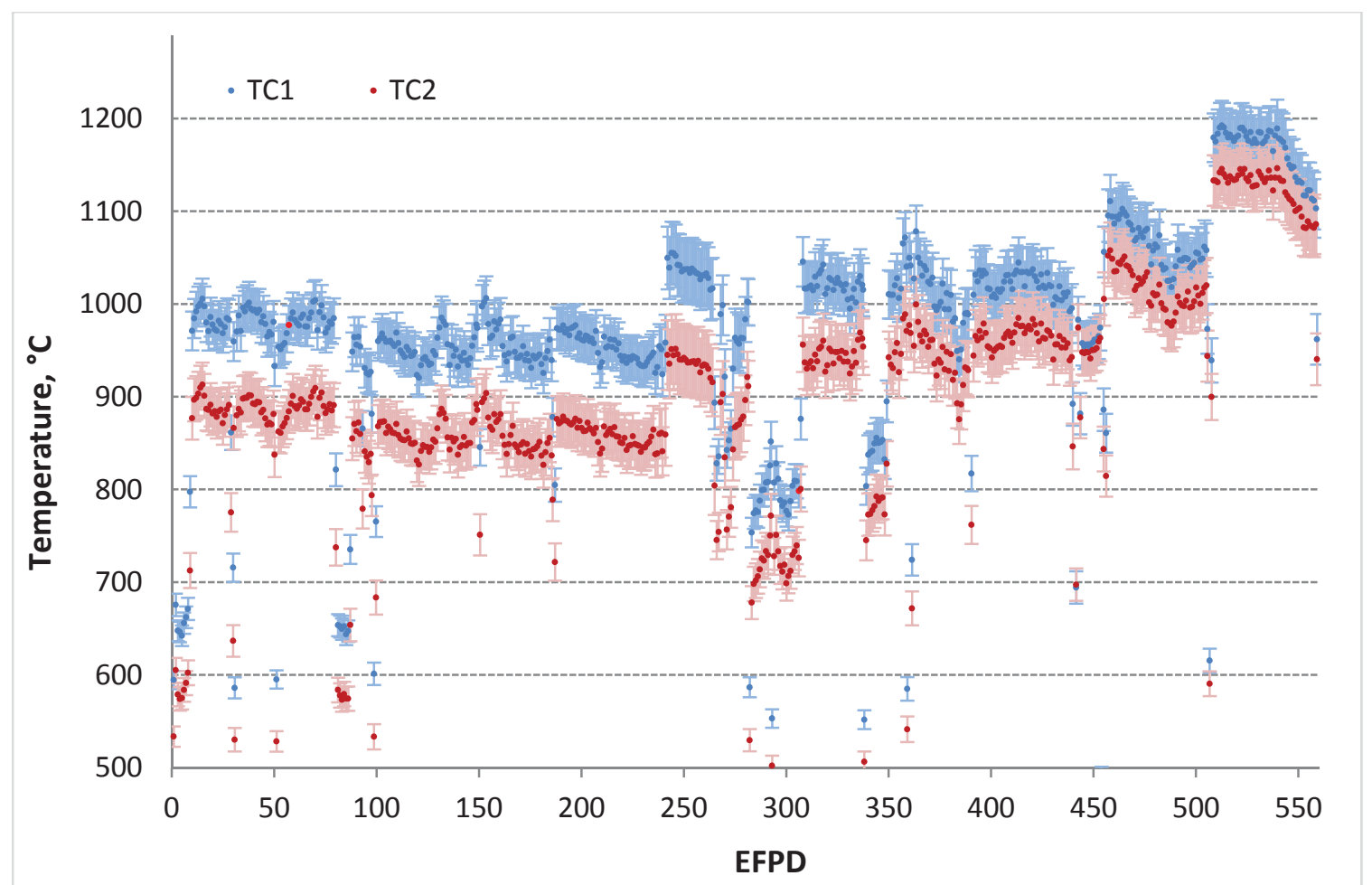

Figure 36. Model temperature and standard deviation of thermocouples in Capsule 6.

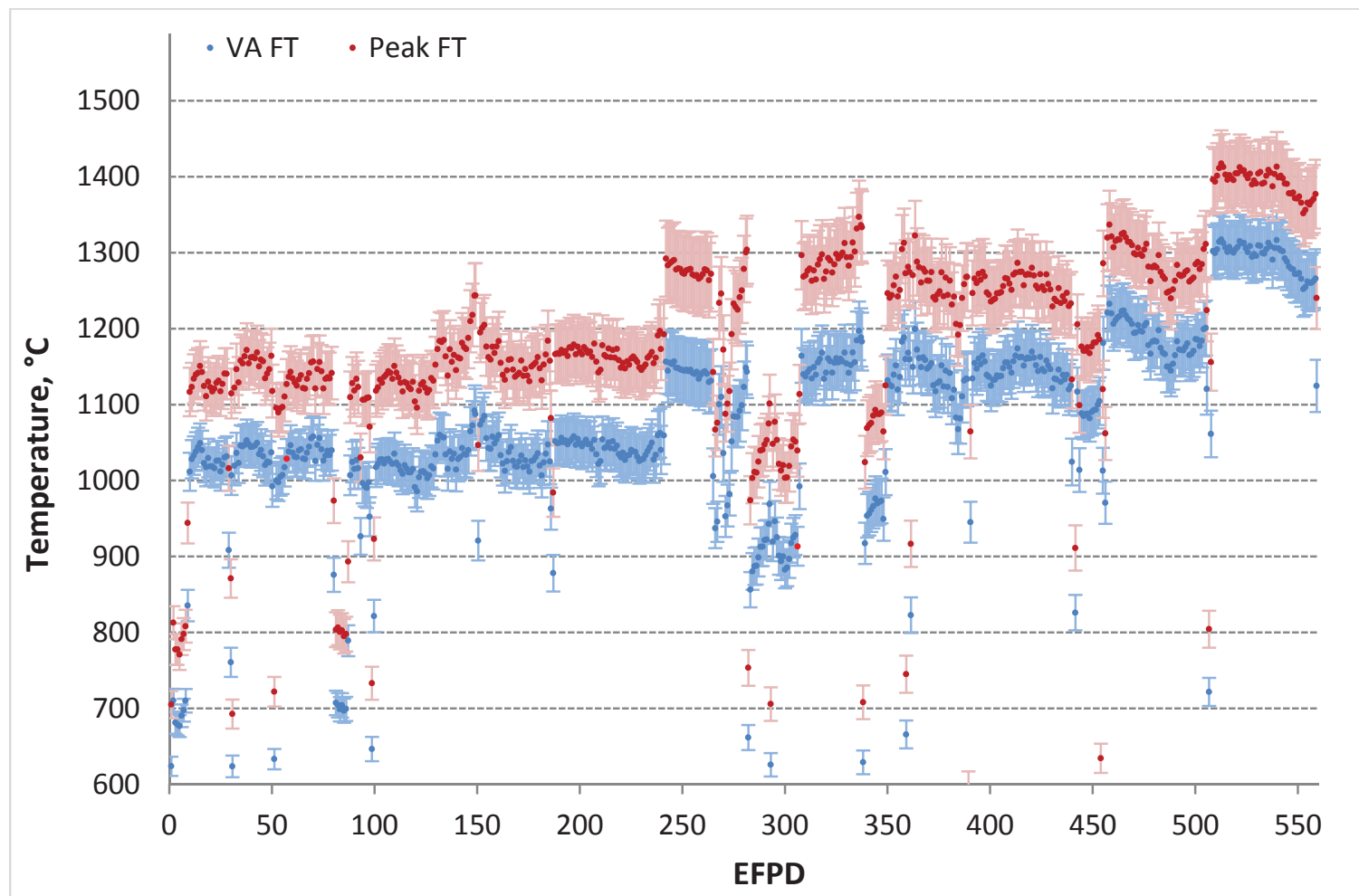

Figure 37. Model temperature and standard deviation of daily averaged fuel temperatures in Capsule 6. 


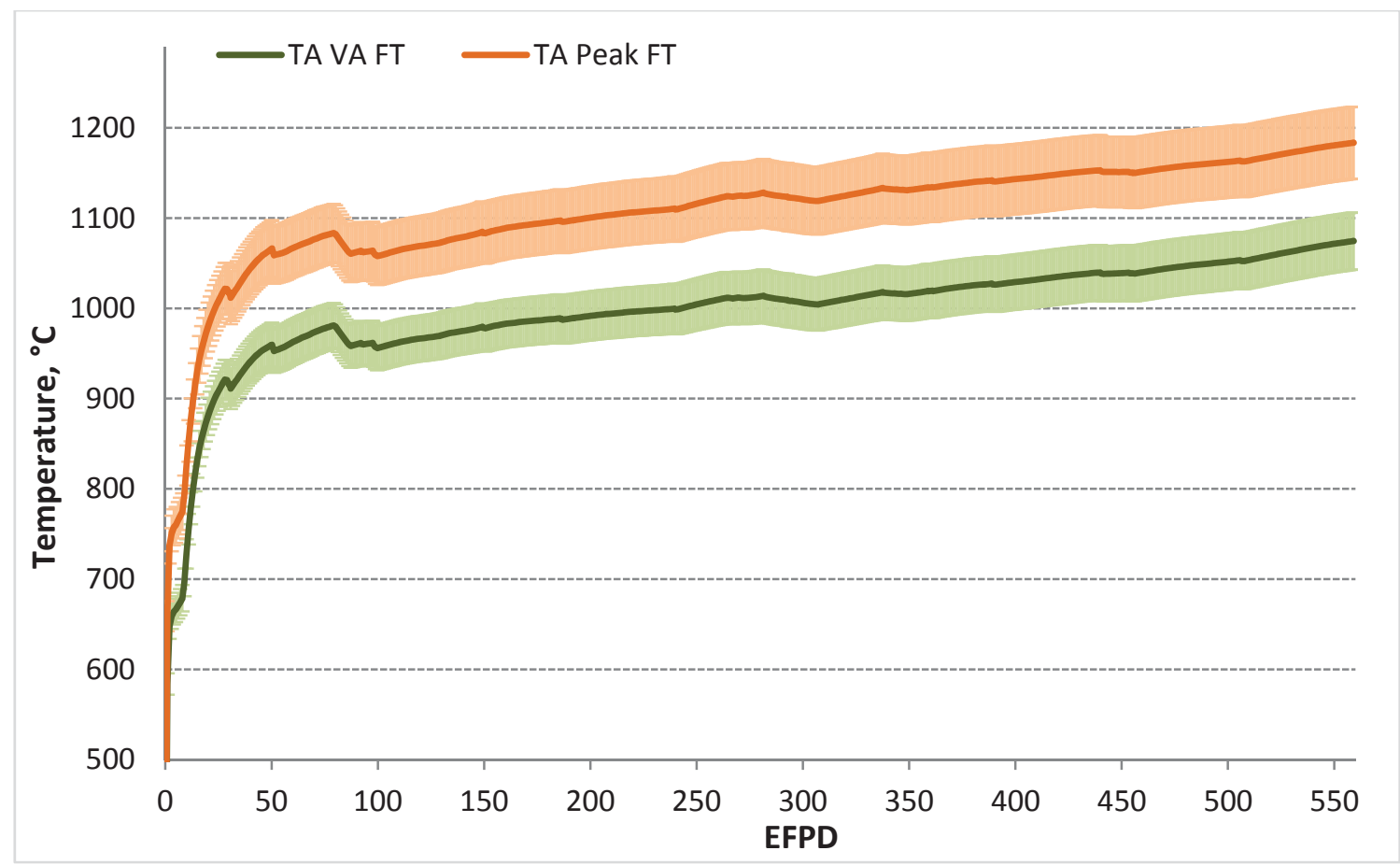

Figure 38. Model temperature and standard deviation of time-average fuel temperatures in Capsule 6.

\subsection{Temperature Uncertainty Results for Capsule 5}

Table 9 summarizes the results for Capsule 5. The overall uncertainties in terms of standard deviation are given both as a range over EFPDs for instantaneous temperatures and at the end of irradiation for time-average temperatures. Capsule 5 is the second to the top of the AGR-2 test train. The small control gap distance in this capsule leads to higher gap uncertainty and sensitivity, making control gap distance the dominant factor in TC temperature uncertainty. As discussed in Subsection 3.1.3.2. Capsule 5 may have model bias of $60^{\circ} \mathrm{C}$ (over prediction), based on the consistently negative TC residuals. The temperature uncertainty results due to input uncertainties specific for Capsule 5 are listed below:

- Input parameter uncertainty (Figure 39): The uncertainties of fuel heat rate and fuel and graphite thermal conductivities are assumed constant over the entire irradiation, and the relative uncertainties of neon fraction (green triangles) are similar to Capsule 6. Capsule 5 has the smallest control gap distance, which leads to the greatest gap uncertainties ranging from $6.5 \%$ to $9.7 \%$.

- Input parameter sensitivity (Figure 40): The fuel heat rate sensitivities (red dots) are the greatest for most days of irradiation for all temperatures, and the sensitivities of fuel and graphite thermal conductivities (orange and purple lines) are the smallest, especially for temperatures at peripheral TC location (top plot). The smaller control gap in Capsule 5 leads to an increase in the gap sensitivity relative to Capsule 6.

- Weighted temperature variance (Figure 41 ): The 5\% error in the fuel heat rate is only a dominant factor for VA and peak FT uncertainties. The greater control gap uncertainty and sensitivity causes the gap distance to become a dominant factor for TC temperature uncertainty (blue line on the top plot of Figure 41). 
- Overall calculated temperature uncertainty: The daily relative (in \%) and absolute (in ${ }^{\circ} \mathrm{C}$ ) $\mathrm{TC}$ and fuel compact temperature model uncertainties in terms of standard deviation are presented in Figure 42 and Figure 43. The overall uncertainties of peak FT (light-brown triangles) are as large as $4.2 \%$ (or $60^{\circ} \mathrm{C}$ ). For temperatures at TCs, the overall uncertainty ranged from $3.3 \%$ to $4.5 \%$ (highest). This highest TC uncertainty is caused mainly by the high uncertainty of the small control gas gap distance at the same time as high neon fraction uncertainty due to gas line cross-talk failure.

The daily temperature and one standard deviation of the predicted temperatures in Capsule 5 are plotted for peripheral TC1 and TC2 in Figure 44, daily average VA FT and daily peak FT in Figure 45, and TA VA FT and TA peak FT in Figure 46.

Table 9. Summary of temperature uncertainty results for Capsule 5.

\begin{tabular}{|l|c|c|c|c|c|}
\hline & TC & VA FT & Peak FT & $\begin{array}{c}\text { TA VA FT } \\
\text { at EOE }\end{array}$ & $\begin{array}{c}\text { TA Peak } \\
\text { FT at EOE }\end{array}$ \\
\hline \multicolumn{1}{|c|}{ Dominant parameter } & $\begin{array}{c}\text { Control } \\
\text { gas gap }\end{array}$ & $\begin{array}{c}\text { Fuel } \\
\text { heat rate }\end{array}$ & $\begin{array}{c}\text { Fuel } \\
\text { heat rate }\end{array}$ & & \\
\hline Dominant sensitivity & $0.32-0.39$ & $0.42-0.60$ & $0.44-0.56$ & & \\
\hline $\begin{array}{l}\text { Dominant weighted } \\
\text { variance, \% }\end{array}$ & $6.0-9.2$ & $4.6-8.7$ & $4.7-8.1$ & & \\
\hline Relative uncertainty, \% & $3.3-4.5$ & $3.0-4.2$ & $3.3-4.2$ & 3.5 & 3.7 \\
\hline Absolute uncertainty, ${ }^{\circ} \mathrm{C}$ & $30-45$ & $32-52$ & $38-60$ & 39 & 45 \\
\hline
\end{tabular}

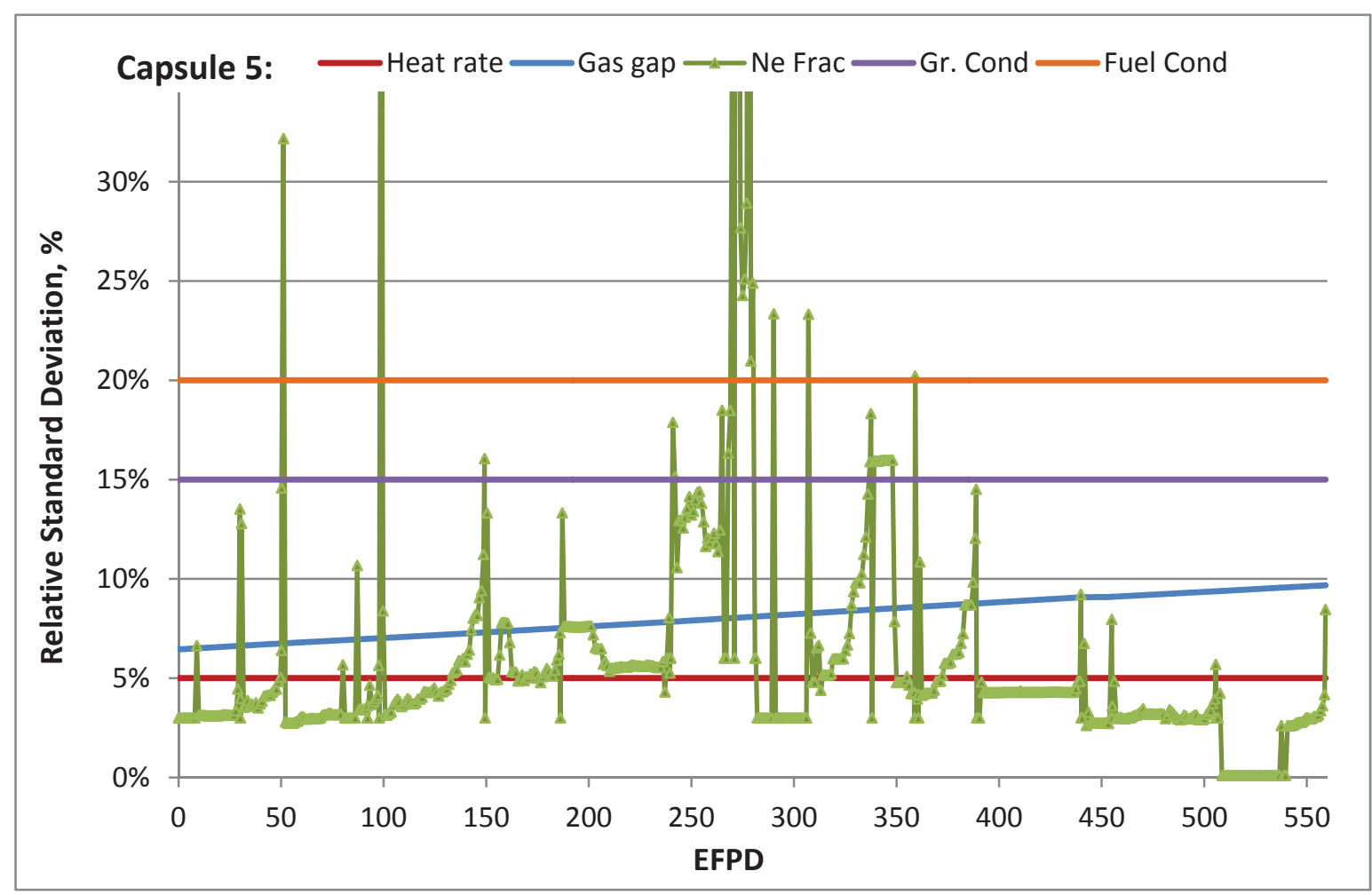

Figure 39. Daily input relative standard deviations for Capsule 5. 


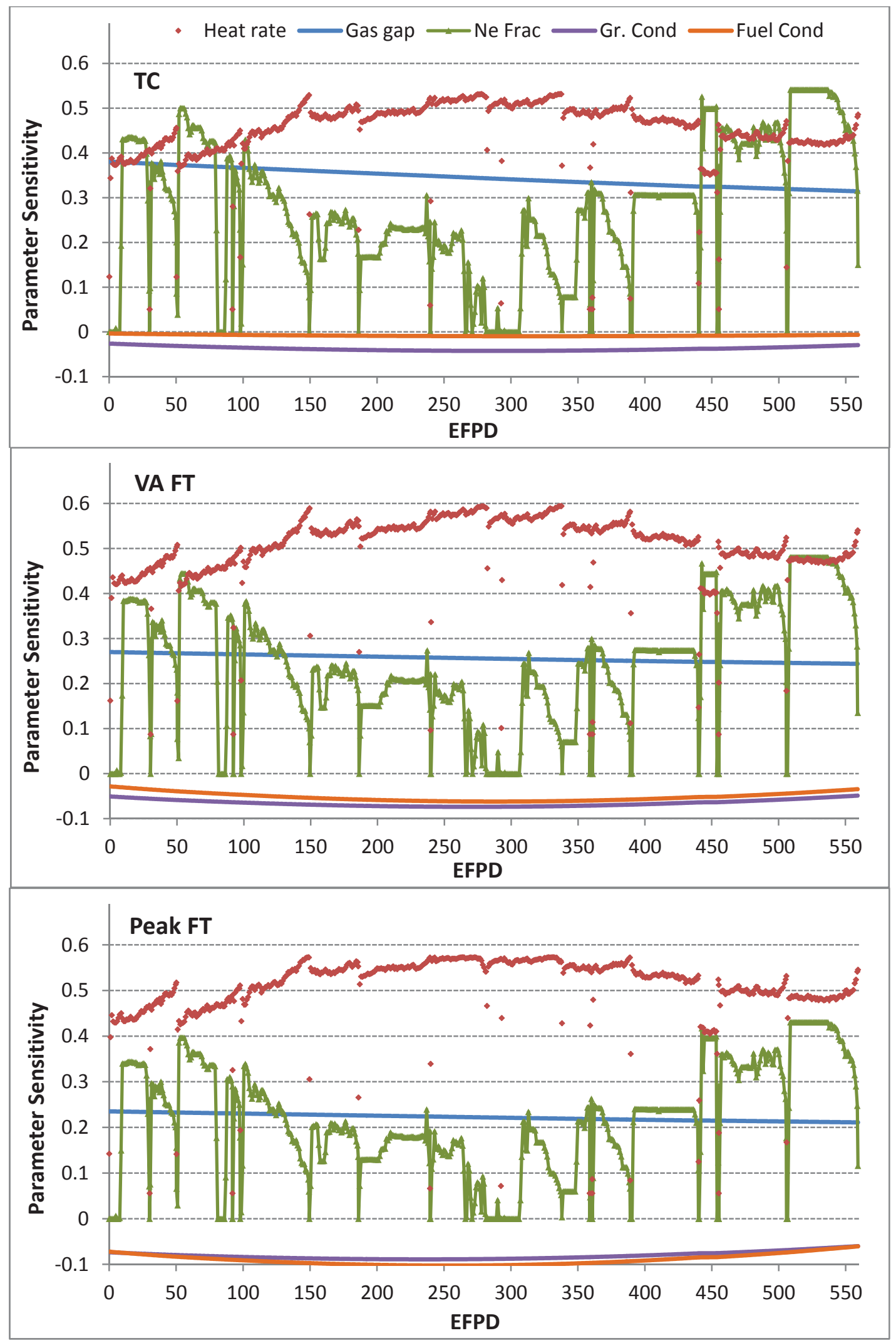

Figure 40. Daily parameter sensitivities for temperatures in Capsule 5. 


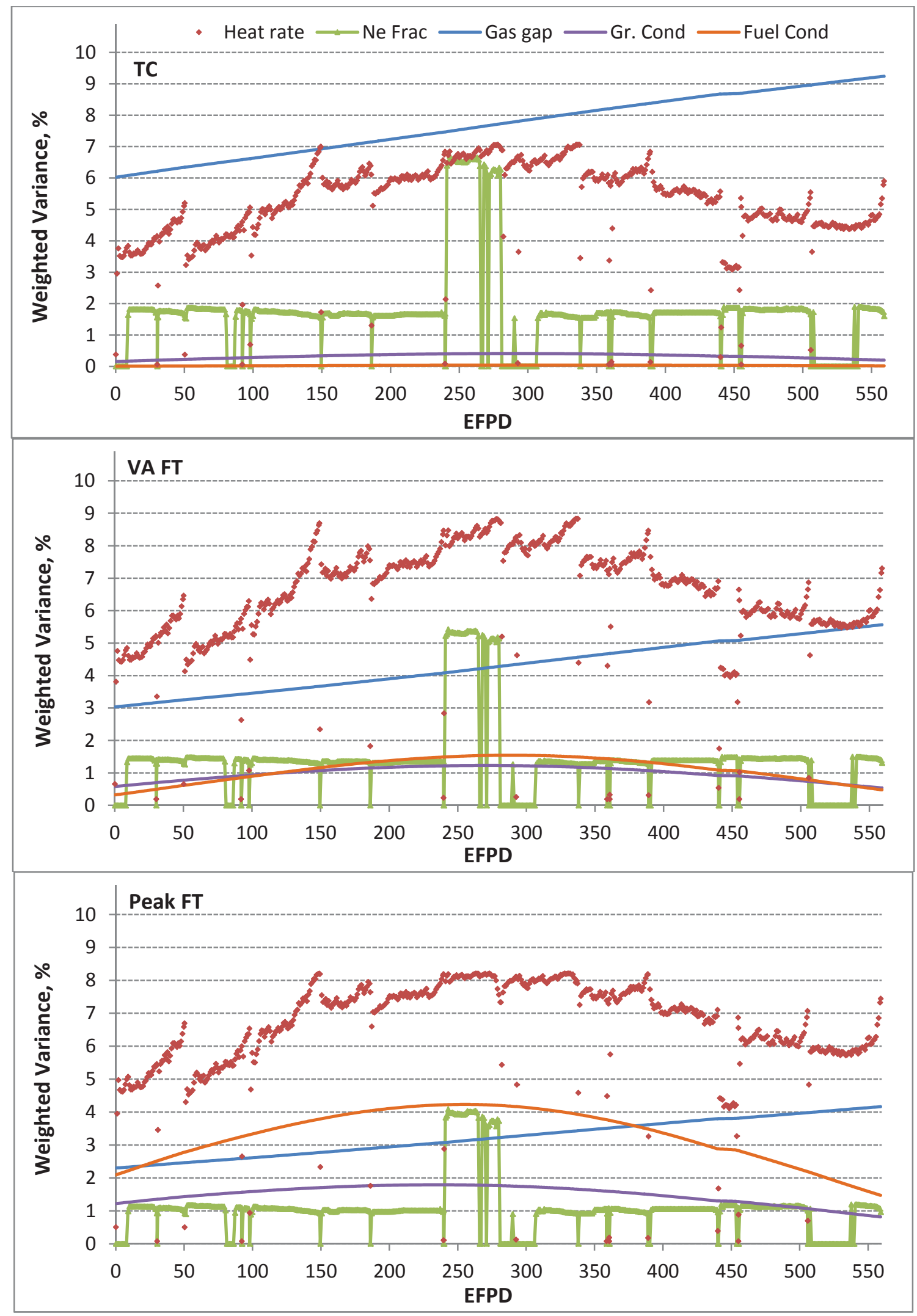

Figure 41. Daily temperature variances due to input uncertainties in Capsule 5. 


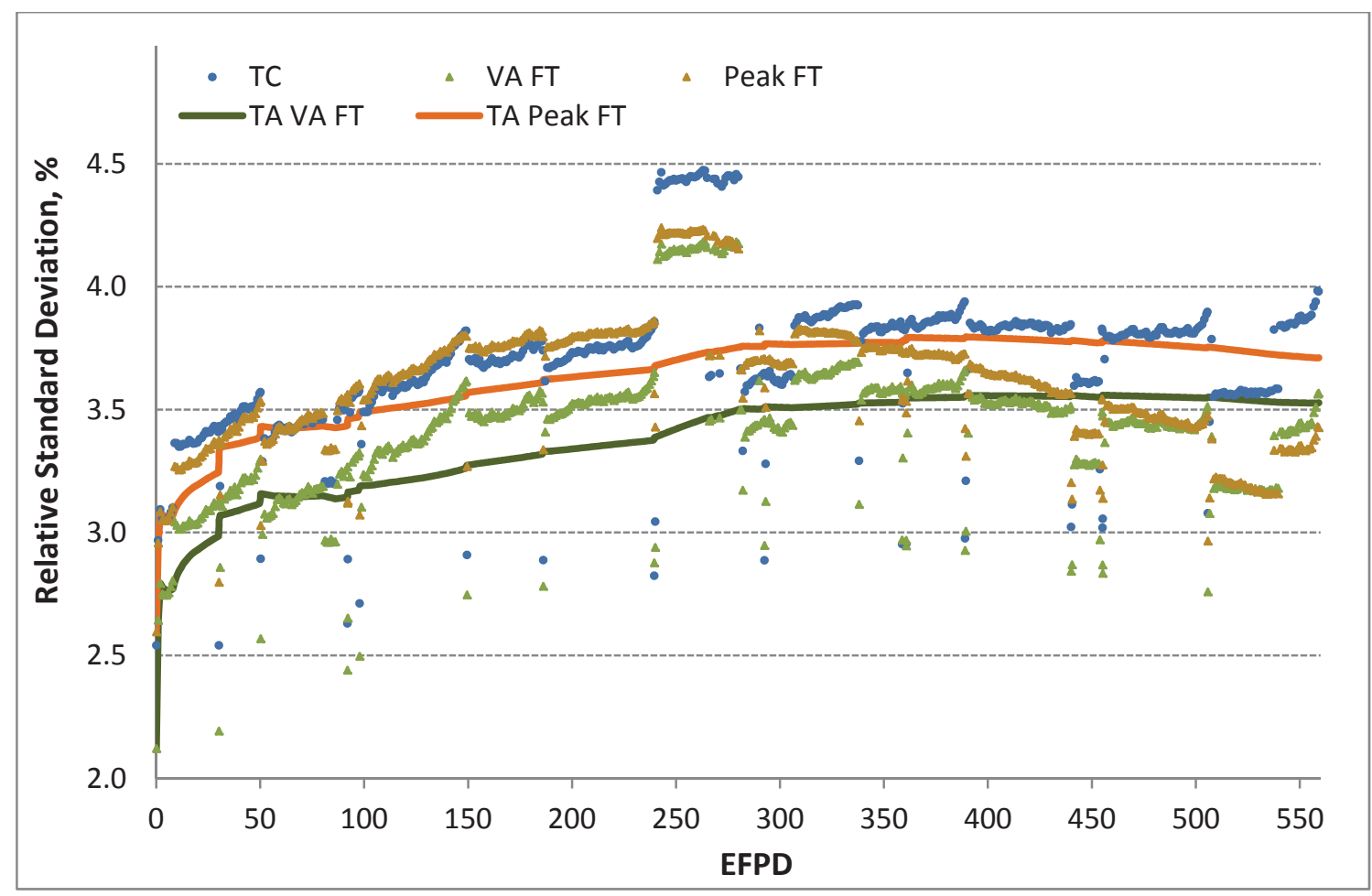

Figure 42. Capsule 5 temperature uncertainties in terms of relative standard deviations.

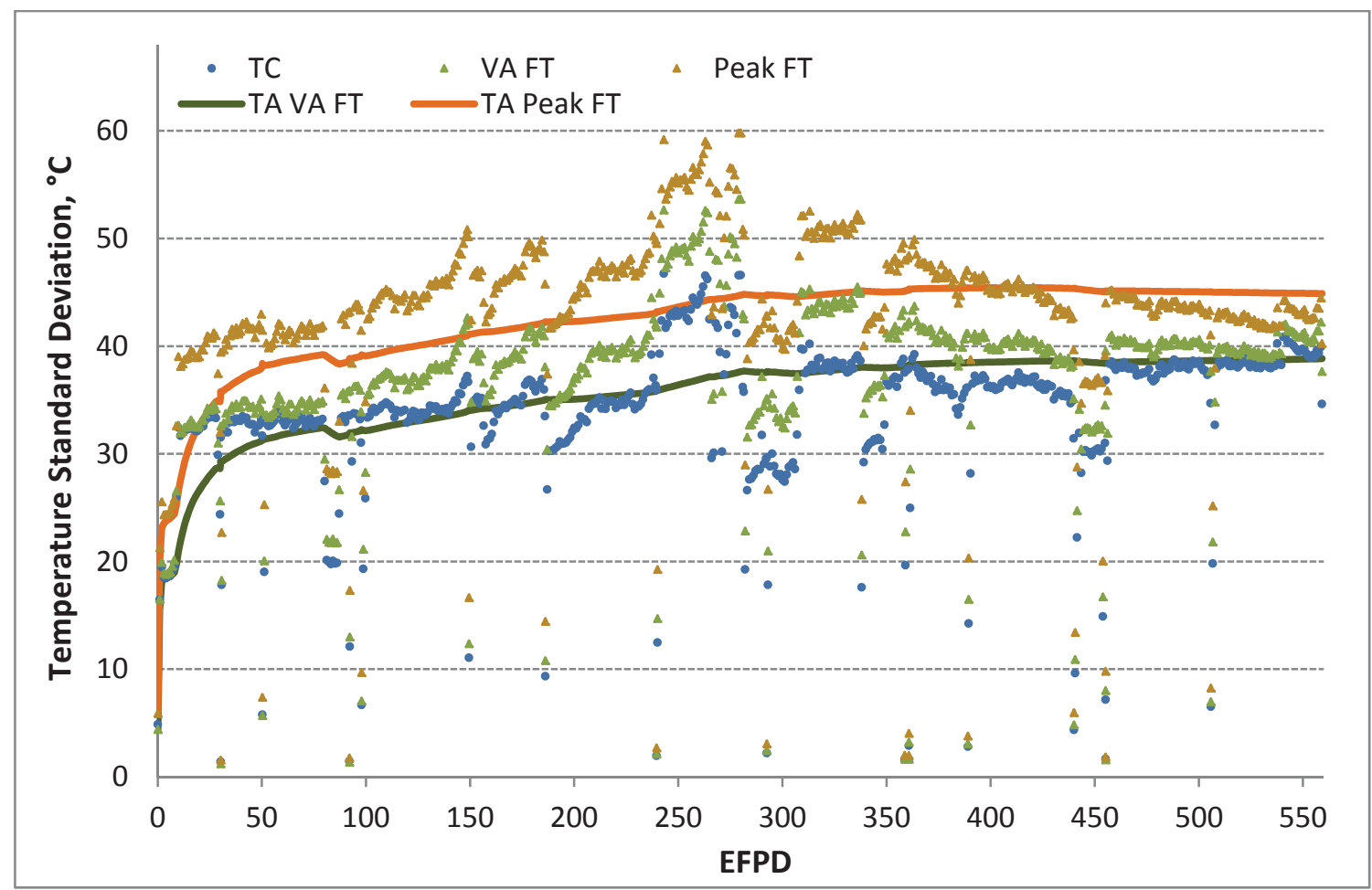

Figure 43. Daily standard deviations of predicted thermocouple and fuel temperatures in Capsule 5. 


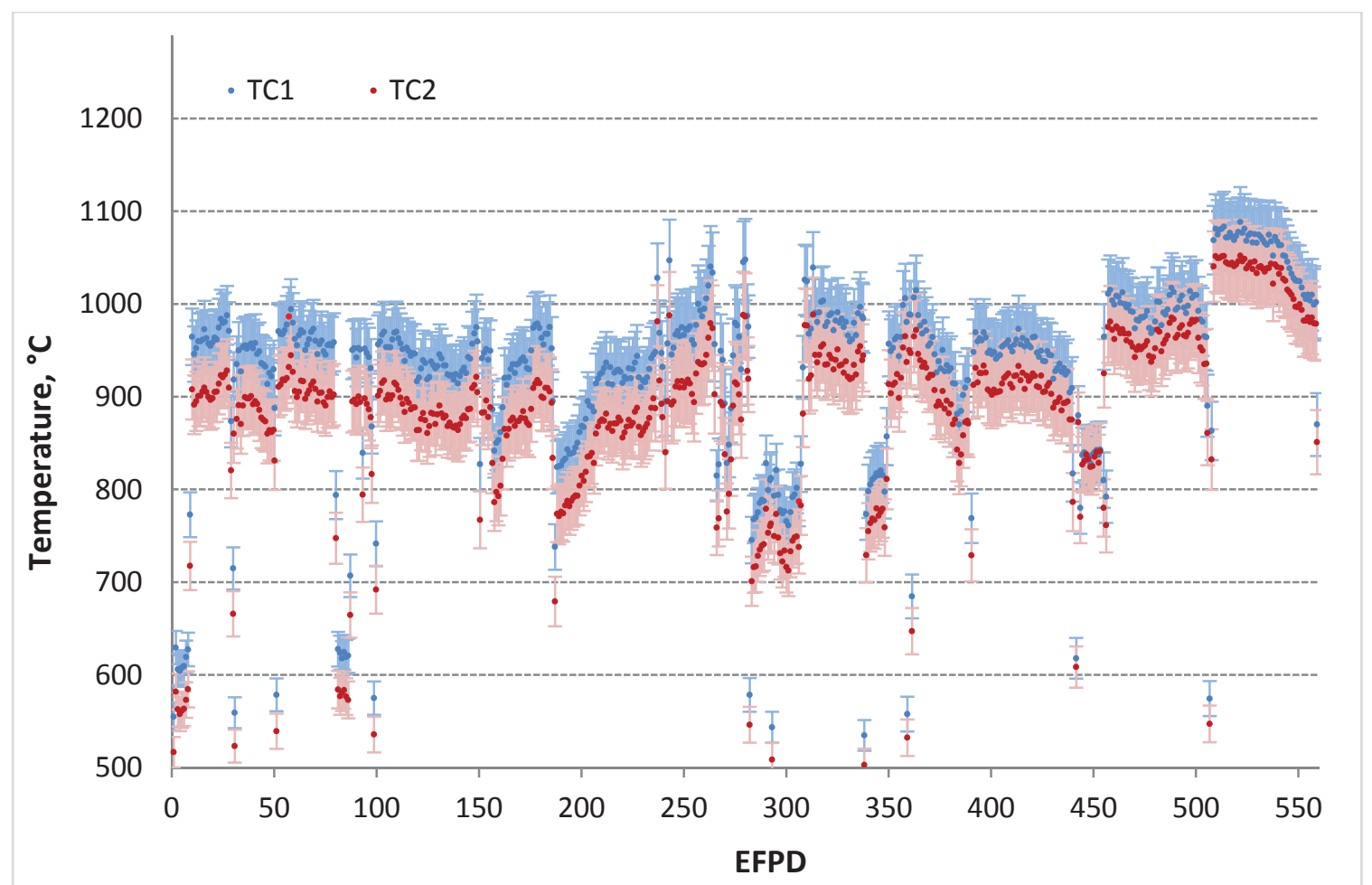

Figure 44. Model temperature and standard deviation of thermocouples in Capsule 5.

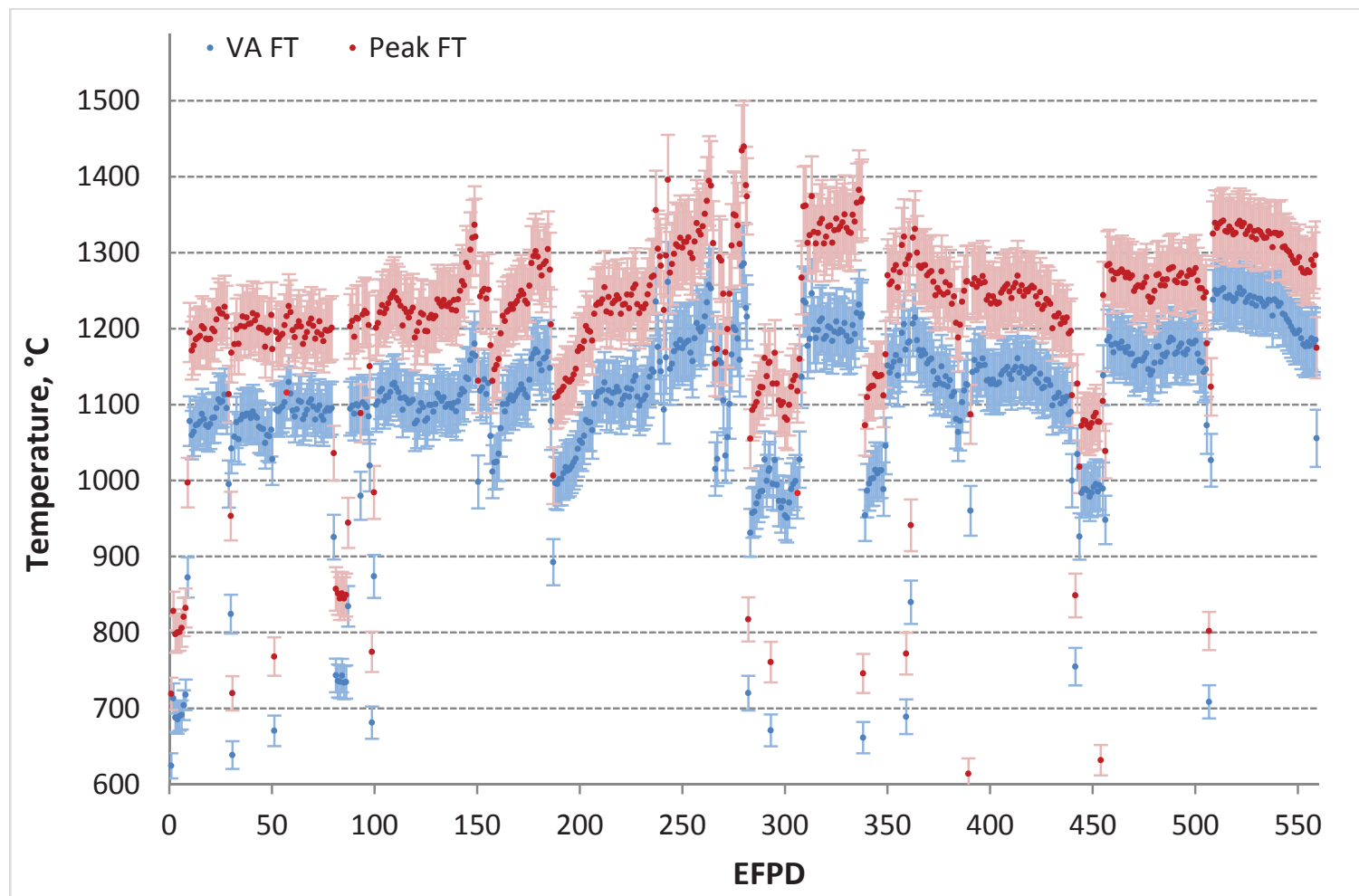

Figure 45. Model temperature and standard deviation of daily averaged fuel temperatures in Capsule 5. 


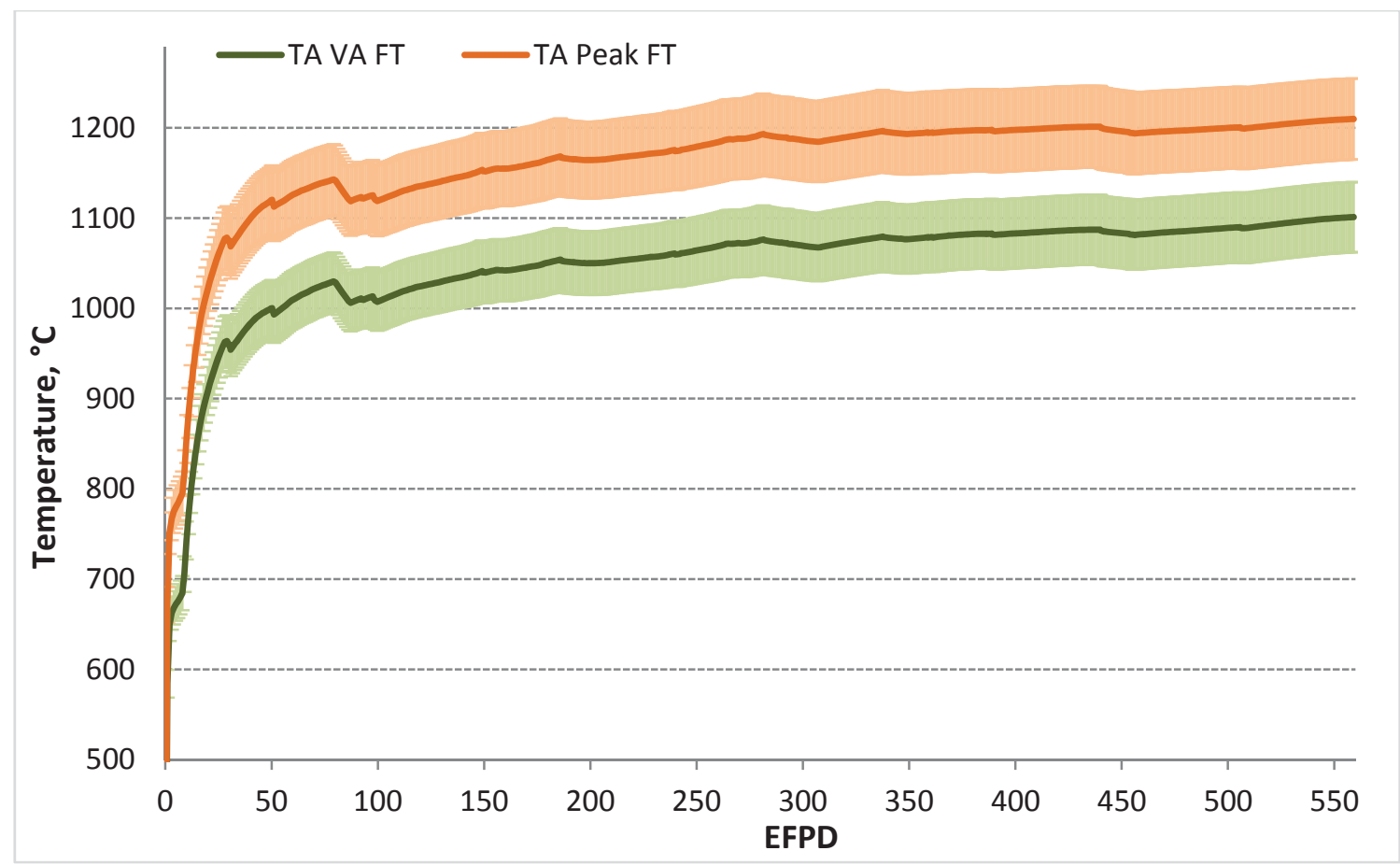

Figure 46. Model temperature and standard deviation of time-average fuel temperatures in Capsule 5.

\subsection{Temperature Uncertainty Results for Capsule 3}

Table 10 summarizes the results for Capsule 3. Capsule 3 is located in the middle of the AGR-2 test train. The largest control gap distance in this capsule leads to the lowest gap uncertainty and sensitivity, which helps reduce the overall Capsule 3 temperature uncertainties. Temperature uncertainty results specific to Capsule 3 are listed below:

- Input parameter uncertainty (Figure 47): The uncertainties of fuel heat rate and fuel and graphite thermal conductivities are assumed constant over the entire irradiation, and the relative uncertainties of neon fraction (green triangles) are similar to Capsule 6. Capsule 3 has the biggest control gap distance, which leads to the lowest gap uncertainties, ranging from $3.2 \%$ to $4.8 \%$.

- Input parameter sensitivity (Figure 48): The fuel heat rate sensitivities (red dots) are the greatest for most days of irradiation for all temperatures and the sensitivities of fuel thermal conductivity (orange lines) are smallest, especially for temperature at peripheral TC location (top plot). The absolute value of the graphite thermal conductivity sensitivity (purple lines) is slightly higher than the fuel conductivity sensitivity for TC and VA FT temperature. They are almost the same for peak FT. The large control gap in Capsule 3 leads to gap sensitivity less than 0.2 .

- Weighted temperature variance (Figure 49): The 5\% error in the fuel heat rate is a dominant factor for all calculated temperature uncertainties (TCs, VA FT, and peak FT).

- Overall calculated temperature uncertainty: The daily relative (in \%) and absolute (in ${ }^{\circ} \mathrm{C}$ ) TC and fuel compact temperature model uncertainties in terms of standard deviation are presented in Figure 50 and Figure 51. The overall uncertainties of peak FT (light-brown triangles) are the highest up to $3.7 \%$ (or $45^{\circ} \mathrm{C}$ ), which are lowest among the four U.S. AGR-2 capsules.

The daily temperature and one standard deviation of the predicted temperatures in Capsule 3 are plotted for peripheral TC1 and TC2 in Figure 52, daily average VA FT, and daily peak FT in Figure 53; and TA VA FT and TA peak FT in Figure 54. 
Table 10. Summary of temperature uncertainty results for Capsule 3.

\begin{tabular}{|l|c|c|c|c|c|}
\hline & TC & VA FT & Peak FT & $\begin{array}{c}\text { TA VA FT } \\
\text { at EOE }\end{array}$ & $\begin{array}{c}\text { TA Peak } \\
\text { FT at EOE }\end{array}$ \\
\hline \multicolumn{1}{|c|}{ Dominant parameter } & $\begin{array}{c}\text { Fuel } \\
\text { Heat Rate }\end{array}$ & $\begin{array}{c}\text { Fuel } \\
\text { Heat Rate }\end{array}$ & $\begin{array}{c}\text { Fuel } \\
\text { Heat Rate }\end{array}$ & & \\
\hline Dominant sensitivity & $0.30-0.50$ & $0.34-0.55$ & $0.34-0.55$ & & \\
\hline $\begin{array}{l}\text { Dominant weighted } \\
\text { variance, \% }\end{array}$ & $2.0-6.3$ & $3.0-8.0$ & $3.0-8.0$ & & \\
\hline Relative uncertainty, \% & $2.1-3.4$ & $2.2-3.5$ & $2.7-3.7$ & 2.7 & 3.1 \\
\hline Absolute uncertainty, ${ }^{\circ} \mathrm{C}$ & $17-33$ & $21-40$ & $27-45$ & 28 & 34 \\
\hline
\end{tabular}

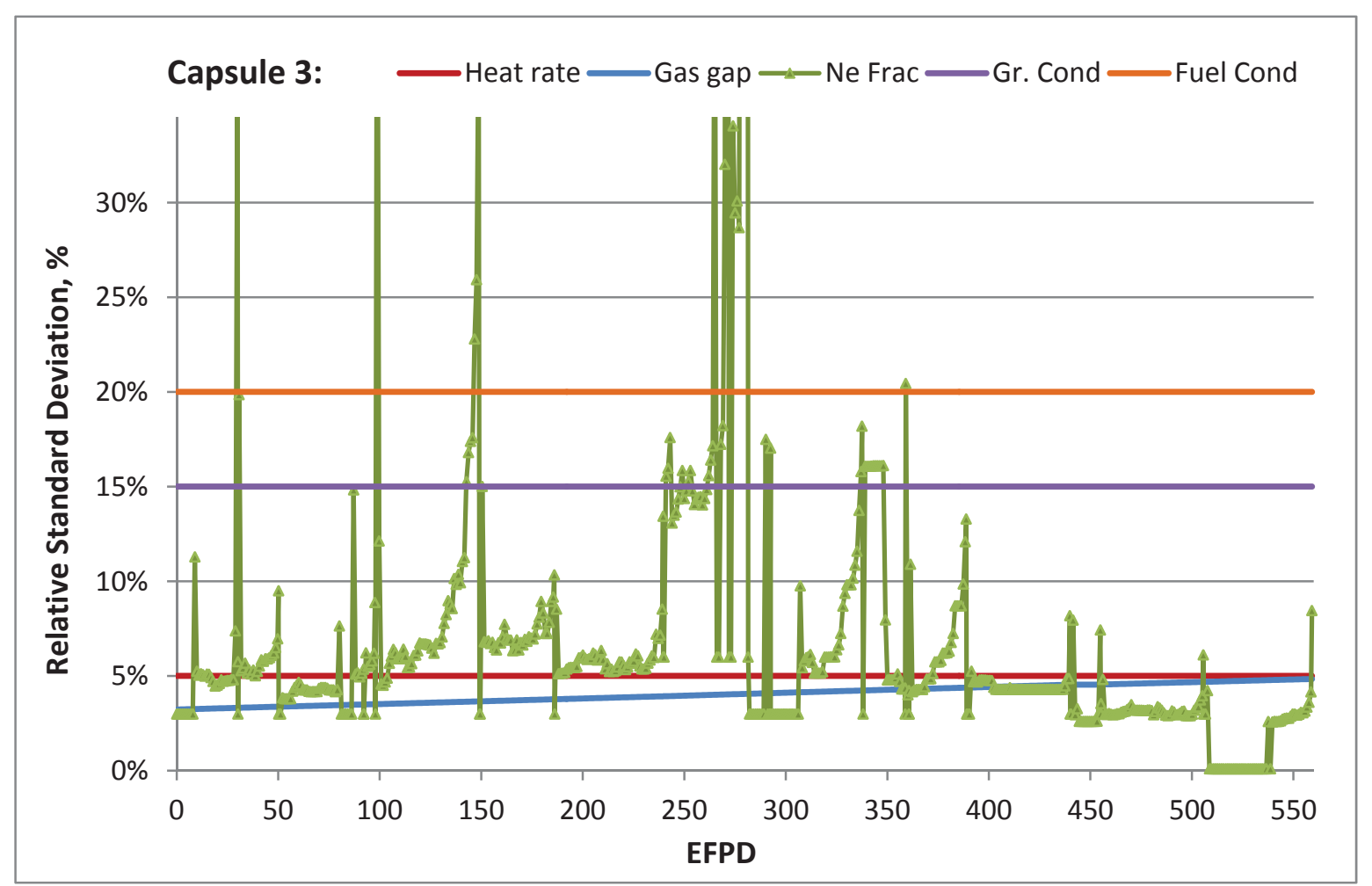

Figure 47. Daily input relative standard deviations for Capsule 3. 


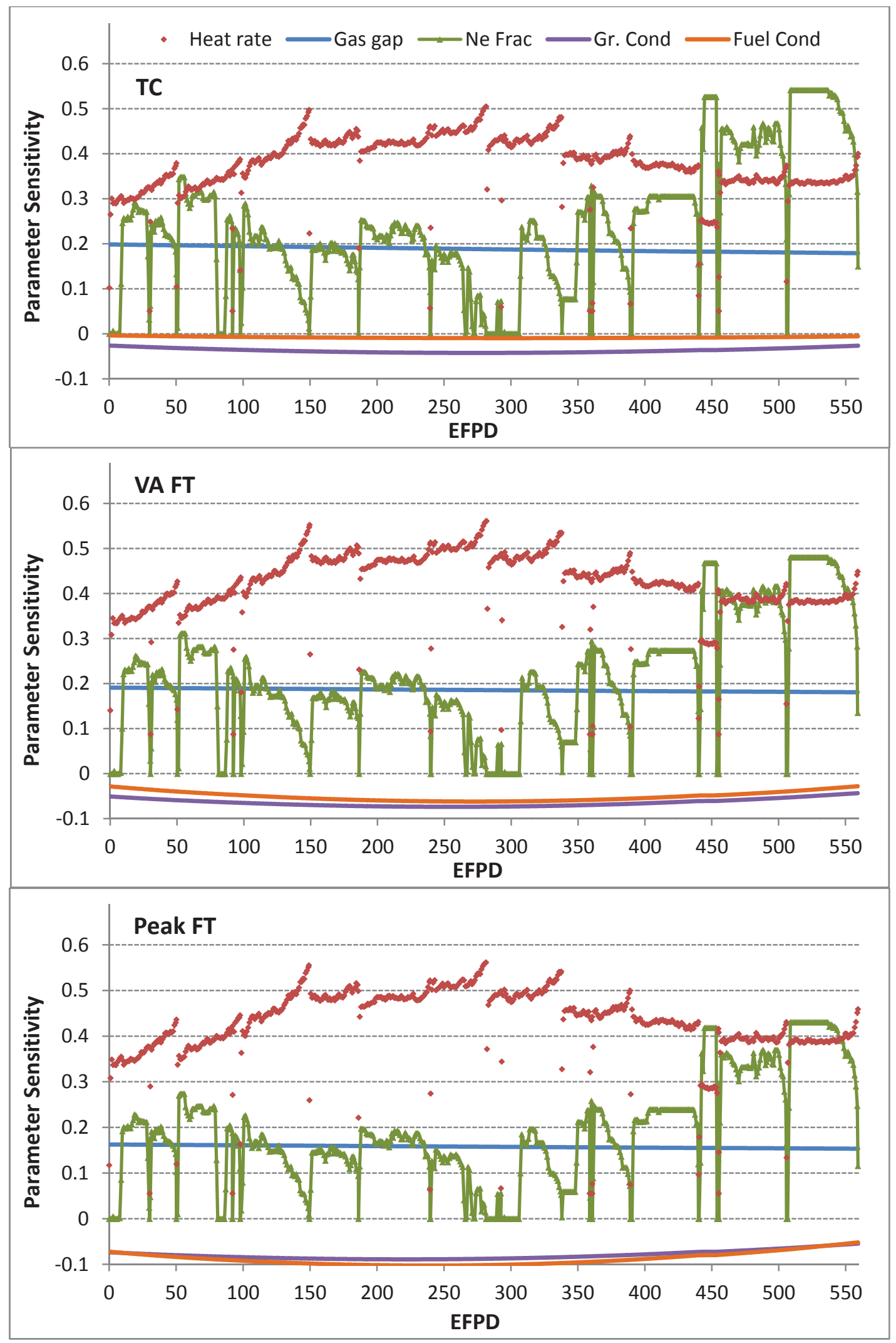

Figure 48. Daily parameter sensitivities for temperatures in Capsule 3. 


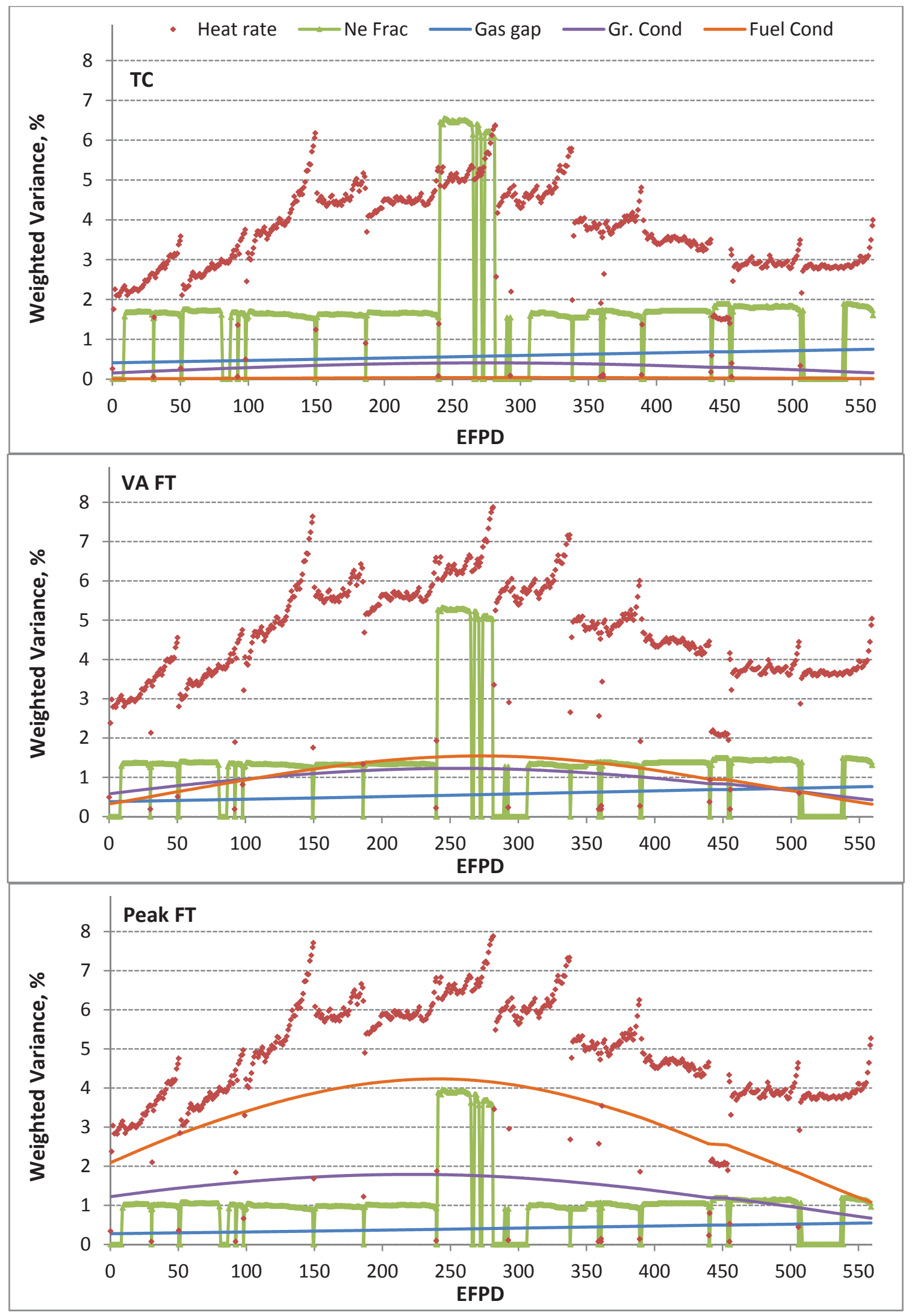

Figure 49. Daily temperature variances due to input uncertainties in Capsule 3. 


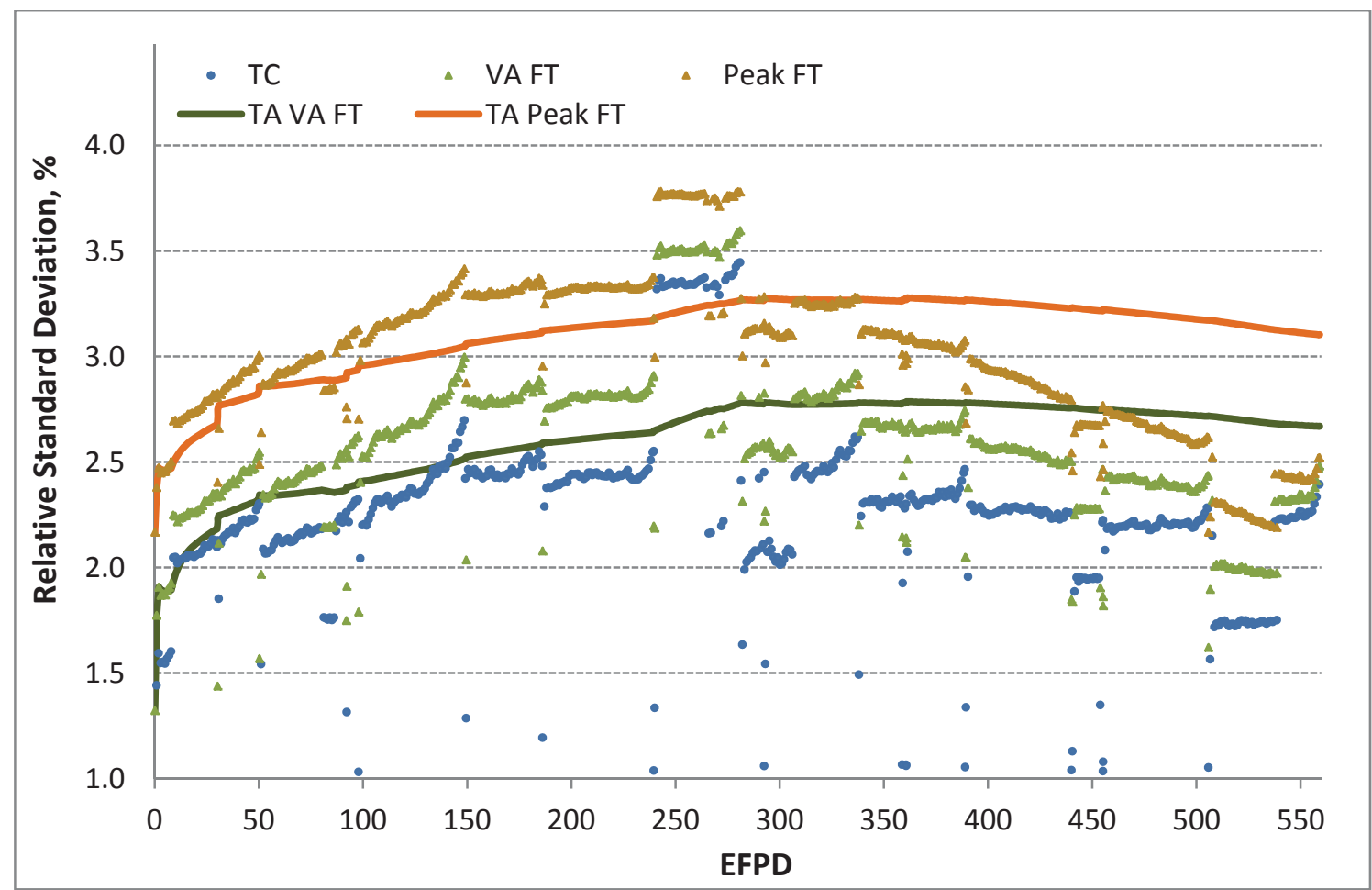

Figure 50. Capsule 3 temperature uncertainties in terms of relative standard deviations.

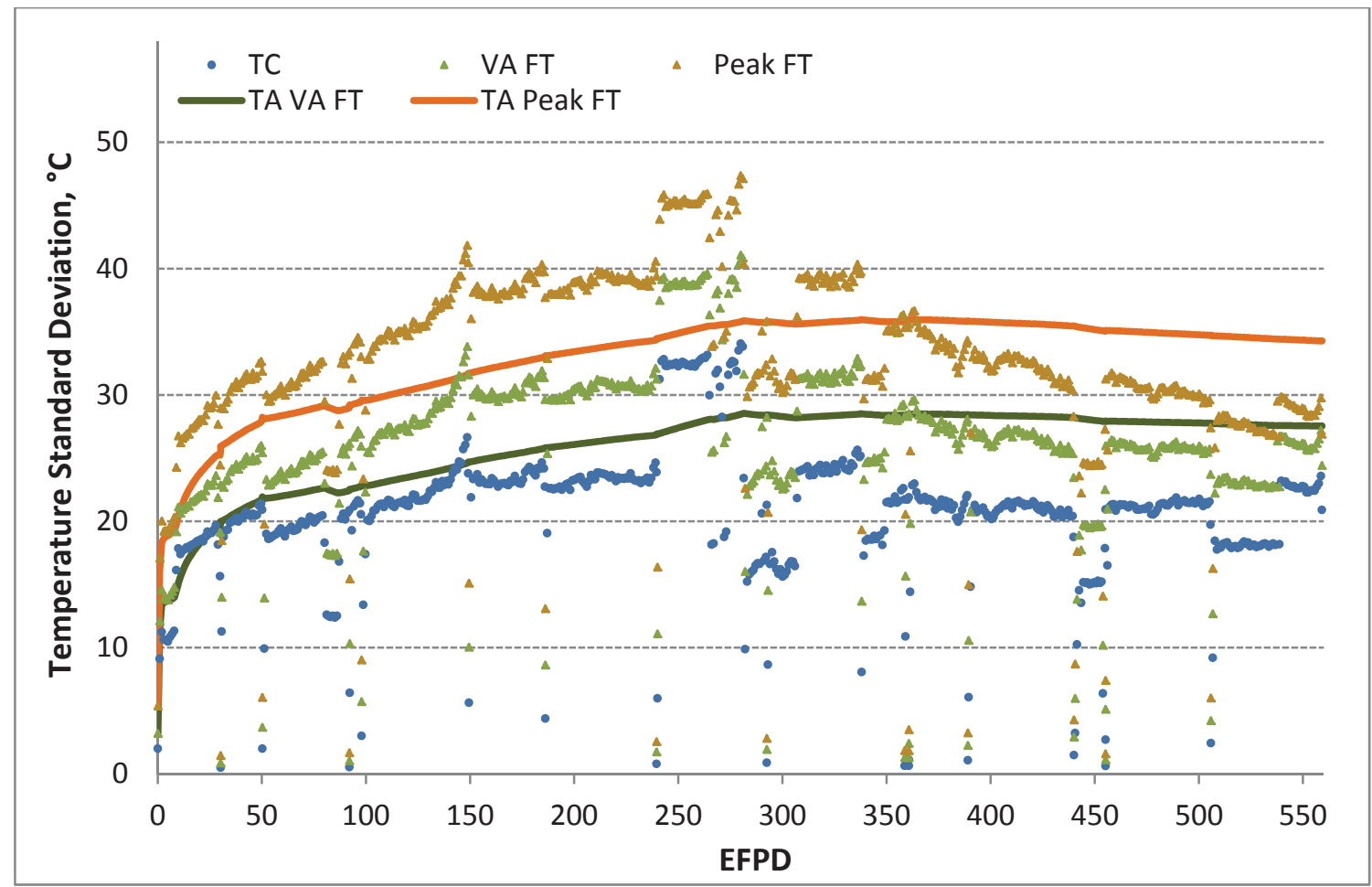

Figure 51. Daily standard deviations of predicted thermocouple and fuel temperatures in Capsule 3. 


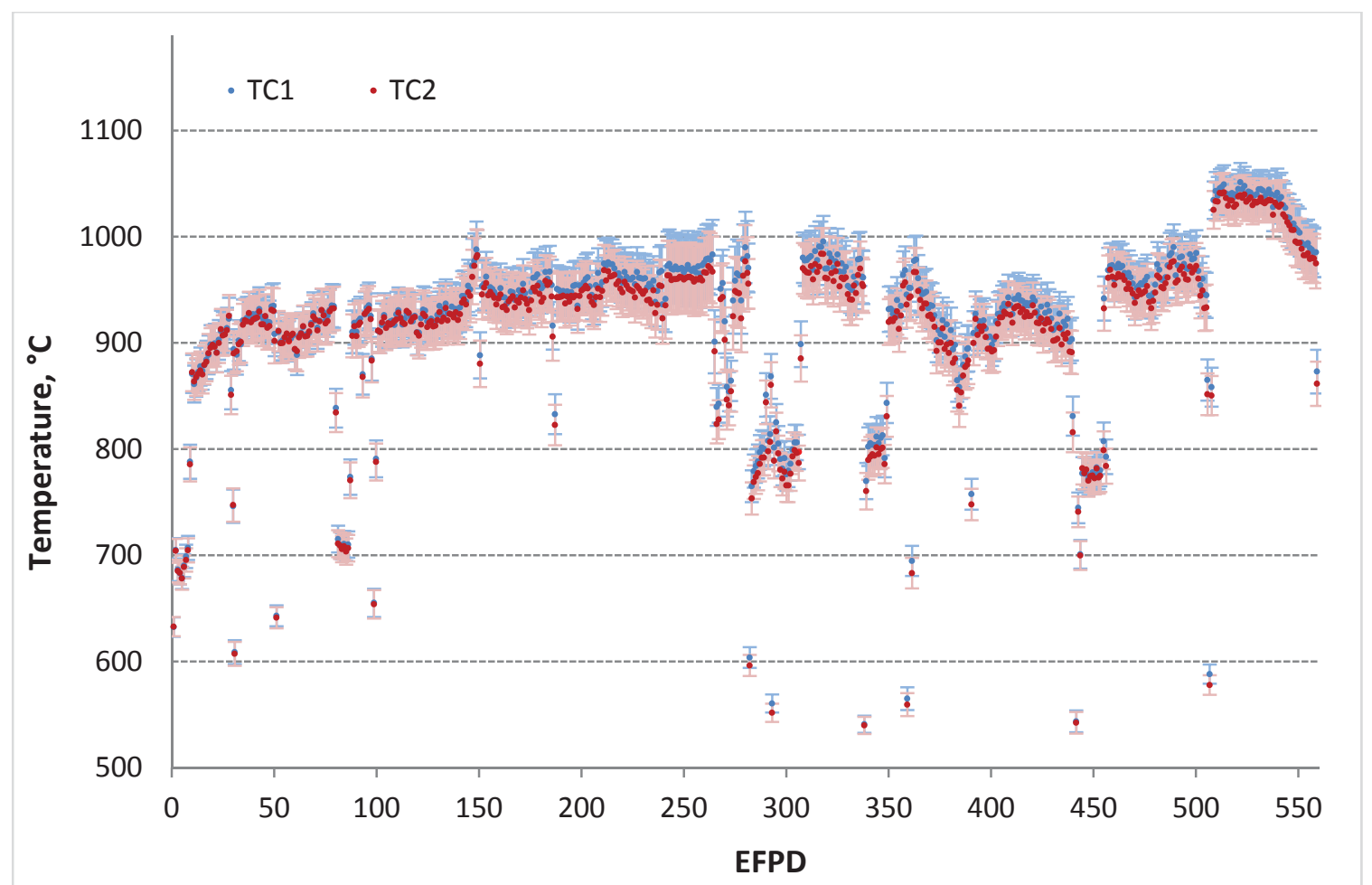

Figure 52. Model temperature and standard deviation of thermocouples in Capsule 3.

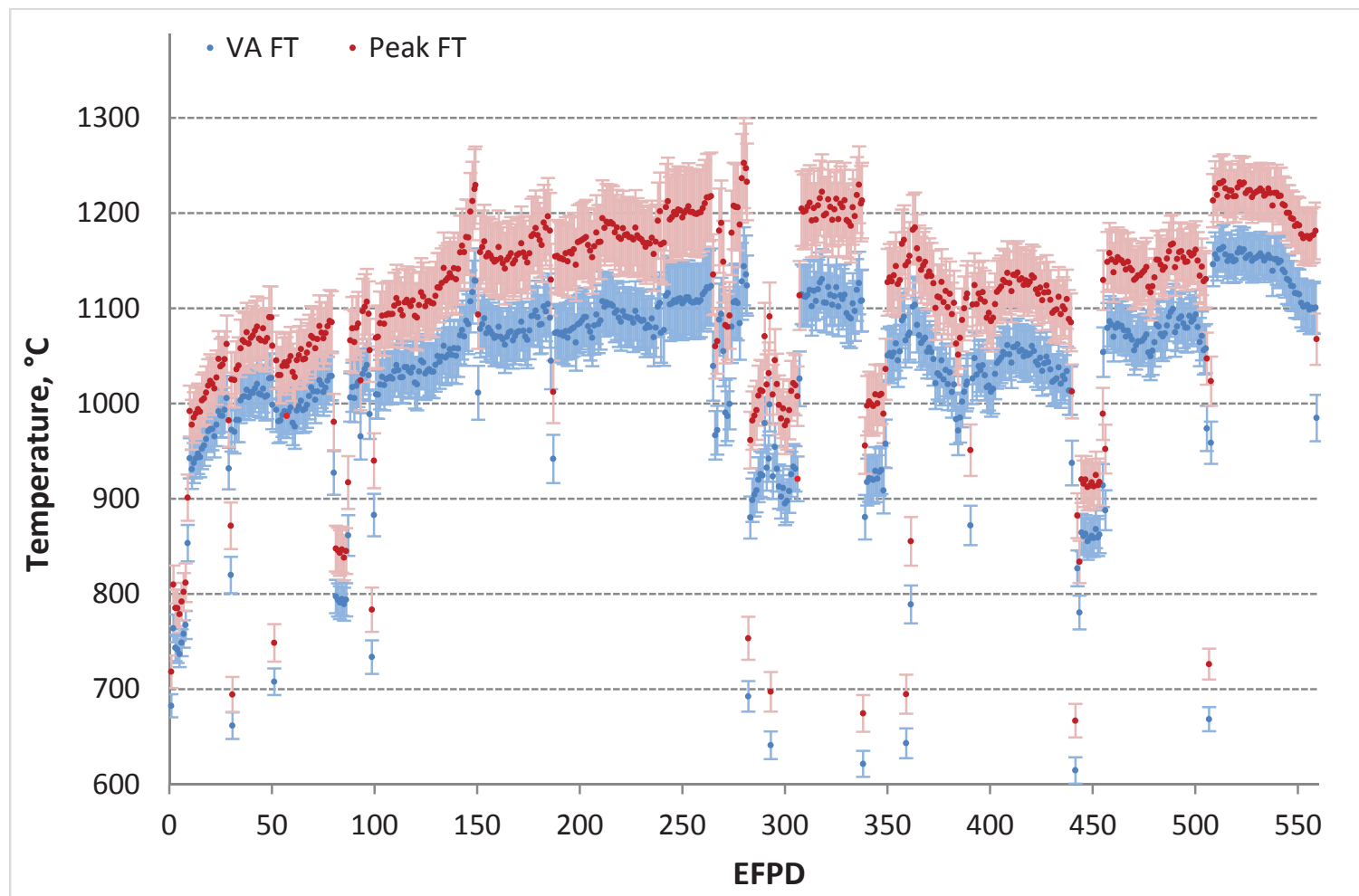

Figure 53. Model temperature and standard deviation of daily averaged fuel temperatures in Capsule 3. 


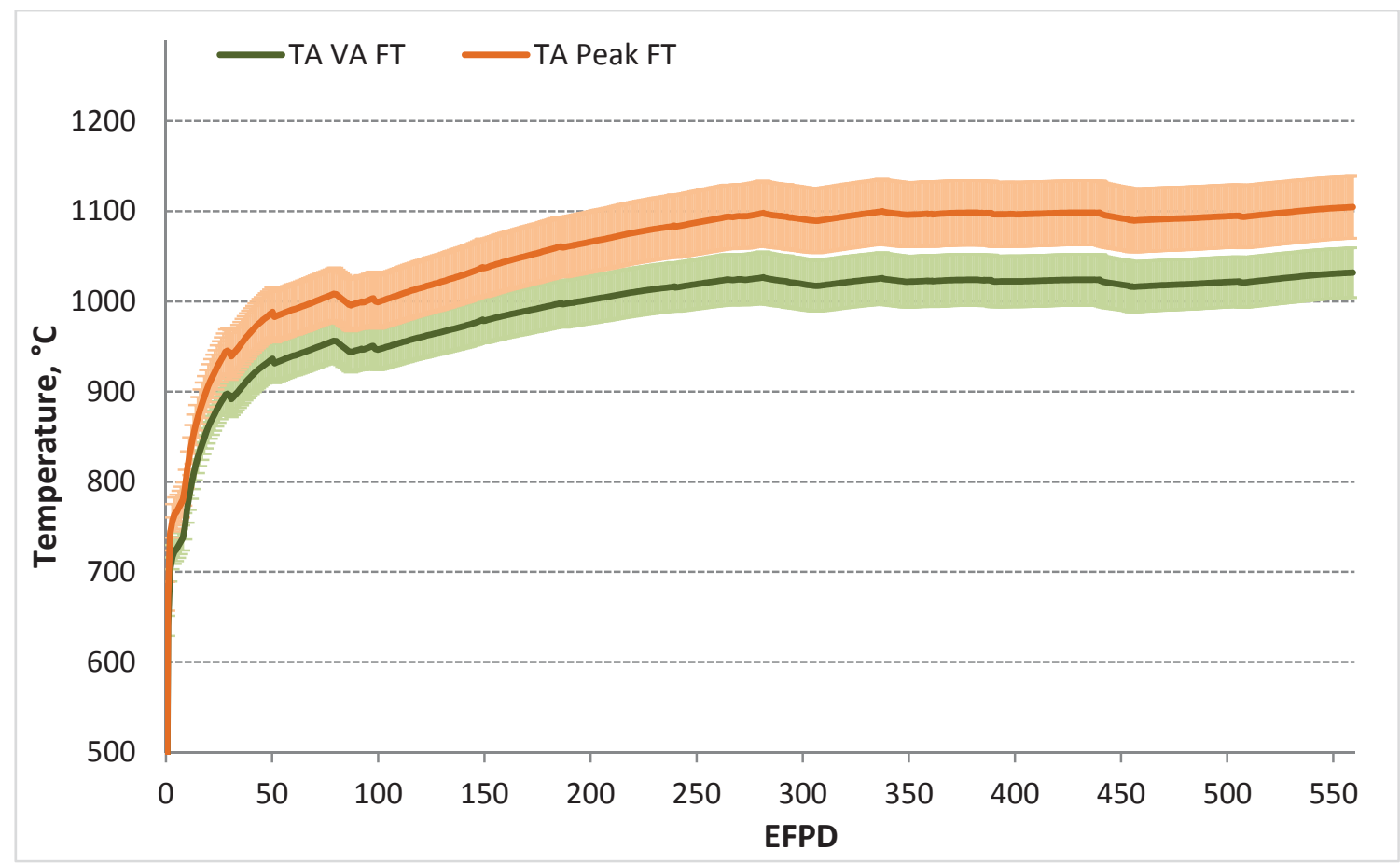

Figure 54. Model temperature and standard deviation of time-average fuel temperatures in Capsule 3.

\subsection{Temperature Uncertainty Results for Capsule 2}

Table 11 summarizes the results for Capsule 2, which is the second from the bottom of the AGR-2 test train. The relatively large control gap distance here also leads to lower gap uncertainty and sensitivity, which helps reduce temperature uncertainty. Temperature uncertainty results specific for Capsule 2 are:

- Input parameter uncertainty (Figure 55): The uncertainties of fuel heat rate and fuel and graphite thermal conductivities are assumed constant over the entire irradiation as in Capsule 6. The relative uncertainties of neon fraction (green triangles) are varying from $0 \%$ to $15 \%$, except for a few high values during powering up and powering down days. Capsule 2 has the middle control gas gap distance, which leads to gap uncertainties (blue line) in the range between 3.7\% and 5.5\%.

- Input parameter sensitivity (Figure 56): The fuel heat rate sensitivities (red dots) are the greatest for all temperatures for most days of irradiation, except for the last three cycles (153A, 154A, and 154B) when neon fraction is the most sensitive because the neon fraction is close to 1 . The sensitivities of fuel thermal conductivity (orange lines) are smallest, especially for temperature at peripheral TC location (top plot). The absolute value of the graphite thermal conductivity sensitivity (purple lines) is higher than the fuel conductivity sensitivity for TC and VA FT temperature, and slightly lower for peak FT. The control gap sensitivity is around 0.2 for all temperatures.

- Weighted temperature variance (Figure 57): The 5\% error in the fuel heat rate is a dominant factor for all TC, VA and peak FT uncertainties. The fuel thermal conductivity is contributing much more variation for peak FT than for VA fuel and TC temperatures.

- Overall calculated temperature uncertainty: The daily relative (in \%) and absolute (in ${ }^{\circ} \mathrm{C}$ ) $\mathrm{TC}, \mathrm{VA}$, and peak FT uncertainties in terms of standard deviation are presented in Figure 58 and Figure 59. The overall uncertainties of peak FT (light-brown triangles) are the highest among calculated temperatures, up to $3.9 \%$ (or $62^{\circ} \mathrm{C}$ ). 
The daily temperature and one standard deviation of the predicted temperatures in Capsule 2 are plotted for peripheral TC1 and TC2 in Figure 60, daily average VA FT and daily peak FT in Figure 61, and TA VA FT and TA peak FT in Figure 62.

Table 11. Summary of temperature uncertainty results for Capsule 2.

\begin{tabular}{|l|c|c|c|c|c|}
\hline & TC & VA FT & Peak FT & $\begin{array}{c}\text { TA VA FT } \\
\text { at EOE }\end{array}$ & $\begin{array}{c}\text { TA Peak } \\
\text { FT at EOE }\end{array}$ \\
\hline Dominant parameter & $\begin{array}{c}\text { Fuel } \\
\text { heat rate }\end{array}$ & $\begin{array}{c}\text { Fuel } \\
\text { heat rate }\end{array}$ & $\begin{array}{c}\text { Fuel } \\
\text { heat rate }\end{array}$ & & \\
\hline Dominant sensitivity & $0.38-0.52$ & $0.42-0.60$ & $0.43-0.57$ & & \\
\hline $\begin{array}{l}\text { Dominant weighted } \\
\text { variance, \% }\end{array}$ & $3.7-7.0$ & $4.6-8.8$ & $4.7-8.2$ & & \\
\hline Relative uncertainty, \% & $2.3-3.6$ & $2.6-3.7$ & $3.0-3.9$ & 3.0 & 3.3 \\
\hline Absolute uncertainty, ${ }^{\circ} \mathrm{C}$ & $26-45$ & $32-54$ & $39-62$ & 37 & 45 \\
\hline
\end{tabular}

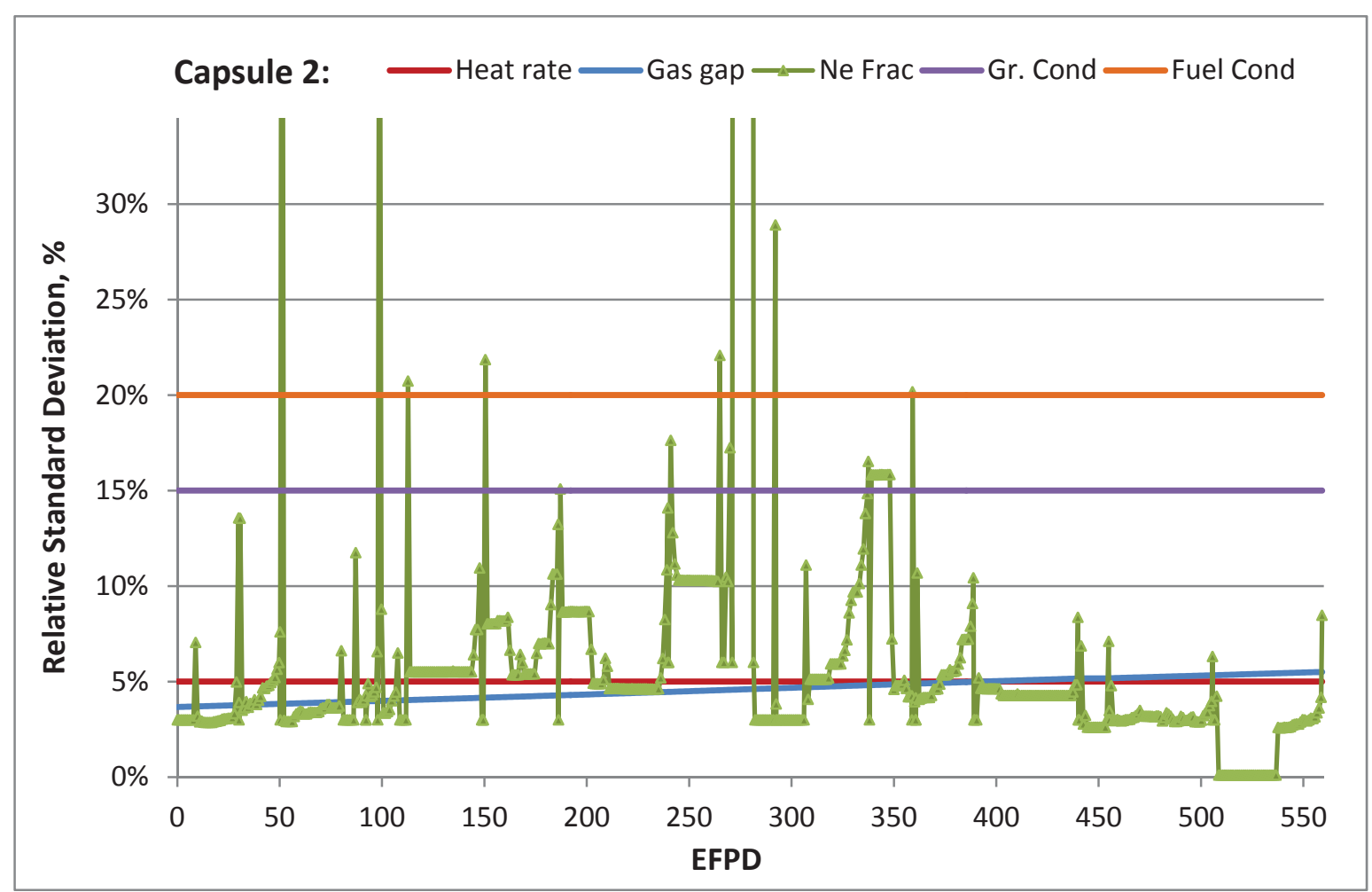

Figure 55. Daily input relative standard deviations for Capsule 2. 


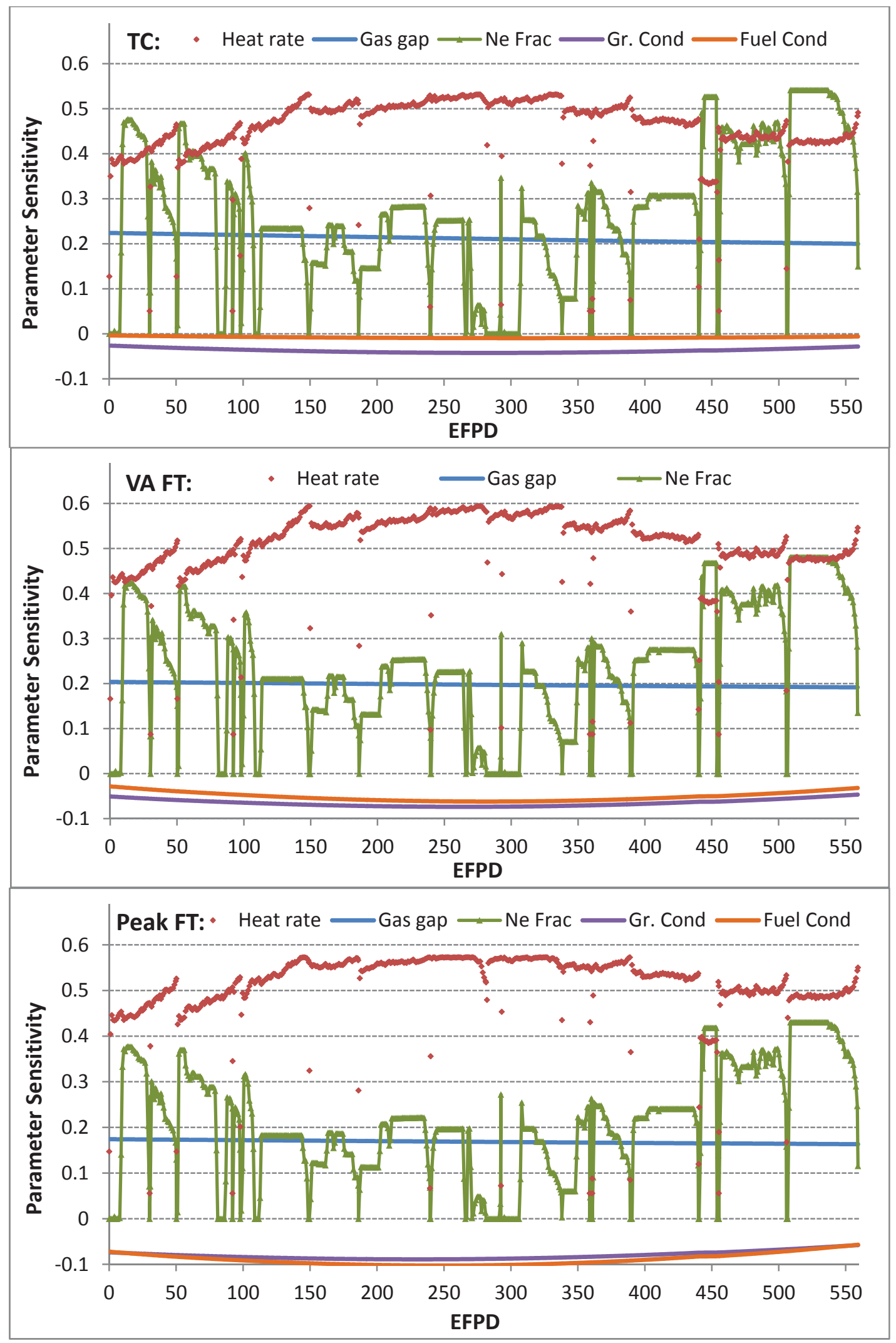

Figure 56. Daily parameter sensitivities for temperatures in Capsule 2. 


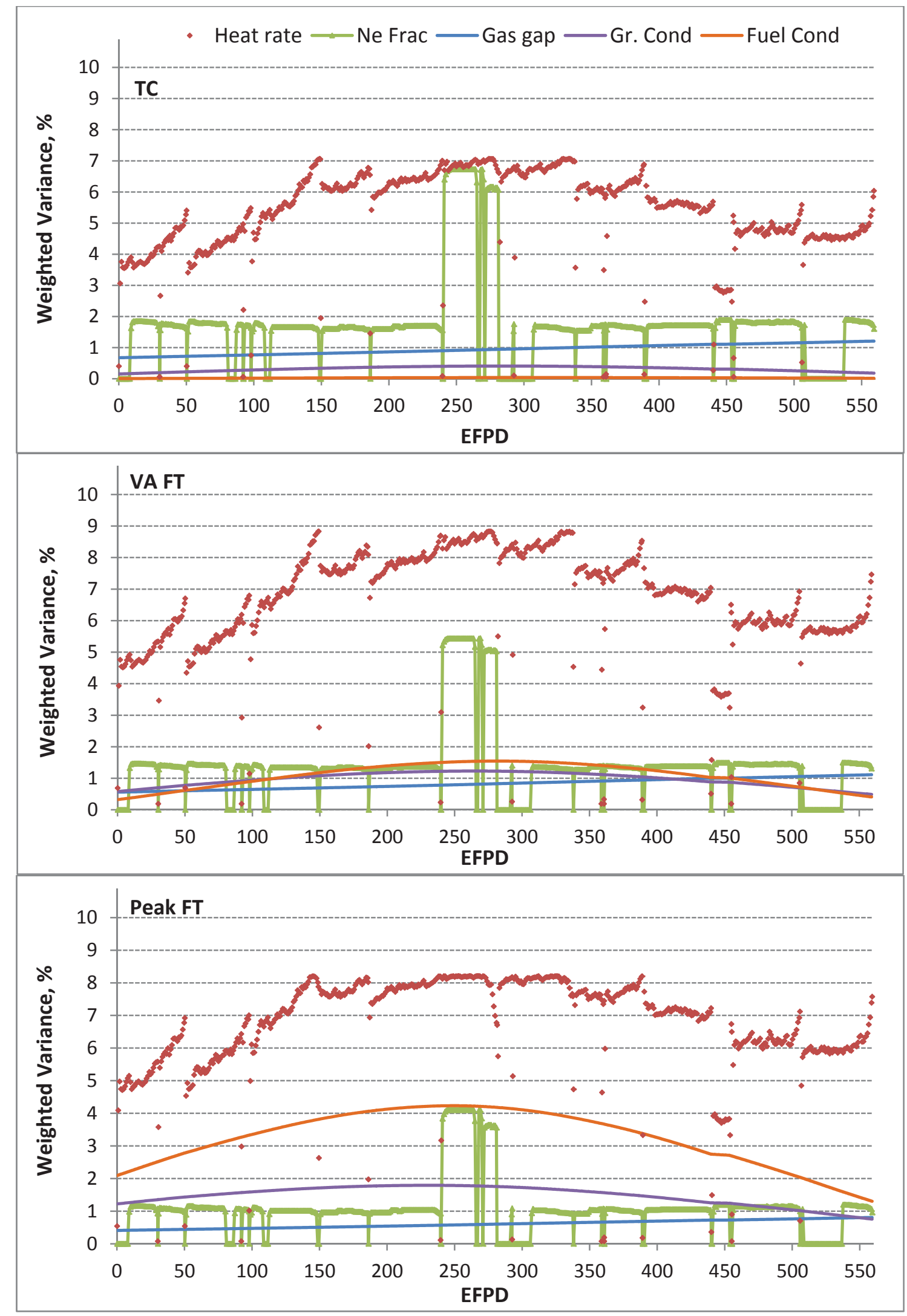

Figure 57. Daily temperature variances due to input uncertainties in Capsule 2. 


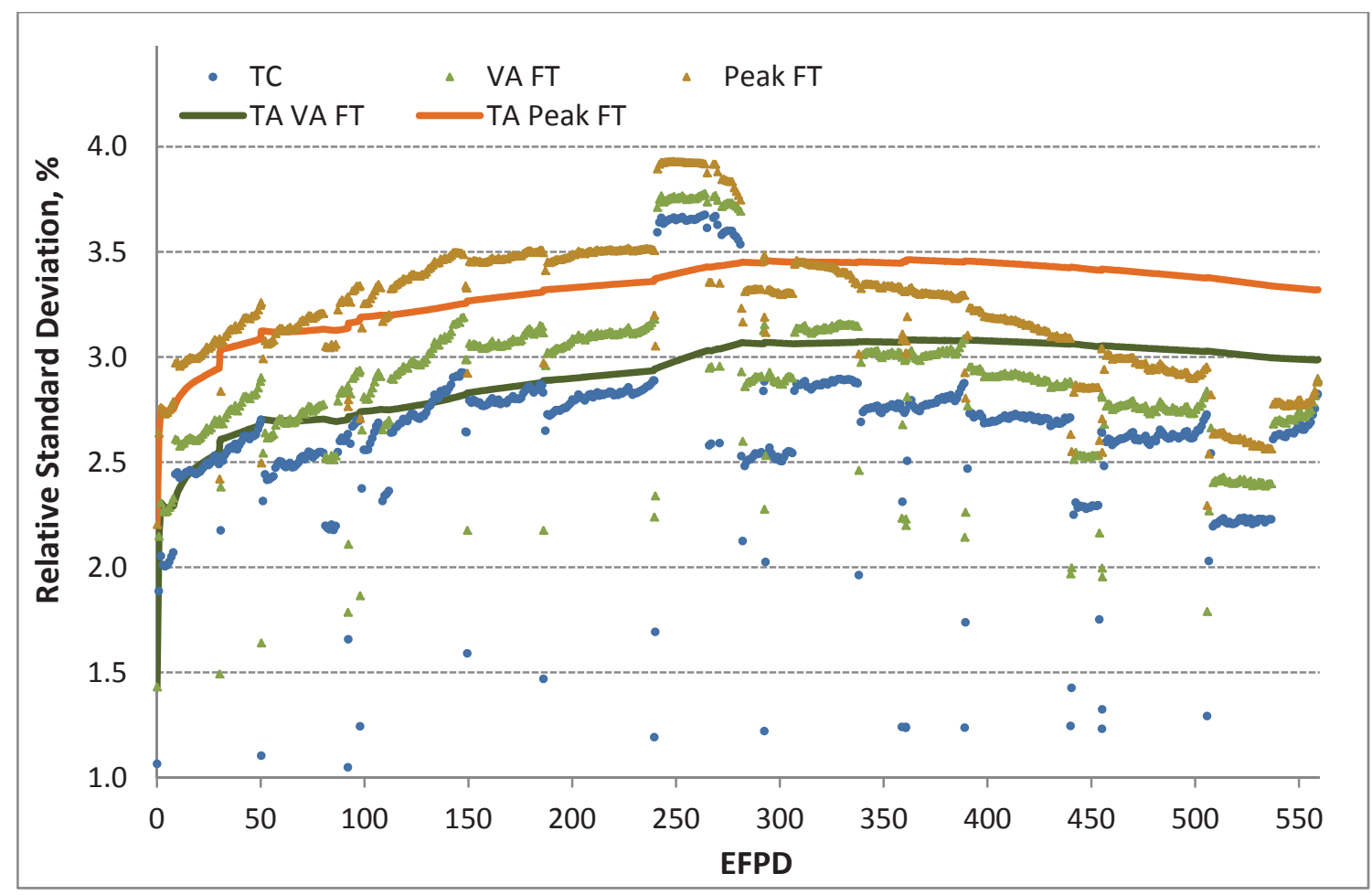

Figure 58. Capsule 2 temperature uncertainties in terms of relative standard deviations.

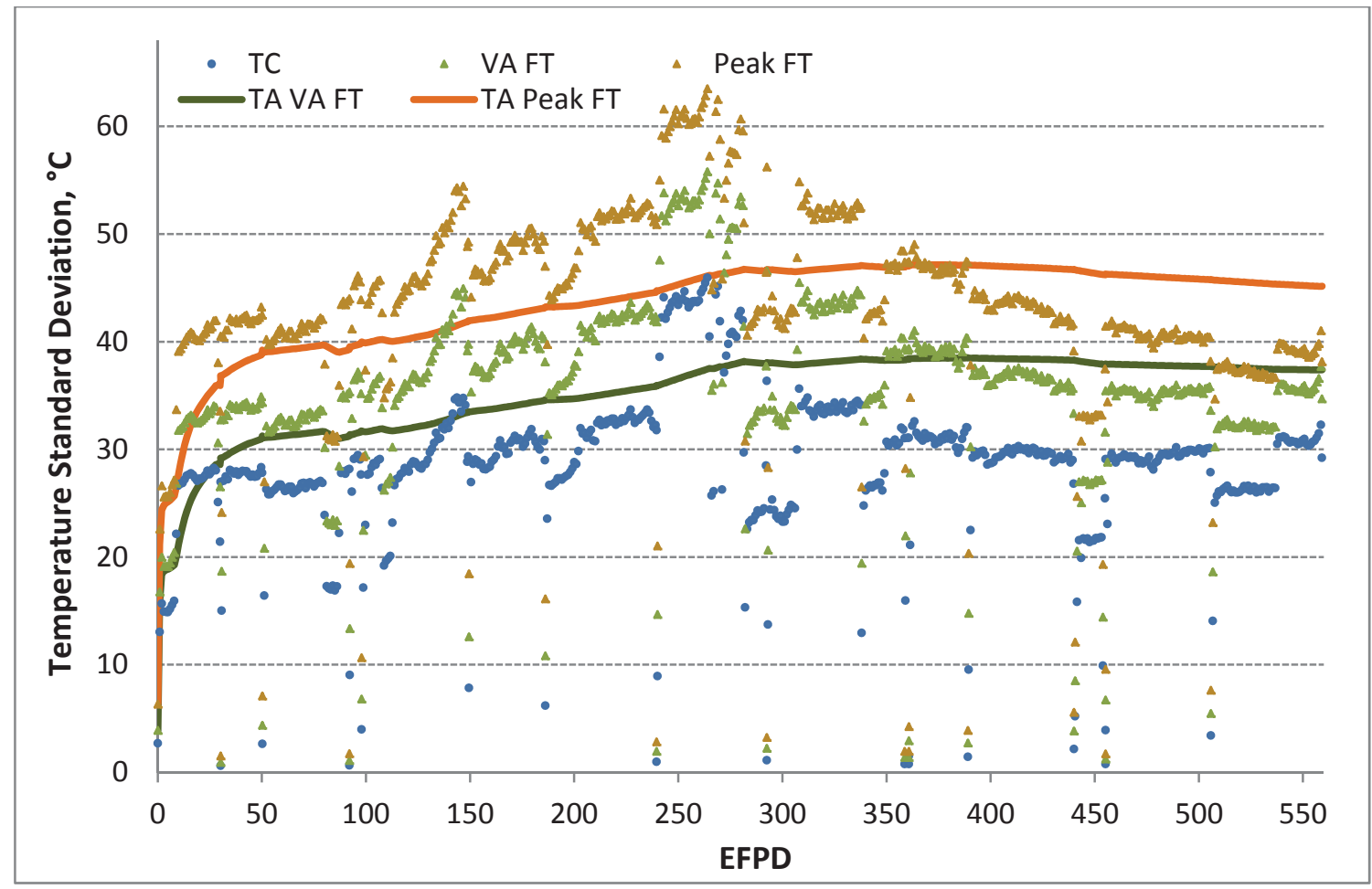

Figure 59. Daily standard deviations of predicted TC and fuel temperatures in Capsule 2. 


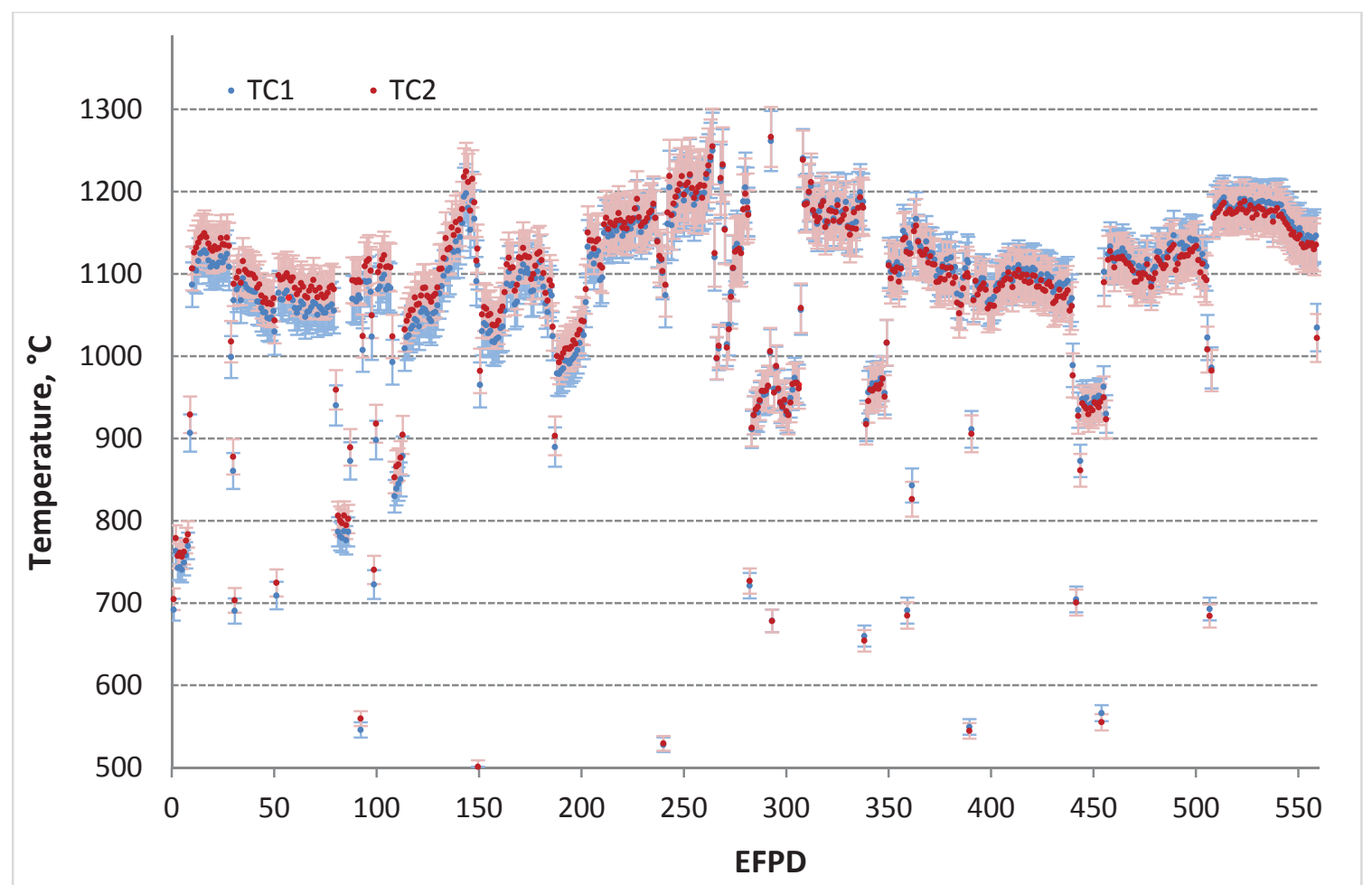

Figure 60. Model temperature and standard deviation of TCs in Capsule 2.

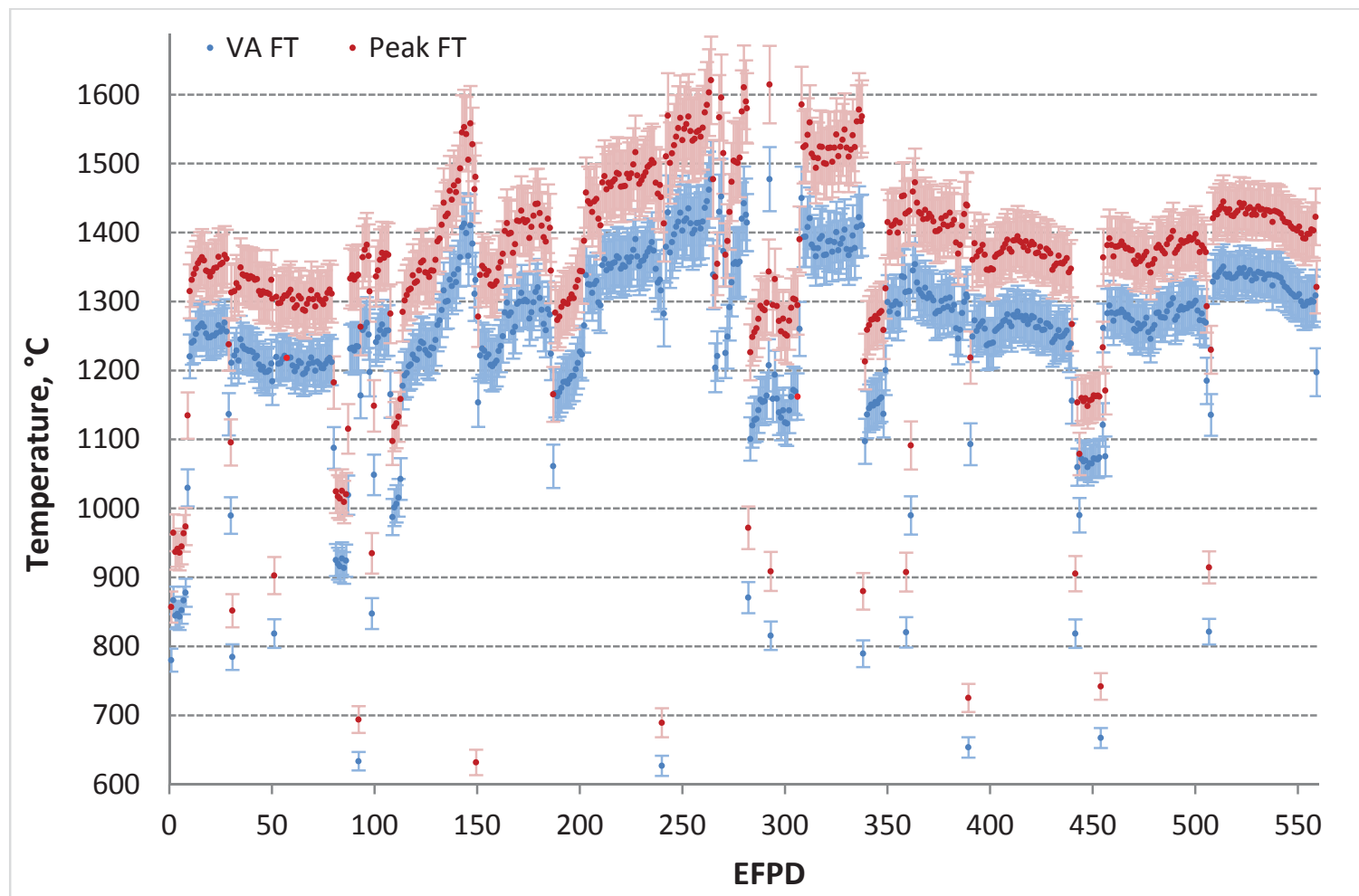

Figure 61. Model temperature and standard deviation of daily averaged fuel temperatures in Capsule 2. 


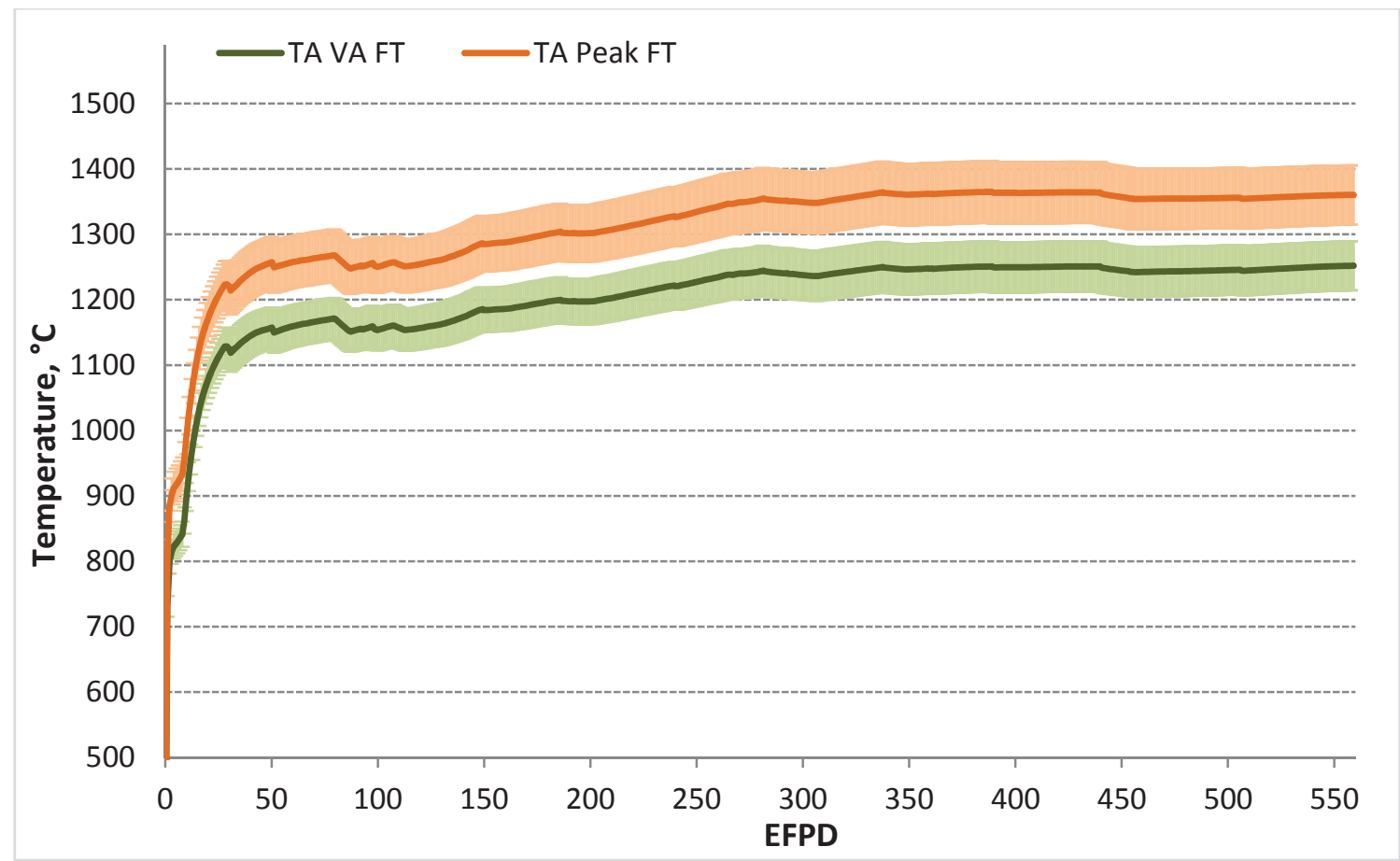

Figure 62. Model temperature and standard deviation of time-average fuel temperatures in Capsule 2. 


\section{CONCLUSIONS}

Knowledge of the thermal conditions and associated uncertainties of the nuclear fuel in a reactor experiment are central to the interpretation of the experiment results. It is necessary when using the experiment results for calibration and validation of nuclear fuel performance models and codes, ultimately in support of the design and licensing of the new nuclear fuel. The work documented in this report supports quantification of uncertainty in the computed thermal condition of nuclear fuels in the AGR-2 test, where it is not practical to obtain direct temperature measurements in the fuel compact domain.

The experiment was instrumented with TCs to provide temperature measurements in the graphite blocks surrounding the fuel compacts. In-depth analysis and qualification of the TC data for the AGR-1 experiment were performed in previous works using statistical methods (Pham and Einerson 2011, 2013). These analyses demonstrate that the AGR-1 TC data can be used to assess and calibrate thermal analysis models in the ABAQUS code (ECAR-2476). Because AGR-1 and AGR-2 capsules are similar, the AGR-2 TC data also can be used to assess and calibrate thermal analysis models in the ABAQUS code (ECAR-2476) for AGR-2 capsules. The calibrated code was then used to predict temperatures in the AGR-2 fuel compact.

This study uses thermal model parameters of potential importance to the AGR-2 predicted FTs based on the combination of input uncertainty and sensitivity. Expert judgment is used as a basis to specify the uncertainty range for a set of select parameters. This also takes into account all events that occurred during AGR-2 irradiation which can impact input uncertainties (e.g., the cross-talk of capsule gas lines occurring from ATR Cycle 150B increased the uncertainty of the neon fraction). Propagation of model parameter uncertainty is then used to quantify the overall uncertainty of AGR-2 calculated temperatures. The model-form uncertainties are usually judged to be dominant. For example, the sensitivity of the thermal simulation to the gap distance suggests that modeling of phenomena that govern gap thermal resistance is expected to matter the most. In order to reduce this source of uncertainty, the boron content was reduced for AGR-2 graphite to minimize the effect of material shrinkage and thermo-mechanical deformation (i.e., causing asymmetrical changes of gap distance).

The parameter sensitivity defines how the predicted temperature would be influenced by changes in an input parameter. The overall uncertainty of the model output increases as the sensitivity coefficient of an input parameter increases. The sensitivity analysis performed in this work went beyond the traditional local sensitivity. Using experimental design, analysis of pairwise interactions of model parameters was performed to establish sufficiency of the first-order (linear) expansion terms in constructing the response surface. To achieve completeness, uncertainty propagation made use of pairwise noise correlations of model parameters. Further, using an interpolation scheme over the input parameter domain, the analysis obtains time-dependent sensitivity over the test campaign's duration. This allows computation of uncertainty for the predicted peak FTs and the predicted graphite temperatures at TC locations over the whole AGR-2 irradiation period.

The sensitivity analysis of the thermal model for AGR-2 Capsule 5 is performed for four days throughout irradiation to determine the sensitivity coefficients of the most influential parameters as functions of the corresponding input. Then, the daily sensitivity is calculated according to the actual thermal condition on that day (e.g., heat rate, fast fluence, neon fraction etc.). The following conclusions are drawn about the parameter sensitivity coefficients for AGR-2 temperature predictions:

- The sensitivity of the fuel heat rate is the highest (up to 0.6) and almost the same for all predicted temperatures of interest (VA fuel, peak fuel, and TC) for all capsules. This sensitivity is slightly increased when the fuel heat rate increases.

- The sensitivity of the neon fraction increases rapidly as the neon fraction increases ranging between 0.1 and 0.5 . It is highest for TC temperature, followed by VA FT, and is lowest for peak FT. 
- The sensitivity of the control gas gap ranged from 0.16 to 0.38 for all calculated temperatures. These sensitivities reached their highest values for temperatures of the peripheral TCs in Capsule 5, which has the smallest gas gap distance. The gap sensitivity is lowest for peak FT because it is usually at the center of a fuel compact far away from the control gap. As the gap distance increases, the gap sensitivity decreases, especially for temperature at peripheral TC location in graphite holder.

- The sensitivity of fuel compact thermal conductivity and graphite holder thermal conductivity are the lowest among selected inputs, ranging from -0.003 to -0.1 . They are near zero for peripheral TC temperature, indicating that the peripheral TC temperatures are insensitive to variations of fuel conductivity and graphite holder conductivity. In contrast, they are much higher for FTs. The sensitivity of fuel conductivity reached the highest absolute value for peak FT near the middle of the irradiation campaign.

The overall uncertainty of calculated temperatures, in terms of one standard deviation, is obtained through propagation of model parameter uncertainty as the square root of the summation of the variances weighted by the squares of their sensitivity coefficients. Thus, the effect of a parameter uncertainty on the variation of temperature prediction is a product of input uncertainty and the sensitivity coefficient. The most significant factors contributing to overall uncertainty of the AGR-2 temperature predictions are:

- For TC temperature, the fuel heat rate is the most influential factor for three capsules $(6,3$, and 2). The higher control gap uncertainty and sensitivity in Capsule 5 due to its smaller gap distance make it the most dominant factor for TC temperature uncertainty. The fuel compact heat rate is the second factor on TC temperature uncertainty. In contrast, the fuel compact and graphite holder conductivities have almost no influence on TC temperature uncertainty.

- For VA and peak FT, the fuel compact heat rate is the most influential factor for all four capsules. The impact is highest near the middle of the irradiation, when the fuel heat rate reached its highest level. The high gas gap uncertainty is the second factor for FT uncertainty in Capsule 5, while the fuel and then the graphite thermal conductivity are the next influential factors in the other three capsules. The fuel thermal conductivity has much larger impact on the uncertainty of peak FT than VA FT.

- It is worth mentioning that the assumed doubled uncertainty of the neon fraction due to the gas line cross-talk failure during ATR Cycle 150B becomes the second most influential factor for all temperatures, even though neon fraction uncertainty has a relatively small impact during other cycles.

The overall uncertainty in the calculated temperatures for AGR-2 ranged from $2.0 \%$ to $6.5 \%$, depending on irradiation time (thermal conditions) capsule and the temperature characteristic being predicted (peak, VA, or TC). Result highlights are:

- For temperatures at TCs, the overall uncertainty ranged from $2.1 \%$ to $4.5 \%$ or $17^{\circ} \mathrm{C}$ to $45^{\circ} \mathrm{C}$. The highest uncertainty of $4.5 \%\left(\sim 45^{\circ} \mathrm{C}\right)$ is for the peripheral TC in Capsule 5 during ATR Cycle $150 \mathrm{~B}$ due to both high gap uncertainty and high neon fraction uncertainty caused by the gas line cross -talk failure.

- For instantaneous FTs, the daily VA temperature uncertainties are lower than peak temperature uncertainties: (i) the uncertainty ranged from $2.2 \%$ to $4.2 \%$ (up to $\sim 54^{\circ} \mathrm{C}$ ) for VA temperature; and (ii) ranged from $2.7 \%$ to $4.2 \%$ (up to $\sim 62^{\circ} \mathrm{C}$ ) for peak temperatures. The FT uncertainty reaches its highest value at the time when the sensitivity of fuel heat rate, fuel and graphite thermal conductivities are highest. The high neon fraction uncertainty during this same time also adds to high overall uncertainty of temperature predictions. 
- The time-average VA FT uncertainty is in the range between $2.7 \%$ and $3.5 \%$ and reaches the highest level of $3.5 \%\left(\sim 39^{\circ} \mathrm{C}\right)$ for Capsule 5 by the end of irradiation. The time-average peak FT uncertainty is in the range between $3.1 \%$ and $3.7 \%$ and reaches the highest level of $3.7 \%\left(\sim 45^{\circ} \mathrm{C}\right)$ also for Capsule 5 by the end of irradiation.

- The overall uncertainties of the AGR-2 calculated temperatures are comparable to those seen in AGR-1. For instantaneous FTs, the relative input uncertainty in AGR-1 ranged from 3\% to 4\% for VA temperatures and ranged from $3 \%$ to $5 \%$ for peak temperatures (up to $\sim 65^{\circ} \mathrm{C}$ ). However, the $10 \%$ bias in the AGR-1 Capsule 6 fuel heat rate increased the overall uncertainty in that capsule to 5.8\%. For time-average FTs at the end of irradiation, the overall uncertainty in AGR-1 was $5.0 \%$ for Capsule 6.

In addition to model-parameter uncertainties analyzed in this study, other epistemic uncertainties exist. In this case, these uncertainties can be categorized into three groups. The first group belongs to biases and errors in expert assessment of the range of uncertainty associated with input parameters. The second group includes modeling assumptions used to build the ABAQUS model for the AGR-2 test. The third group is associated with numerical treatment (e.g., discretization errors) needed to implement and operate the ABAQUS simulations. Although the effect of the first and second groups is generally very hard to evaluate, it is important to systematically delineate them, so not to over-state the confidence in predicted values (underestimating their uncertainties) stemming from a model-parameter uncertainty analysis alone. 


\section{REFERENCES}

Demkowicz, P., Cole, L., Ploger, S., and Winston, P. 2011, AGR-1 Irradaiated Test Train Preliminary Inspection and Disassembly First Look, INL/EXT-10-20722, January 2011.

ECAR-2066, "JMOCUP As-Run Daily Depletion Calculation for the AGR-2 Experiment in ATR B-12 Position," Revision 2, April 2014.

ECAR-2476, “AGR-2 Daily As-Run Thermal Analyses,” Revision 0, INL/MIS-14-31871, August 13, 2014.

Gontard, R. and Nabielek, H., 1990, "Performance Evaluation of Modern HTR TRISO Fuels," Forschungszentrum Julich GmbH., HTA-IB-05/90.

Hawkes, G., Sterbentz, J., and Pham, B., 2014, "Thermal Predictions of The AGR-2 Experiment with Variable Gas Gaps,” INL/CON-14-31073, Paper No. 10218, ANS Summer Meeting, Reno, NV, January 15, 2014.

Hawkes G. L., James Sterbentz, and John Maki, 2011, "Sensitivity Evaluation of the Daily Thermal Predictions of the AGR-1 Experiment in the Advanced Test Reactor," Nice, France: ICAPP 2011 Conference, INL/CON-10-20743, January 7, 2011.

Ostle, B. and Mensing, R., 1975, Statistics in Research, 3rd Edition, Ames, Iowa: Iowa State University Press.

Petti, D., Maki, J., Hawkes, G., Sterbentz, J., Einerson, J., et al., 2014, “ART Program Meeting for AGR-2 Thermal Model Input Uncertainty Estimation,” July 2014.

Pham, B. T. and Einerson, J. J., 2013, "The statistical analysis techniques to support the NGNP fuel performance experiments," Journal of Nuclear Materials, Vol. 441, Issues 1-3, October 2013, pp. 563-573.

Pham, B. T. and Einerson, J.J., 2011, "Simulation-Aided Qualification of Thermocouple Data for AGR Experiments," Transactions-American Nuclear Society, Vol. 104, pp.274-275.

Pham, B. T., Einerson, J. J., and Hawkes, G. L., 2013, Uncertainty Quantification of Calculated Temperatures for the AGR-1 Experiment, Revision 1, INL/EXT-12-25169, March 2013.

Pham, B. T., Hawkes, G. L., and Einerson, J. J., 2014, "Improving Thermal Model Prediction through Statistical Analysis of Irradiation and Post-Irradiation Data from AGR Experiments," Journal of Nuclear Engineering and Design, Vol. 271, May 2014, pp. 209-216.

Pham, B. T. and Jeffrey Einerson, 2014, AGR-2 Final Data Qualification Report for U.S. Capsules ATR Cycles 147A through 154B, INL/EXT-14-32376, July 2014.

PLN-3636, "Technical Program Plan for the Very High Temperature Reactor Technology Development Office/Advanced Gas Reactor Fuel Development and Qualification Program," Revision 3, INL/MIS10-20662, May 2014.

PLN-3798, “AGR-2 Irradiation Experiment Test Plan,” Revision 1, October 2011.

SAS, 2009, JMP 8 Statistics and Graphics Guide, Cary, NC: SAS Institute Inc., Second Edition.

Snead, L.L. and Burchell, T.D, 1995, "Reduction in Thermal Conductivity Due to Neutron Irradiation," 22nd Biennial Conference on Carbon, pp. 774-775.

SPC-1064, “AGR-2 Irradiation Test Specification,” Revision 1, June 2010.

TEV-2004, “AGR-2 Neon Fraction Estimation,” Revision 0, February 2014. 